\title{
[3 + 2]-Cycloaddition reaction of sydnones with alkynes
}

\author{
Veronika Hladíková, Jiří Váňa and Jiří Hanusek ${ }^{*}$
}

\author{
Review \\ Address: \\ Institute of Organic Chemistry and Technology, University of \\ Pardubice, Studentská 573, 53210 Pardubice, Czech Republic \\ Email: \\ Jiří Hanusek* - Jiri.Hanusek@upce.cz \\ * Corresponding author \\ Keywords: \\ alkynes; $\mathrm{Cu}(\mathrm{I})$ catalysis; [3 + 2]-cycloaddition; mechanism; \\ regioselectivity; sydnones
}

\author{
Beilstein J. Org. Chem. 2018, 14, 1317-1348. \\ doi:10.3762/bjoc. 14.113 \\ Received: 20 February 2018 \\ Accepted: 11 May 2018 \\ Published: 05 June 2018 \\ Associate Editor: I. R. Baxendale \\ (C) 2018 Hladíková et al.; licensee Beilstein-Institut. \\ License and terms: see end of document.
}

\begin{abstract}
This review covers all known examples of [3+2]-cycloaddition between sydnones and both terminal as well as internal alkynes/ cycloalkynes taken from literature since its discovery by Huisgen in 1962 up to the current date. Except enumeration of synthetic applications it also covers mechanistic studies, catalysis, effects of substituents and reaction conditions influencing reaction rate and regioselectivity.
\end{abstract}

\section{Review \\ Introduction}

Since Huisgen's discovery of the $[3+2]$-cycloaddition between 3 -substituted sydnones and both terminal as well as internal alkynes $[1,2]$ many researchers have tried to utilize this synthetic approach for the synthesis of polysubstituted 1,2diazoles (pyrazoles, indazoles). However, until 2013 when Taran's group introduced the regioselective $\mathrm{Cu}(\mathrm{I})$-phenanthroline catalysis [3] this method was of limited value due to the harsh reaction conditions and sometimes also due to low regioselectivity in those cases when a non-symmetrical alkyne was employed as a reactant. Surprisingly, until the fall of 2017, no comprehensive work concerning this important topic was published. This encouraged us to write this review. During its completion a new feature article bridging this gap was published by Taran et al. [4]. In order to avoid duplication our review is therefore focused in more detail on thermal, photo- chemical as well as metal-catalyzed reactions of sydnones with alkynes and factors that influence the yield and ratio of both possible regioisomers and also the kinetics and mechanism of this cycloaddition reaction.

\section{Thermal reaction of sydnones with symmetrical alkynes and cycloalkynes}

As mentioned above, the thermal reaction of 3-alkyl-, 3-aryl- or even 3-substituted aminosydnones with symmetrical alkynes (Scheme 1) represents a very useful and straightforward method for the synthesis of substituted 1,3,4-tri- or 1,3,4,5-tetrasubstituted pyrazoles [1,2,5-39] or indazoles.

Dimethyl acetylenedicarboxylate (DMAD, $\left.\mathrm{R}^{3}=\mathrm{COOMe}\right)$ or its analogues (diethyl; $\mathrm{R}^{3}=\mathrm{COOEt}$ ); di-tert-butyl, $\mathrm{R}^{3}=\mathrm{COO} t$-Bu 


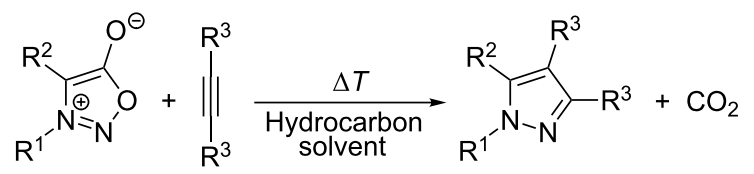

$\mathrm{R}^{1}: \mathrm{Me}, \mathrm{Bn}, \mathrm{Ar}, \mathrm{NMe}_{2}, \mathrm{~N}\left(\mathrm{CH}_{2} \mathrm{CH}_{2}\right)_{2} \mathrm{O}, \mathrm{N}\left(\mathrm{CH}_{2}\right)_{5}$ $\mathrm{R}^{2}$ : Me, $\mathrm{Ph}$, halogen, MeS, PhS, MeCO, PhCO, COOMe... $\mathrm{R}^{3}: \mathrm{H}, \mathrm{Ph}, \mathrm{PhCO}, \mathrm{COOMe}(\mathrm{Et})$

Scheme 1: Thermal reaction of sydnones with symmetrical alkynes.

etc.) act as the most common dipolarophiles because of their high reactivity. Moreover, one or both carboxylate groups in position 3 and 4 in the final pyrazole are easily removable using a hydrolysis/decarboxylation protocol $[5,16]$ thus giving pyrazole-4-carboxylic acids - potent xanthine oxidoreductase inhibitors [40] or even 3,4-unsubstituted pyrazoles [16]. Both pyrazole carboxylic groups can be also modified to hydrazides and oxazole rings [26] or a new condensed pyridazine ring [27] Less reactive dipolarophiles such as dibenzoylacetylenes $(1,4-$ diphenylbut-2-yn-1,4-diones) [13,17,38,39], diphenylacetylene
$[1,2,9,13,15,32]$ or even acetylene itself $[1,2]$ have also been successfully reacted with sydnones. The most typical procedure involves heating both components in boiling hydrocarbon solvent (benzene, toluene or xylene) for several hours (up to $24 \mathrm{~h}$ ) and the isolated yields are often close to $90 \%$ for the ordinary substituents (alkyls, aryls, halogens) of the sydnone. Somewhat lower yields were obtained in ethyleneglycol [5]. The reaction of the parent 1-phenylsydnone with DMAD and its diethyl analogue has also been performed in supercritical carbon dioxide [41] in which 65 and $83 \%$ yields of dimethyl (or diethyl) 1-phenylpyrazole-3,4-dicarboxylates were achieved. Only in two cases involving 3-(2,4,6-trisubstituted phenyl)-4-iodosydnones ( $\mathrm{R}^{1}:$ 2-Br-4,6-diMe-Ph, 2,4-diBr-6-Me-Ph; $\left.\mathrm{R}^{2}: \mathrm{I}\right)$ and DMAD (or its diethyl analogue) did the cycloaddition completely fail $[21,22]$ even after heating for 3 days in boiling xylene. This result was explained by the steric hindrance between the bulky substituents in the 4-position (iodine) and the substituents $(\mathrm{Me}, \mathrm{Br})$ in both ortho-positions of the adjacent 2,4,6-trisubstituted phenyl ring. All the examples found for $[3+2]$-cycloadditions between sydnones and symmetrical noncyclic alkynes including conditions used for the synthesis, are presented in Table 1.

Table 1: Thermal cycloaddition of sydnones with symmetrical non-cyclic alkynes.

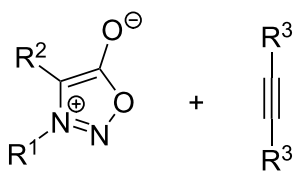

conditions<smiles>[R]c1nn([R])c([R])c1[R]</smiles>

\begin{tabular}{|c|c|c|c|c|c|c|}
\hline entry & $\mathrm{R}^{1}$ & $\mathrm{R}^{2}$ & $\mathrm{R}^{3}$ & conditions & yield [\%] & ref. \\
\hline 1 & $\mathrm{Ph}$ & $\mathrm{H}$ & $\mathrm{H}$ & acetone, $170^{\circ} \mathrm{C}, 25 \mathrm{~h}$ & 75 & {$[1,2]$} \\
\hline 2 & $\mathrm{Ph}$ & $\mathrm{Me}$ & $\mathrm{Ph}$ & $180^{\circ} \mathrm{C}, 5 \mathrm{~h}$ & $96-97$ & {$[1,2]$} \\
\hline 3 & $\mathrm{Ph}$ & $\mathrm{H}$ & $\mathrm{Ph}$ & $160^{\circ} \mathrm{C}, 4.5 \mathrm{~h}$ & 93 & [2] \\
\hline 4 & $\mathrm{Ph}$ & $\mathrm{Ph}$ & $\mathrm{Ph}$ & $190^{\circ} \mathrm{C}, 9 \mathrm{~h}$ & 98 & [2] \\
\hline 5 & $\mathrm{Ph}$ & $\mathrm{H}$ & COOMe & $\begin{array}{l}\text { toluene, } 90^{\circ} \mathrm{C}, 4 \mathrm{~h} \\
\text { xylene, reflux } \\
p \text {-xylene, reflux, overnight } \\
p \text {-xylene, reflux, overnight } \\
p \text {-xylene, reflux, } 4 \mathrm{~h}\end{array}$ & $\begin{array}{l}92 \\
92 \\
98 \\
93 \\
93\end{array}$ & $\begin{array}{l}{[1,2]} \\
{[20]} \\
{[26]} \\
{[29]} \\
{[31]}\end{array}$ \\
\hline 6 & $\mathrm{Ph}$ & $\mathrm{Me}$ & coOMe & xylene, $120^{\circ} \mathrm{C}, 1 \mathrm{~h}$ & 99 & {$[1,2]$} \\
\hline 7 & $\mathrm{Bn}$ & $\mathrm{H}$ & coOMe & xylene, $120^{\circ} \mathrm{C}, 5 \mathrm{~h}$ & $93-98$ & {$[1,2]$} \\
\hline 8 & $\mathrm{Ph}$ & $\mathrm{Cl}$ & coome & $\begin{array}{l}\text { ethyleneglycol, } 120^{\circ} \mathrm{C}, 1 \mathrm{~h} \\
\text { xylene, reflux }\end{array}$ & $\begin{array}{l}74 \\
60-80\end{array}$ & $\begin{array}{l}{[5]} \\
{[6]}\end{array}$ \\
\hline 9 & $\mathrm{Ph}$ & $\mathrm{Br}$ & coOMe & $\begin{array}{l}\text { ethyleneglycol, } 120^{\circ} \mathrm{C}, 1 \mathrm{~h} \\
\text { xylene, reflux }\end{array}$ & $\begin{array}{l}70 \\
60-80\end{array}$ & $\begin{array}{l}{[5]} \\
{[6]}\end{array}$ \\
\hline 10 & $\mathrm{Me}$ & $\mathrm{Cl}$ & COOMe & ethyleneglycol, $120^{\circ} \mathrm{C}, 1.5 \mathrm{~h}$ & 12 & {$[5]$} \\
\hline 11 & $\mathrm{Me}$ & $\mathrm{Br}$ & COOMe & ethyleneglycol, $120^{\circ} \mathrm{C}, 1.5 \mathrm{~h}$ & 82 & {$[5]$} \\
\hline 12 & $\mathrm{Ph}$ & $\mathrm{NO}_{2}$ & coOMe & xylene, reflux & $60-80$ & {$[6]$} \\
\hline 13 & 4-Br-Ph & $\mathrm{H}$ & coome & $\begin{array}{l}\text { xylene, reflux } \\
p \text {-xylene, reflux, } 6 \mathrm{~h}\end{array}$ & $\begin{array}{l}60-80 \\
92\end{array}$ & $\begin{array}{l}{[6]} \\
{[35]}\end{array}$ \\
\hline 14 & $4-\mathrm{Br}-\mathrm{Ph}$ & $\mathrm{Br}$ & coOMe & xylene, reflux & $60-80$ & [6] \\
\hline 15 & 4-Br-Ph & $\mathrm{Cl}$ & coOMe & xylene, reflux & $60-80$ & {$[6]$} \\
\hline
\end{tabular}


Table 1: Thermal cycloaddition of sydnones with symmetrical non-cyclic alkynes. (continued)

\begin{tabular}{|c|c|c|c|c|c|c|}
\hline 16 & $4-\mathrm{Cl}-\mathrm{Ph}$ & $\mathrm{H}$ & COOMe & $\begin{array}{l}\text { xylene, reflux } \\
p \text {-xylene, reflux, overnight }\end{array}$ & $\begin{array}{l}60-80 \\
98\end{array}$ & $\begin{array}{l}{[6]} \\
{[26]}\end{array}$ \\
\hline 17 & $4-\mathrm{Cl}-\mathrm{Ph}$ & $\mathrm{Br}$ & coOMe & xylene, reflux & $60-80$ & [6] \\
\hline 18 & 4-Cl-Ph & $\mathrm{Cl}$ & coOMe & xylene, reflux & $60-80$ & [6] \\
\hline 19 & 4-MeO-Ph & $\mathrm{H}$ & COOMe & $\begin{array}{l}\text { xylene, reflux } \\
\text { xylene, reflux, overnight } \\
p \text {-xylene, reflux, } 4 \mathrm{~h}\end{array}$ & $\begin{array}{l}60-80 \\
91 \\
91\end{array}$ & $\begin{array}{l}{[6]} \\
{[29]} \\
{[31]}\end{array}$ \\
\hline 20 & 4-MeO-Ph & $\mathrm{Br}$ & COOMe & xylene, reflux & $60-80$ & [6] \\
\hline 21 & 4-MeO-Ph & $\mathrm{Cl}$ & coOMe & xylene, reflux & $60-80$ & [6] \\
\hline 22 & 4-Br-3-Cl-Ph & $\mathrm{H}$ & coOMe & xylene, reflux & 89 & [7] \\
\hline 23 & 4-Br-3-Cl-Ph & $\mathrm{Br}$ & coOMe & xylene, reflux & 71 & [7] \\
\hline 24 & 4-Br-3-Cl-Ph & $\mathrm{Cl}$ & COOMe & xylene, reflux & 61 & [7] \\
\hline 25 & $4-\mathrm{NO}_{2}-\mathrm{Ph}$ & $\mathrm{H}$ & COOMe & $\begin{array}{l}\text { toluene, } 110^{\circ} \mathrm{C}, 1.75 \mathrm{~h} \\
p \text {-xylene, reflux, overnight }\end{array}$ & $\begin{array}{l}99 \\
98\end{array}$ & $\begin{array}{l}{[8]} \\
{[26]}\end{array}$ \\
\hline 26 & $4-\mathrm{NO}_{2}-\mathrm{Ph}$ & $\mathrm{Ph}$ & COOMe & toluene, $100-105^{\circ} \mathrm{C}, 16 \mathrm{~h}$ & 96 & [8] \\
\hline 27 & 2,4-di-NO $2-\mathrm{Ph}$ & $\mathrm{Ph}$ & COOMe & toluene, $100-105^{\circ} \mathrm{C}, 4 \mathrm{~h}$ & 97 & [8] \\
\hline 28 & $\mathrm{Ph}$ & MeS & COOMe & toluene, $100^{\circ} \mathrm{C}, 2 \mathrm{~h}$ & 96 & [8] \\
\hline 29 & $4-\mathrm{Me}_{2} \mathrm{~N}-\mathrm{Ph}$ & MeS & COOMe & mesitylene, $130-135^{\circ} \mathrm{C}, 0.5 \mathrm{~h}$ & 92 & [8] \\
\hline 30 & $\mathrm{Ph}$ & $\mathrm{PhS}$ & coOMe & xylene, $120-125^{\circ} \mathrm{C}, 5.75 \mathrm{~h}$ & 91 & [8] \\
\hline 31 & $\mathrm{Ph}$ & $\mathrm{PhS}=\mathrm{O}$ & coOMe & mesitylene, $135-140^{\circ} \mathrm{C}, 26 \mathrm{~h}$ & 63 & [8] \\
\hline 32 & $\mathrm{Ph}$ & $\mathrm{MeC}=\mathrm{O}$ & COOMe & xylene, $160^{\circ} \mathrm{C}, 18 \mathrm{~h}$ & 62 & [8] \\
\hline 33 & 4-MeO-Ph & $\mathrm{MeC}=\mathrm{O}$ & coOMe & mesitylene, $160-165^{\circ} \mathrm{C}, 22 \mathrm{~h}$ & 95 & [8] \\
\hline 34 & 4-MeO-Ph & $\mathrm{CN}$ & COOMe & xylene, $160^{\circ} \mathrm{C}, 24 \mathrm{~h}$ & 79 & [8] \\
\hline 35 & $\mathrm{Me}_{2} \mathrm{~N}$ & MeS & COOMe & $\begin{array}{l}\text { xylene, } 160^{\circ} \mathrm{C}, 18 \mathrm{~h} \\
\text { benzene, } 80^{\circ} \mathrm{C}, 16 \mathrm{~h}\end{array}$ & $\begin{array}{l}31 \\
19\end{array}$ & [9] \\
\hline 36 & $\mathrm{Me}_{2} \mathrm{~N}$ & MeS & $\mathrm{Ph}$ & xylene, $155-160^{\circ} \mathrm{C}, 93 \mathrm{~h}$ & 71 & [9] \\
\hline 37 & $\mathrm{Me}_{2} \mathrm{~N}$ & PhS & COOMe & $\begin{array}{l}\text { xylene, } 155-160^{\circ} \mathrm{C}, 19 \mathrm{~h} \\
\text { benzene, } 80^{\circ} \mathrm{C}, 23 \mathrm{~h}\end{array}$ & $\begin{array}{l}30 \\
0\end{array}$ & [9] \\
\hline 38 & $\mathrm{Me}_{2} \mathrm{~N}$ & $\mathrm{H}$ & coOMe & $\begin{array}{l}\text { xylene, } 155-160^{\circ} \mathrm{C}, 3 \mathrm{~h} \\
\text { benzene, } 80^{\circ} \mathrm{C}, 19 \mathrm{~h}\end{array}$ & $\begin{array}{l}9 \\
2\end{array}$ & [9] \\
\hline 39 & $\mathrm{Me}_{2} \mathrm{~N}$ & $\mathrm{CN}$ & COOMe & xylene, $155-160^{\circ} \mathrm{C}, 3 \mathrm{~h}$ & 0 & [9] \\
\hline 40 & $\mathrm{O}\left(\mathrm{CH}_{2} \mathrm{CH}_{2}\right)_{2} \mathrm{~N}$ & MeS & coome & benzene, $80^{\circ} \mathrm{C}, 23 \mathrm{~h}$ & 53 & [9] \\
\hline 41 & $\mathrm{O}\left(\mathrm{CH}_{2} \mathrm{CH}_{2}\right)_{2} \mathrm{~N}$ & $\mathrm{PhS}$ & coOMe & xylene, $155-160^{\circ} \mathrm{C}, 22 \mathrm{~h}$ & 70 & [9] \\
\hline 42 & $\left(\mathrm{CH}_{2}\right)_{5} \mathrm{~N}$ & MeS & COOMe & xylene, $160^{\circ} \mathrm{C}, 20 \mathrm{~h}$ & 47 & [9] \\
\hline 43 & $\left(\mathrm{CH}_{2}\right)_{5} \mathrm{~N}$ & $\mathrm{PhS}$ & coOMe & xylene, $150-160^{\circ} \mathrm{C}, 24 \mathrm{~h}$ & 27 & [9] \\
\hline 44 & & & COOMe & benzene, reflux & 71 & [10] \\
\hline 45 & & & COOMe & benzene, reflux & 77 & [10] \\
\hline 46 & 4-MeCO-Ph & $\mathrm{H}$ & coOMe & xylene, reflux & 56 & [11] \\
\hline 47 & 4-MeCO-Ph & $\mathrm{Me}$ & COOMe & xylene, reflux & 51 & [11] \\
\hline 48 & 4-MeCO-Ph & $\mathrm{Ph}$ & coOMe & xylene, reflux & 38 & [11] \\
\hline 49 & 4-(Me(Ph)NSO$\left.{ }_{2}\right)-\mathrm{Ph}$ & $\mathrm{H}$ & coOMe & xylene, reflux, $2 \mathrm{~h}$ & 75 & [12] \\
\hline 50 & 4-(Et(Ph) $\left.\mathrm{NSO}_{2}\right)-\mathrm{Ph}$ & $\mathrm{H}$ & coOMe & xylene, reflux, $2 \mathrm{~h}$ & 75 & [12] \\
\hline 51 & 4- $\left(\mathrm{O}\left(\mathrm{CH}_{2} \mathrm{CH}_{2}\right)_{2} \mathrm{NSO}_{2}\right)-\mathrm{Ph}$ & $\mathrm{H}$ & coOMe & xylene, reflux, $2 \mathrm{~h}$ & 78 & [12] \\
\hline 52 & $4-\left(\left(\mathrm{CH}_{2}\right)_{5} \mathrm{NSO}_{2}\right)-\mathrm{Ph}$ & $\mathrm{H}$ & coOMe & xylene, reflux, $2 \mathrm{~h}$ & 76 & [12] \\
\hline 53 & $4-\left(\left(\mathrm{CH}_{2}\right)_{4} \mathrm{NSO}_{2}\right)-\mathrm{Ph}$ & $\mathrm{H}$ & coOMe & xylene, reflux, $2 \mathrm{~h}$ & 75 & [12] \\
\hline 54 & $4-\left(\mathrm{Et}_{2} \mathrm{NSO}_{2}\right)-\mathrm{Ph}$ & $\mathrm{H}$ & coOMe & xylene, reflux, $2 \mathrm{~h}$ & 75 & [12] \\
\hline 55 & 4- $\left(\mathrm{O}\left(\mathrm{CH}_{2} \mathrm{CH}_{2}\right)_{2} \mathrm{NSO}_{2}\right)-\mathrm{Ph}$ & $\mathrm{Br}$ & COOMe & xylene, reflux, $2 \mathrm{~h}$ & 66 & [12] \\
\hline 56 & $4-\left(\left(\mathrm{CH}_{2}\right)_{5} \mathrm{NSO}_{2}\right)-\mathrm{Ph}$ & $\mathrm{Br}$ & COOMe & xylene, reflux, 2 h & 70 & [12] \\
\hline 57 & $\mathrm{CH}_{2} \mathrm{CH}$ & & $\mathrm{Ph}$ & xylene, reflux, $48 \mathrm{~h}$ & 45 & [13] \\
\hline 58 & $\mathrm{CH}_{2} \mathrm{CH}$ & & coOMe & xylene, reflux, 8 h & 80 & [13] \\
\hline
\end{tabular}


Table 1: Thermal cycloaddition of sydnones with symmetrical non-cyclic alkynes. (continued)

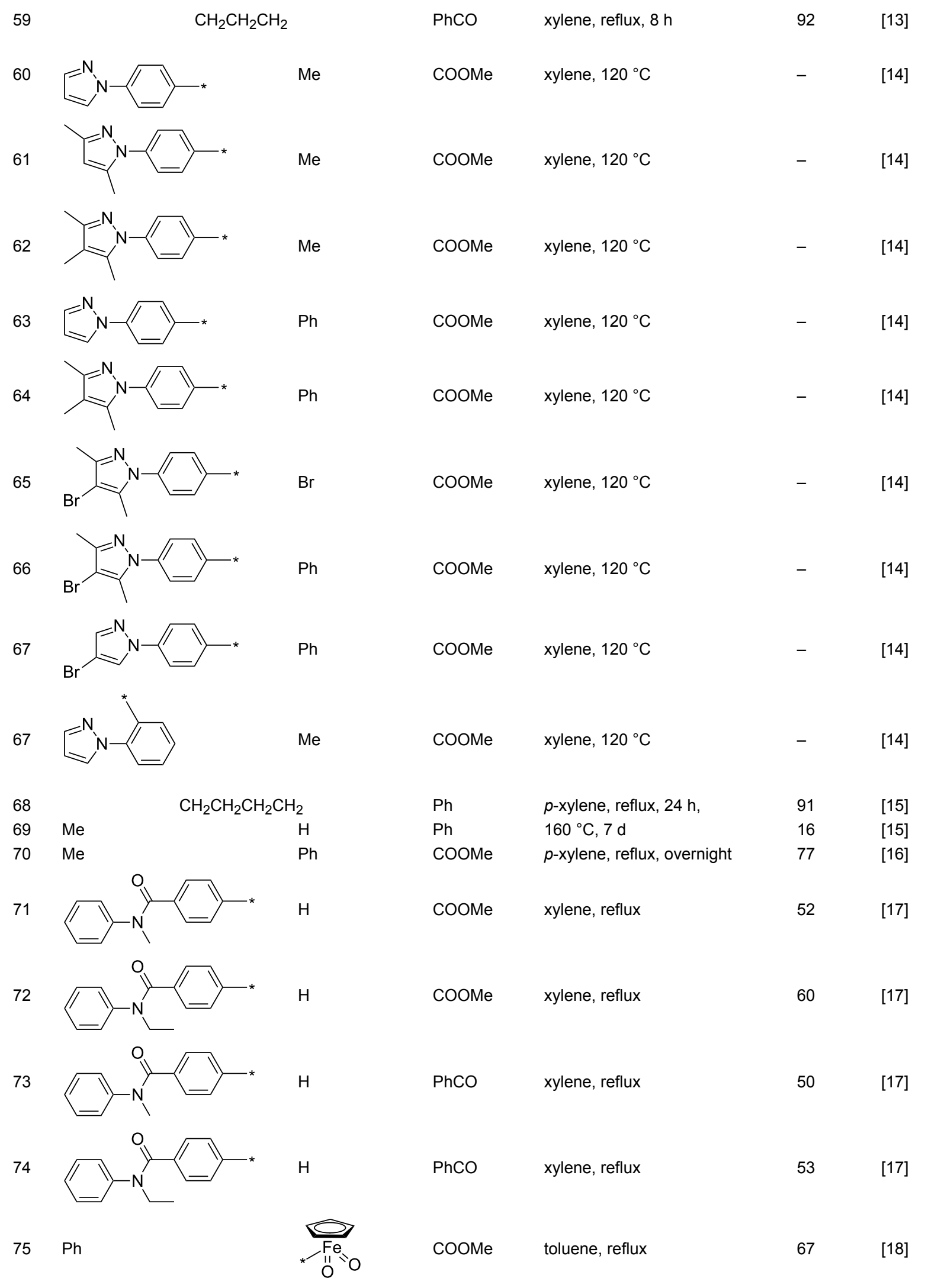


Table 1: Thermal cycloaddition of sydnones with symmetrical non-cyclic alkynes. (continued)

$76 \mathrm{Ph}$

$77 \quad \mathrm{Me}$

$78 \mathrm{Ph}$

$79 \mathrm{Me}$

$80 \mathrm{CH}_{2} \mathrm{CH}_{2} \mathrm{CN}$

$81 \mathrm{Ph}$

82 2-Me-Ph

83 2-Et-Ph

84 2-MeO-Ph

85 3-MeO-Ph

86 4-MeO-Ph

87 2-Me-Ph

88 2-Et-Ph

89 2,4-diMe-Ph

90 2,4-diMe-6-Br-Ph

91 2,4-diBr-6-Cl-Ph

92 2-Br-4,6-diMe-Ph

93 4-Br-2-Me-Ph

94 4-Br-2-Me-Ph

96 4-Br-2-Me-Ph

97 4-Br-2-Me-Ph

98 4,6-- $\mathrm{Br}_{2}-2-\mathrm{Me}-\mathrm{Ph}$

99 4-Br-2-Me-Ph

100 2,4-Br $2-6-\mathrm{Me}-\mathrm{Ph}$

101 2-Cl-Ph

102 2-Cl-4-Br-Ph

103 2-Cl-4-Br-Ph

104 4-Br-2-Et-Ph

105 4-Br-2-Me-Ph

106 2,5-diMe-Ph

107 5-Cl-2-Me-Ph

108 2,5-diMe-Ph

109 5-Cl-2-Me-Ph

110 2,4-diMe-Ph

111 2,4-diMe-Ph

112 2,5-diMe-Ph

113 5-Cl-2-Me-Ph

114 2,4-diMe-Ph

115 4-EtOOC-Ph

116 4-Me-Ph

117 4-EtO-Ph

118 3-Cl-4-Me-Ph

119 3-NO $-4-\mathrm{Me}-\mathrm{Ph}$

120 2,3-diMe-Ph

121 2,3-diMe-Ph

122 2,3-diMe-Ph

123 2,3-diMe-Ph

124 2,3-diMe-Ph

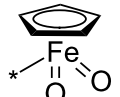

COOt-Bu

toluene, reflux

48

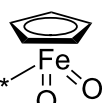

coOMe toluene, reflux

54

$\mathrm{Bu}_{3} \mathrm{Sn} \quad$ xylene, reflux, $16 \mathrm{~h} \quad 98$

COOMe toluene + DMSO, reflux, $6 \mathrm{~h}$

coOMe

coOMe

coOMe

coome

coOMe

coome

coOMe

coome

coOMe

coOMe

coOMe

coOMe

coome

coOMe

coOMe

coOMe

coOMe

coOMe

COOEt

COOMe(Et)

coome

coOMe

coOMe

coome

coOMe

coOMe

coOMe

coOMe

coome

coOMe

coOMe

coOMe

coOMe

coOMe

cOOMe

cOOMe

cOOMe

COOMe

coOMe

coOMe

coOMe

COOMe

COOMe

$\mathrm{COOCH}_{2} \mathrm{CF}_{3}$ toluene, reflux, $12 \mathrm{~h}$

toluene, reflux, $6 \mathrm{~h}$

toluene, reflux, $6 \mathrm{~h}$

toluene, reflux, $6 \mathrm{~h}$

toluene, reflux, $6 \mathrm{~h}$

toluene, reflux, $6 \mathrm{~h}$

toluene, reflux, $6 \mathrm{~h}$

toluene, reflux, $6 \mathrm{~h}$

toluene, reflux, $6 \mathrm{~h}$

toluene, reflux, $6 \mathrm{~h}$

toluene, reflux

toluene, reflux

toluene, reflux

toluene, reflux

xylene, reflux, $8 \mathrm{~h}$

xylene, reflux, $8 \mathrm{~h}$

xylene, reflux, $8 \mathrm{~h}$

xylene, reflux, $8 \mathrm{~h}$

xylene, reflux, $8 \mathrm{~h}$

xylene, reflux, $8 \mathrm{~h}$

xylene, reflux, $3 \mathrm{~d}$

xylene, reflux, $8 \mathrm{~h}$

xylene, reflux, $8 \mathrm{~h}$

xylene, reflux, $8 \mathrm{~h}$

toluene, reflux, $10 \mathrm{~h}$

toluene, reflux, $10 \mathrm{~h}$

toluene, reflux, $8 \mathrm{~h}$

toluene, reflux, $8 \mathrm{~h}$

toluene, reflux, $8 \mathrm{~h}$

toluene, reflux, $8 \mathrm{~h}$

toluene, reflux, $8 \mathrm{~h}$

toluene, reflux, $8 \mathrm{~h}$

toluene, reflux, $8 \mathrm{~h}$

toluene, reflux, $8 \mathrm{~h}$

toluene, reflux, $8 \mathrm{~h}$

$p$-xylene, reflux, overnight

$p$-xylene, reflux, overnight

$p$-xylene, reflux, overnight

xylene, $120^{\circ} \mathrm{C}, 1 \mathrm{~h}$

xylene, $120^{\circ} \mathrm{C}, 1 \mathrm{~h}$

xylene, $120^{\circ} \mathrm{C}, 1 \mathrm{~h}$

toluene, reflux, $10 \mathrm{~h}$

toluene, reflux, $10 \mathrm{~h}$

toluene, reflux, $10 \mathrm{~h}$

toluene, reflux, $10 \mathrm{~h}$

$\mathrm{H}$
[18]

[18]

[19]

[20]

[20]

[20]

[20]

[20]

[20]

[20]

[21]

[21]

[21]

[21]

[22]

[22]

[22]

[22]

[22]

[22]

[22]

[23]

[23]

[24]

[25]

[25]

[25]

[25]

[25]

[25]

[25]

[25]

[25]

[26]

[26]

[26]

[27]

[27]

[27]

[28]

[28]

[28]

[28]

[28] 
Table 1: Thermal cycloaddition of sydnones with symmetrical non-cyclic alkynes. (continued)

\begin{tabular}{|c|c|c|c|c|c|c|}
\hline 125 & $\mathrm{Ph}$ & $\mathrm{Ph}$ & COOMe & toluene, reflux, $16 \mathrm{~h}$ & 99 & [30] \\
\hline 126 & $\mathrm{Ph}$ & $4-\mathrm{NO}_{2}-\mathrm{Ph}$ & COOMe & toluene, reflux, $16 \mathrm{~h}$ & 87 & [30] \\
\hline 127 & $\mathrm{Ph}$ & $4-\mathrm{OCH}_{3}-\mathrm{Ph}$ & COOMe & toluene, reflux, $16 \mathrm{~h}$ & 82 & [30] \\
\hline 128 & $\mathrm{Ph}$ & $\mathrm{CF}_{3}$ & $\mathrm{Ph}$ & o-dichlorobenzene, $24 \mathrm{~h}, 180^{\circ} \mathrm{C}$ & 53 & [32] \\
\hline 129 & $\mathrm{Bn}$ & $\mathrm{CF}_{3}$ & COOMe & $\begin{array}{l}\text { o-dichlorobenzene, } 20 \mathrm{~h}, 120^{\circ} \mathrm{C} \\
\left(180^{\circ} \mathrm{C}\right)\end{array}$ & $\begin{array}{l}54^{\mathrm{a}} \\
(51)^{\mathrm{a}}\end{array}$ & [33] \\
\hline 130 & $\mathrm{Ph}$ & $\mathrm{CH}_{2} \mathrm{~F} \rightarrow \mathrm{CH}_{2} \mathrm{OH}$ & COOMe & o-dichlorobenzene, $24 \mathrm{~h}, 100^{\circ} \mathrm{C}$ & 57 & [33] \\
\hline 131 & $\mathrm{Ph}$ & $\mathrm{OH}$ & COOMe & $\begin{array}{l}\text { o-dichlorobenzene, } 5 \mathrm{~min}, \\
180^{\circ} \mathrm{C} \text { ( } \mu \text {-wave) }\end{array}$ & 92 & [34] \\
\hline 132 & 4-MeO-Ph & $\mathrm{OH}$ & coOMe & $\begin{array}{l}\text { o-dichlorobenzene, } 20 \mathrm{~min}, \\
180^{\circ} \mathrm{C} \text { ( } \mu \text {-wave) }\end{array}$ & 60 & [34] \\
\hline 133 & & & coOMe & xylene, reflux, $3 \mathrm{~h}$ & 70 & [36] \\
\hline 134 & & & coOMe & toluene, $115^{\circ} \mathrm{C}$, overnight & 81 & [52] \\
\hline 135 & 2-MeO-Ph & $\mathrm{H}, \mathrm{Br}, \mathrm{Cl}$ & COOMe & xylene, reflux & n.d. & [37] \\
\hline 136 & $2-\mathrm{NO}_{2}-\mathrm{Ph}$ & $\mathrm{H}, \mathrm{Br}, \mathrm{Cl}$ & coOMe & xylene, reflux & n.d. & [37] \\
\hline 137 & $3-\mathrm{NO}_{2}-\mathrm{Ph}$ & $\mathrm{H}, \mathrm{Br}, \mathrm{Cl}$ & COOMe & xylene, reflux & n.d. & [37] \\
\hline 138 & 2-Cl-Ph & $\mathrm{H}, \mathrm{Br}, \mathrm{Cl}$ & COOMe & xylene, reflux & n.d. & [37] \\
\hline 139 & 3-Cl-Ph & $\mathrm{H}, \mathrm{Br}, \mathrm{Cl}$ & coOMe & xylene, reflux & n.d. & [37] \\
\hline 140 & $\mathrm{Ph}$ & $\mathrm{Ph}$ & $\mathrm{PhCO}$ & toluene, heating, $92 \mathrm{~h}$ & 69 & [38] \\
\hline 141 & $\mathrm{Ph}$ & $\mathrm{H}$ & $\mathrm{PhCO}$ & PEG, $115^{\circ} \mathrm{C}, 3 \mathrm{~min},(\mu$-wave) & 50 & [39] \\
\hline 142 & 4-Cl-Ph & $\mathrm{H}$ & $\mathrm{PhCO}$ & PEG, $115^{\circ} \mathrm{C}, 3 \mathrm{~min}$, ( $\mu$-wave) & 51 & [39] \\
\hline 143 & 4-Me-Ph & $\mathrm{H}$ & $\mathrm{PhCO}$ & PEG, $115^{\circ} \mathrm{C}, 3 \mathrm{~min},(\mu$-wave $)$ & 54 & [39] \\
\hline 144 & $\mathrm{Ph}$ & $\mathrm{H}$ & 4-MeOPhCO & PEG, $115^{\circ} \mathrm{C}, 3 \mathrm{~min}$, ( $\mu$-wave) & 48 & [39] \\
\hline 145 & 4-Cl-Ph & $\mathrm{H}$ & 4-MeOPhCO & PEG, $115^{\circ} \mathrm{C}, 3 \mathrm{~min}$, ( $\mu$-wave) & 48 & [39] \\
\hline 146 & 4-Me-Ph & $\mathrm{H}$ & 4-MeOPhCO & PEG, $115^{\circ} \mathrm{C}, 3 \mathrm{~min}$, ( $\mu$-wave) & 49 & [39] \\
\hline
\end{tabular}

aMixture of dimethyl 1-benzyl-5-trifluoromethyl-1 $\mathrm{H}$-pyrazole-3,4-dicarboxylate and dimethyl 1-benzyl-3-trifluoromethyl-1 $\mathrm{H}$-pyrazole-4,5-dicarboxylate in the ratio $96: 4\left(\right.$ at $120^{\circ} \mathrm{C}$ ) or $63: 37$ (at $180^{\circ} \mathrm{C}$ ).

Extraordinarily good dipolarophiles - e.g., cycloalkynes - containing a very reactive "bent" triple bond such as in bicyclo[6.1.0]non-4-yne-9-methanol [42-44] or in 3,3,6,6tetramethylthiacyclohept-4-yne [44] were recently suggested as highly reactive partners for bio-orthogonal ligation reactions $[45,46]$. It is also possible to generate highly unstable cyclopentyne or cyclohexyne in situ from the corresponding 2-trimethylsilylcycloalken-1-yl triflates [47] and trap them by reaction with sydnones (Scheme 2).

These strain-promoted reactions proceed quickly under very mild conditions (at room temperature, in aqueous phosphate buffer with solubilizing DMSO). In a similar manner, very reactive benzynes (didehydrobenzenes) generated either from 2-aminobenzoic acid [48], from symmetrically substituted 2-trimethylsilylphenyl triflates [49-52] or from 2-(trimethylsilyl)phenyl trimethylsilyl ethers [53] react with sydnones in $\mathrm{MeCN}$ or THF giving 2-substituted $2 \mathrm{H}$-indazoles in good to excellent yields (40-99\%) at room temperature (Scheme 3).

It was also observed, that formation of isomeric pyrazole-4,5dicarboxylates (B) can sometimes accompany the production of pyrazole-3,4-dicarboxylates (A) under thermal conditions [33] although their formation is not photoinduced (cf. next chapter) because the reaction also takes place in the absence of light. Depending on the temperature, a new reaction pathway involving benzylic group migration, $\mathrm{CO}_{2}$ extrusion and final cycloaddition was proposed (Scheme 4). 


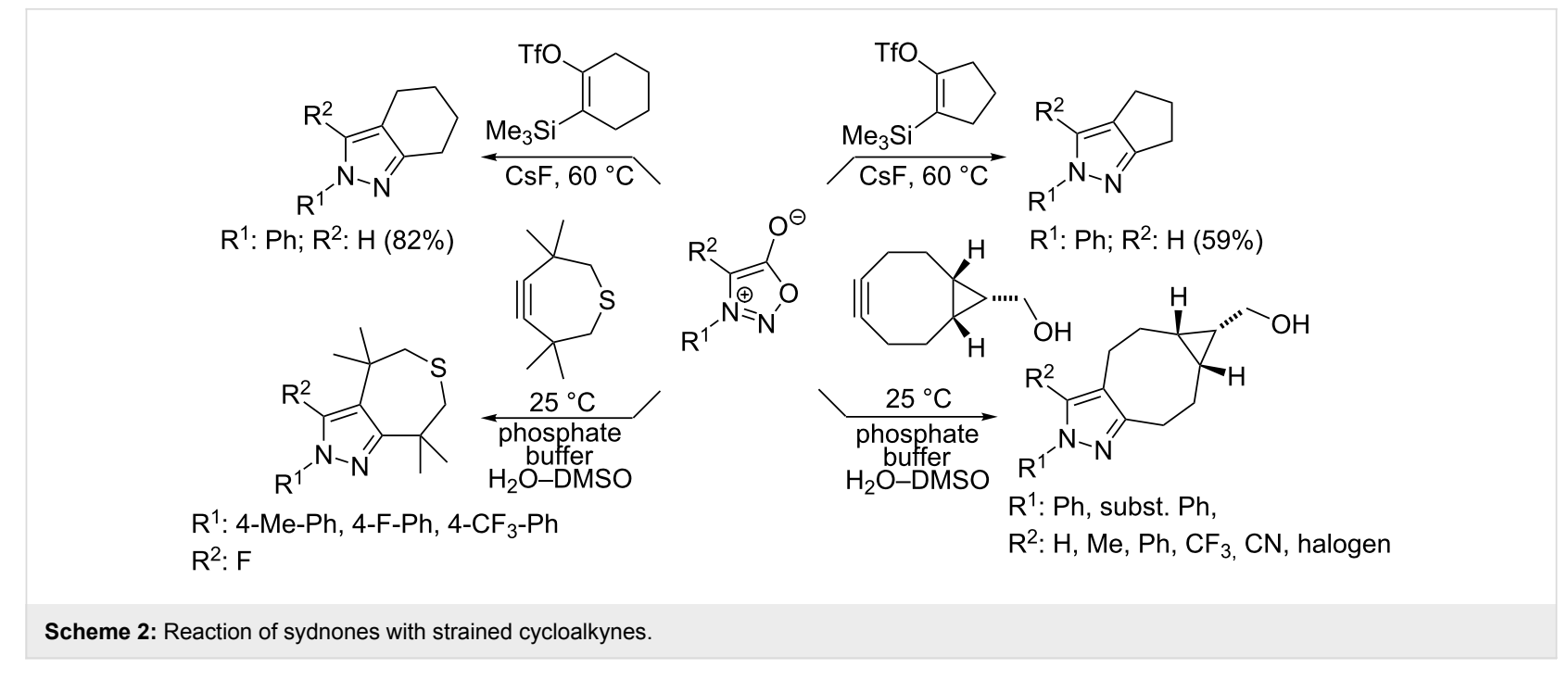

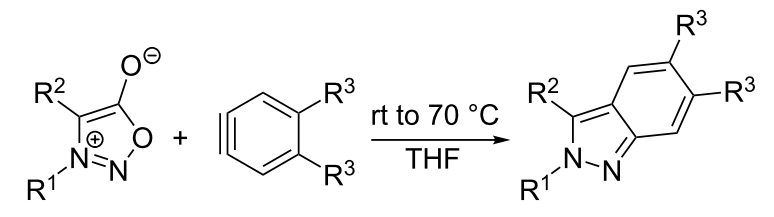

$\mathrm{R}^{1}: \mathrm{Ph}, 4-\mathrm{MeO}-\mathrm{Ph}, 4-\mathrm{Me}-\mathrm{Ph}, 4-\mathrm{Br}-\mathrm{Ph}, 4-\mathrm{Cl}-\mathrm{Ph}, 4-\mathrm{Me}-\mathrm{Ph}, \mathrm{Ph}, 3,4-\mathrm{CH}_{2} \mathrm{O}_{2}-\mathrm{Ph}, \mathrm{Me}, \mathrm{Bn}$

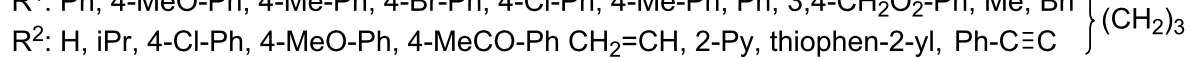

$\mathrm{R}^{3}$ : Me, MeO, cyclopent-3-en-1,4-diyl

Scheme 3: Reaction of sydnones with didehydrobenzenes.

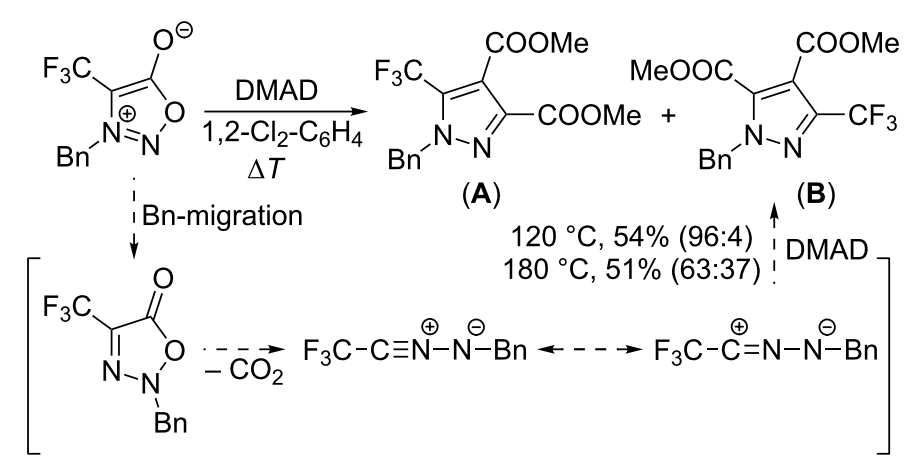

Scheme 4: Formation of isomeric pyrazole dicarboxylates.

\section{Kinetics and mechanism of thermal cycloaddition}

The kinetics and reaction mechanism of the thermal cycloaddition between 4-methyl-3-phenylsydnone and DMAD was first studied by Huisgen and Gothardt [54] in $p$-cymene at $90-110^{\circ} \mathrm{C}$. They found the cycloaddition to be overall second order and its activation entropy $\Delta S^{\neq}=-130 \mathrm{~J} \cdot \mathrm{mol}^{-1} \cdot \mathrm{K}^{-1}$ showed association character of the rate-limiting step with a rel- atively tight transition state. Moreover, for the cycloaddition of the structurally similar ethyl phenylpropiolate in various solvents only a small decrease of the bimolecular rate constant with increasing solvent polarity (in terms of relative permittivity) was observed excluding a transition state having a polarized character. Finally, substitution effects in the 3-(4-substituted phenyl) group of sydnones were studied and a relatively low Hammett reaction constant $\rho \approx+0.8$ was estimated from 
four derivatives $(\mathrm{MeO}, \mathrm{Me}, \mathrm{H}$ and $\mathrm{Cl})$. An even smaller dependence of the rate constants on the solvent polarity and substituent effect sensitivity ( $\rho \approx+0.3$ to +0.4 ) was described [55] for reactions of 3-(4-substituted phenyl)sydnones with more reactive DMAD while the activation entropy $\left(\Delta S^{\not}=-106\right.$ to $\left.-121 \mathrm{~J} \cdot \mathrm{mol}^{-1} \cdot \mathrm{K}^{-1}\right)$ remained similar. The reaction mechanism (Scheme 5) consistent with these kinetic measurements involves rate-limiting formation of a bicyclic intermediate via a concerted [3+2]-cycloaddition followed by its very fast decomposition (extrusion of $\mathrm{CO}_{2}$ ) via a retroDiels-Alder [4 + 2]-cycloaddition. The almost spontaneous extrusion of $\mathrm{CO}_{2}$ is caused by an energetically favorable aromatization occurring in this step leading to the formation of the stable pyrazole ring. Both reaction steps are also compatible with Woodward-Hoffmann rules, taking into account orbital symmetry considerations [56].

Three types of [3+2]-cycloadditions (labelled I-III) are known from the literature [57] each differing in the frontier molecular orbital energies between the dipole and dipolarophile. While for type I (HOMO-controlled) combining a high-lying dipole HOMO with a dipolarophile LUMO the reaction is accelerated by electron-donating substituents on the dipole and electronwithdrawing substituents on the dipolarophile (both lowering the HOMO-LUMO energy gap), for type III (LUMO-controlled) combining a low-lying dipole LUMO and a dipolarophile HOMO where substituent effects are completely opposite. For type II cycloadditions in which two-way interac- tions between the dipole HOMO and the dipolarophile LUMO or the dipole LUMO and the dipolarophile HOMO are possible - due to similar energy gaps - both electron-rich as well as electron-poor dipolarophiles/dipoles react more quickly than parent (unsubstituted) ones. Using semi-empirical quantum calculations (CNDO/2), Houk et al. [58] calculated average HOMO/ LUMO energies for azomethine-imines $\left(\varepsilon_{\mathrm{HOMO}}=-8.6 \mathrm{eV}\right.$ and $\varepsilon_{\text {LUMO }}=0.3 \mathrm{eV}$ ) and predicted that the $\varepsilon_{\text {LUMO }}$ for structurally related sydnones containing an electron-withdrawing -COO- motif should be even much lower suggesting a LUMOcontrolled reaction (type III). Such a prediction seems to be correct for reaction of 4-(substituted phenyl)sydnones with DMAD for which positive Hammett $\rho$-values were observed [54,55]. On the other hand Huisgen and Gotthardt [54] measured bimolecular rate constants for the above-mentioned reaction of 4-methyl-3-phenylsydnone and various acetylenes in $p$-cymene at $140{ }^{\circ} \mathrm{C}$ (Table 2) and found a reactivity sequence corresponding rather to type II or even type I cycloadditions.

The most reactive were electron-poor alkynes (acetylene(di)carboxylates, benzoyl phenylacetylene) while electron-rich alkynes (tetradec-1-yne, 1-phenylpropyne) were much less reactive. Unfortunately, the reaction rate constant was not measured for the reaction with acetylene itself. However, on the basis of the published $[1,2]$ synthetic protocol (acetone, $170{ }^{\circ} \mathrm{C}, 25 \mathrm{~h}$ ) it appears that this cycloaddition is very slow and requires a higher temperature.
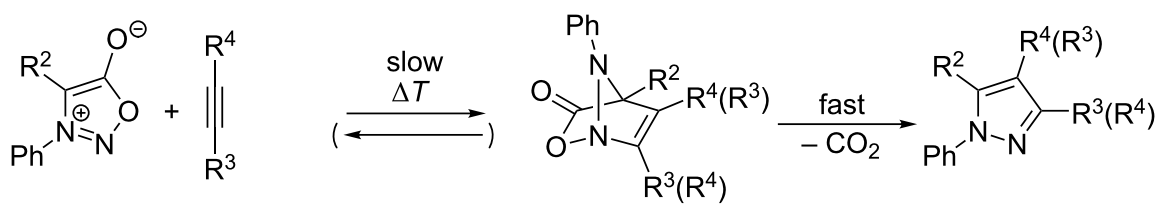

$\mathrm{R}^{2}: \mathrm{Me} ; \mathrm{R}^{3}=\mathrm{R}^{4}:$ COOMe [54]

$R^{2}: M e ; R^{3}$ and $R^{4}: P h$ and COOEt [54]

$\mathrm{R}^{2}: \mathrm{H} ; \mathrm{R}^{3}=\mathrm{R}^{4}$ : COOMe [55]

Scheme 5: Mechanism of thermal cycloaddition between sydnones and alkynes.

\begin{tabular}{|c|c|c|c|}
\hline $\begin{array}{l}\text { dipolarophile } \\
\text { (disubstituted alkyne) }\end{array}$ & $\begin{array}{l}10^{5} \mathrm{k} \\
\left(\mathrm{L} \cdot \mathrm{mol}^{-1} \cdot \mathrm{s}^{-1}\right)\end{array}$ & $\begin{array}{l}\text { dipolarophile } \\
\text { (monosubstituted alkyne) }\end{array}$ & $\begin{array}{l}10^{5} \mathrm{k} \\
\left(\mathrm{L} \cdot \mathrm{mol}^{-1} \cdot \mathrm{s}^{-1}\right)\end{array}$ \\
\hline MeOOC-C $\equiv \mathrm{C}-\mathrm{COOMe}$ & 2580 & $\mathrm{H}-\mathrm{C} \equiv \mathrm{C}-\mathrm{COOMe}$ & 823 \\
\hline $\mathrm{Ph}-\mathrm{C} \equiv \mathrm{C}-\mathrm{COPh}$ & 135 & $\mathrm{H}-\mathrm{C} \equiv \mathrm{C}-\mathrm{CH}(\mathrm{OPr})_{2}$ & 39 \\
\hline $\mathrm{Ph}-\mathrm{C} \equiv \mathrm{C}-\mathrm{COOEt}$ & 99 & $\mathrm{H}-\mathrm{C} \equiv \mathrm{C}-\mathrm{Ph}$ & 18 \\
\hline $\mathrm{Ph}-\mathrm{C} \equiv \mathrm{C}-\mathrm{Ph}$ & $3.0^{\mathrm{a}}$ & $\mathrm{H}-\mathrm{C} \equiv \mathrm{C}-\left(\mathrm{CH}_{2}\right)_{11} \mathrm{CH}_{3}$ & 6.0 \\
\hline $\mathrm{Ph}-\mathrm{C} \equiv \mathrm{C}-\mathrm{Me}$ & 1.9 & & \\
\hline
\end{tabular}

an decaline. 
Recently [42-44], a kinetic investigation was performed for the cycloaddition of various sydnones with strained cycloalkynes such as bicyclo[6.1.0]non-4-yne-9-methanol (BCN) or 3,3,6,6tetramethylthiacyclohept-4-yne (TMTH). It was found that the reaction of $\mathrm{BCN}$ with 3-(4-substituted phenyl)sydnones roughly obeys a Hammett correlation with $\rho \approx+1.35 \pm 0.25$ [43] thus indicating a type III mechanism. However, the effect of substituent in position 4- of 3-phenylsydnone is ambiguous. While all halogens substantially accelerate the reaction rate $(\mathrm{F}>\mathrm{Cl}>\mathrm{Br}$ $>$ I) other substituents cause up to tenfold deceleration $(\mathrm{H}>\mathrm{Me}$ $>\mathrm{CF}_{3}>\mathrm{CN}$ ) regardless of their polar effects $[43,44]$. Steric factors cannot explain the influence of 4 -substituent because 4-phenylsydnone reacts equally as unsubstituted one. The most reactive 4-fluoro-3-phenylsydnones [44] were found to react with BCN and TMTH in two kinetically independent reaction steps corresponding to fast formation of the addition intermediate and its slow decomposition to pyrazole and $\mathrm{CO}_{2}$. Such ambiguous substitution effects are therefore worthy of further investigations.

\section{Photochemical reaction of sydnones with symmetrical alkynes}

In 1966 Krauch et al. [59] dealt with irradiation (using a highpressure $\mathrm{Hg}$ lamp) of benzene or dioxane solutions of 3-phenylsydnone and proposed formation of $\mathrm{N}$-phenylnitrilimine as the main reaction product via an internal ring closure, extrusion of $\mathrm{CO}_{2}$ and ring opening (Scheme 6). This very reactive 1,3-dipole was trapped by reaction with external ( ${ }^{14} \mathrm{C}$-labelled) $\mathrm{CO}_{2}$ to give 3-phenyl-1,3,4-oxadiazol-2(3H)-one (Scheme 6). A similar experiment was performed by Ohta et al. [60] five years later who irradiated single 3,4-diphenylsydnones and obtained the corresponding 2,4,5-triphenyl-1,2,3-triazoles in 21-24\% yields (first misinterpreted as 1,3-diphenyldiazirine [61]). In the same year Angadiyavar and George [62], Gotthardt and Reiter $[63,64]$ and Märky, Hansen and Schmid [65] found that irradiation of a mixture of 3-phenylsydnone or 3,4-diphenylsydnones together with DMAD gave different isomeric [3+2]-cycloadducts (pyrazole-4,5-dicarboxylates) than what were obtained under thermal conditions and proved the reaction pathway to proceed via the corresponding $\mathrm{N}$-phenylnitrilimine.

The yields (Table 3) are generally lower than those of reactions performed under thermal conditions - most probably due to the lower stability of the key intermediate $-\mathrm{N}$-phenylnitrilimine which can undergo dimerization or reverse trapping of evolved $\mathrm{CO}_{2}$. Yields are always much better for 3,4-diarylsydnones for which the corresponding $N$-phenylnitrilimine is resonancestabilized. The yields also depend on the photoreactor construction [64]. For example 1,3-diphenylsydnone reacts with DMAD in a batch reactor (Rayonet) under $300 \mathrm{~nm}$ irradiation to give only $29 \%$ of dimethyl 1,3-diphenylpyrazole-4,5-dicarboxylate while in a wetted-wall photo reactor (Normag) the yield is increased up to $84 \%$ (at $17{ }^{\circ} \mathrm{C}$ in DCM).

\section{Thermal reaction of sydnones with terminal alkynes}

As early as in his first work [1] dealing with sydnone-alkyne cycloaddition Huisgen et al. found that some non-symmetrical alkynes (oct-1-yne, phenylacetylene and especially methyl propiolate) gave mixture of both pyrazole regioisomers. The following Table 4 summarizes all known examples $[1,2,8,20,24,32-34,36,66-93]$ where the ratio of both possible

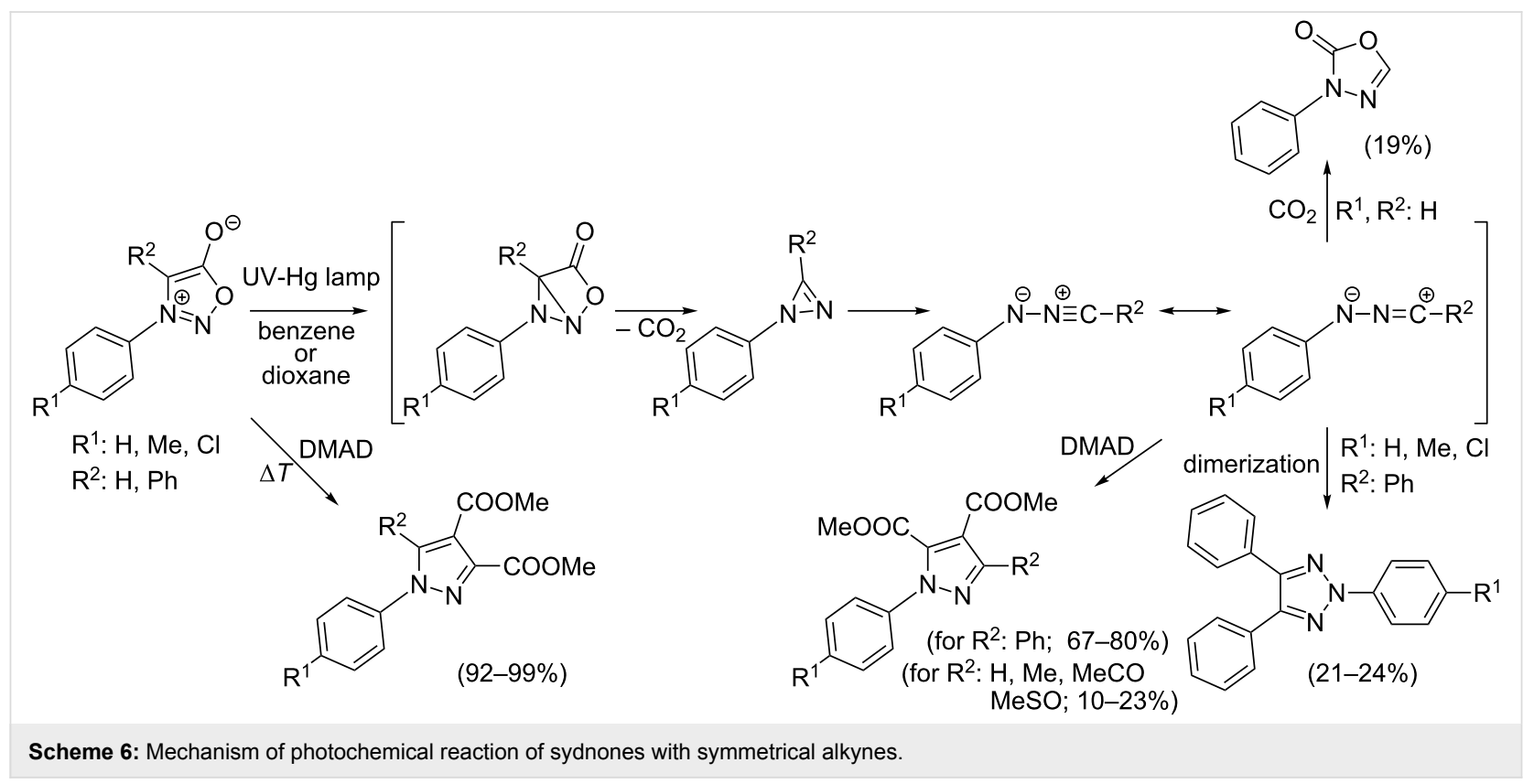




\begin{tabular}{|c|c|c|c|c|c|}
\hline entry & $\mathrm{R}^{1}$ & $R^{2}$ & conditions & yield [\%] & ref. \\
\hline 1 & $\mathrm{Ph}$ & $\mathrm{H}$ & $\mathrm{CH}_{2} \mathrm{Cl}_{2}, 50 \mathrm{~h}$, light $(300 \mathrm{~nm})$ & 10 & {$[63,64]$} \\
\hline 2 & $\mathrm{Ph}$ & $\mathrm{Me}$ & $\mathrm{CH}_{2} \mathrm{Cl}_{2}, 19 \mathrm{~h}$, light (300 nm) & 23 & {$[63,64]$} \\
\hline 3 & $\mathrm{Ph}$ & $\mathrm{Ph}$ & $\begin{array}{l}\text { benzene, } 2 \mathrm{~h} \text {, } \\
\mathrm{CH}_{2} \mathrm{Cl}_{2} \text {, light }(300 \mathrm{~nm}) \text {, batch } \\
\mathrm{CH}_{2} \mathrm{Cl}_{2}, 29.5 \mathrm{~h} \text {, light }(300 \mathrm{~nm}) \\
\text { wetted-wall photoreactor } \\
\text { dioxane, } \mathrm{Hg} \text { lamp }\end{array}$ & $\begin{array}{l}67 \\
29 \\
29 \\
84 \\
\text { ca. } 80\end{array}$ & $\begin{array}{l}{[62]} \\
{[63][64]} \\
{[64]} \\
{[65]}\end{array}$ \\
\hline 4 & $\mathrm{Ph}$ & 4-Me-Ph & $\mathrm{CH}_{2} \mathrm{Cl}_{2}, \mathrm{Hg}$ lamp & ca. 80 & [65] \\
\hline 5 & $\mathrm{Ph}$ & MeS & benzene, $25 \mathrm{~h}, \mathrm{Hg}$ lamp & 45 & [64] \\
\hline 6 & $\mathrm{Ph}$ & MeSO & $\mathrm{CH}_{2} \mathrm{Cl}_{2}, 110 \mathrm{~h}, \mathrm{Hg}$ lamp & 12 & [64] \\
\hline 7 & $\mathrm{Ph}$ & $\mathrm{MeCO}$ & benzene, $41 \mathrm{~h}, \mathrm{Hg}$ lamp & 17 & [64] \\
\hline
\end{tabular}

Table 4: Thermal cycloaddition of sydnones with terminal alkynes.

\begin{tabular}{|c|c|c|c|c|c|c|c|}
\hline & & $\overbrace{\mathrm{R}^{1}}^{\mathrm{N}^{\oplus}=\mathrm{N}^{\prime}} \mathrm{O}_{\mathrm{O}}^{\Theta}$ & $\left.\right|_{\mathrm{R}^{3}} ^{\mathrm{H}}$ & $+\mathrm{R}^{1^{-N}}$ & $\hat{\gamma}^{\mathrm{R}^{3}}$ & & \\
\hline entry & $\mathrm{R}^{1}$ & $\mathrm{R}^{2}$ & $\mathrm{R}^{3}$ & conditions & $\begin{array}{l}\text { ratio } \\
1,3: 1,4\end{array}$ & $\begin{array}{l}\text { yield } \\
{[\%]^{a}}\end{array}$ & ref. \\
\hline 1 & $\mathrm{Ph}$ & $\mathrm{Me}$ & $n$-Hex & xylene, $140^{\circ} \mathrm{C}, 30 \mathrm{~h}$ & n.d. & 78 & [1] \\
\hline 2 & $\mathrm{Ph}$ & $\mathrm{H}$ & $n$-Hex & $\begin{array}{l}\text { toluene, } 111^{\circ} \mathrm{C}, 52 \mathrm{~h} \\
\text { xylene, } 160^{\circ} \mathrm{C}, 24 \mathrm{~h}\end{array}$ & $\begin{array}{l}\text { n.d. } \\
90: 10\end{array}$ & $\begin{array}{l}72 \\
65\end{array}$ & $\begin{array}{l}{[2]} \\
{[91]}\end{array}$ \\
\hline 3 & $\mathrm{Ph}$ & $\mathrm{H}$ & $\mathrm{Ph}$ & $\begin{array}{l}\text { chlorobenzene, } 120{ }^{\circ} \mathrm{C}, 20 \mathrm{~h} \\
\text { xylene, } 140^{\circ} \mathrm{C}, 16 \mathrm{~h} \\
o \text {-DCB, } \mu \text {-wave, } 200^{\circ} \mathrm{C}, 2 \mathrm{~h} \\
o \text {-DCB, } 140^{\circ} \mathrm{C}, 24 \mathrm{~h}\end{array}$ & $\begin{array}{l}\text { n.d. } \\
>95: 5 \\
91: 9 \\
91: 9\end{array}$ & $\begin{array}{l}79 /<2 \\
35 \\
66 \\
62\end{array}$ & $\begin{array}{l}{[1,2]} \\
{[82]} \\
{[84]} \\
{[92]}\end{array}$ \\
\hline 4 & $\mathrm{Ph}$ & $\mathrm{Me}$ & $\mathrm{Ph}$ & $\begin{array}{l}140^{\circ} \mathrm{C}, 12 \mathrm{~h} \\
142^{\circ} \mathrm{C}, 7 \mathrm{~h}\end{array}$ & $\begin{array}{l}\sim 80: 20 \\
\sim 89: 11\end{array}$ & $\begin{array}{l}64 / 15 \\
73 / 9\end{array}$ & $\begin{array}{l}{[1]} \\
{[2]}\end{array}$ \\
\hline 5 & $\mathrm{Bn}$ & $\mathrm{H}$ & $\mathrm{Ph}$ & xylene, $135-140^{\circ} \mathrm{C}, 20 \mathrm{~h}$ & $100: 0$ & $69-74$ & {$[1,2]$} \\
\hline 6 & $\mathrm{Ph}$ & $\mathrm{H}$ & coOMe & $\begin{array}{l}\text { xylene, } 100^{\circ} \mathrm{C}, 48 \mathrm{~h} \\
\text { sc- } \mathrm{CO}_{2}, 60-160^{\circ} \mathrm{C}, 7.6 \mathrm{MPa}\end{array}$ & $\begin{array}{l}76: 24 \\
85: 15-76: 24\end{array}$ & $\begin{array}{l}70 / 22 \\
-\end{array}$ & $\begin{array}{l}{[1,2]} \\
{[93]}\end{array}$ \\
\hline 7 & $\mathrm{Ph}$ & $\mathrm{Me}$ & COOMe & $\begin{array}{l}140^{\circ} \mathrm{C}, 4 \mathrm{~h} \\
\text { xylene, reflux, } 1 \mathrm{~h}\end{array}$ & $\begin{array}{l}\text { n.d. } \\
65: 35\end{array}$ & $\begin{array}{l}61 / 10 \\
55 / 29\end{array}$ & $\begin{array}{l}{[1]} \\
{[2]}\end{array}$ \\
\hline 8 & $\mathrm{Ph}$ & $\mathrm{H}$ & $\mathrm{CH}(\mathrm{OPr})_{2}$ & xylene, $135-140{ }^{\circ} \mathrm{C}, 3 \mathrm{~h}$ & n.d. & $28 / 58$ & [2] \\
\hline 9 & $\mathrm{Ph}$ & $\mathrm{Me}$ & $\mathrm{CH}(\mathrm{OPr})_{2}$ & xylene, $135-140^{\circ} \mathrm{C}, 15 \mathrm{~h}$ & n.d. & 77 & {$[1]$} \\
\hline 10 & $\mathrm{Bn}$ & $\mathrm{H}$ & $\mathrm{CH}(\mathrm{OPr})_{2}$ & xylene, $135-140^{\circ} \mathrm{C}, 15 \mathrm{~h}$ & n.d & 78 & [2] \\
\hline 11 & $\mathrm{Ph}$ & $\mathrm{H}$ & $\mathrm{CH}_{2} \mathrm{OH}$ & reflux, $24 \mathrm{~h}$ & $100: 0$ & $66-72$ & {$[1,2]$} \\
\hline 12 & $\mathrm{Ph}$ & $\mathrm{H}$ & $\mathrm{CN}$ & chlorobenzene, $110^{\circ} \mathrm{C}, 24 \mathrm{~h}$ & $100: 0$ & 50 & {$[66]$} \\
\hline 13 & $\mathrm{NMe}_{2}$ & $\mathrm{H}$ & $\mathrm{Ph}$ & tetraline, reflux, $5 \mathrm{~h}$ & n.d. & $60 /-$ & {$[67]$} \\
\hline 14 & $\mathrm{NMe}_{2}$ & $\mathrm{H}$ & 4-Cl-Ph & tetraline, reflux, $5 \mathrm{~h}$ & n.d. & $23 /-$ & [67] \\
\hline 15 & $\mathrm{NMe}_{2}$ & $\mathrm{H}$ & 4-Me-Ph & tetraline, reflux, $5 \mathrm{~h}$ & n.d. & $32 /-$ & [67] \\
\hline 16 & $\mathrm{NMe}_{2}$ & $\mathrm{H}$ & $n$-Hex & tetraline, reflux, $5 \mathrm{~h}$ & n.d. & $50 /-$ & [67] \\
\hline 17 & $\mathrm{O}\left(\mathrm{CH}_{2} \mathrm{CH}_{2}\right)_{2} \mathrm{~N}$ & $\mathrm{H}$ & $\mathrm{Ph}$ & tetraline, reflux, $5 \mathrm{~h}$ & n.d. & $22 /-$ & [67] \\
\hline 18 & $\left(\mathrm{CH}_{2}\right)_{5} \mathrm{~N}$ & $\mathrm{H}$ & $4-\mathrm{Cl}-\mathrm{Ph}$ & tetraline, reflux, $5 \mathrm{~h}$ & n.d. & $24 / 1$ & {$[67]$} \\
\hline 19 & $\mathrm{NMe}_{2}$ & $\mathrm{Me}$ & $\mathrm{Ph}$ & tetraline, reflux, $5 \mathrm{~h}$ & n.d. & $81 / 10$ & [67] \\
\hline 20 & $\mathrm{NMe}_{2}$ & $\mathrm{Me}$ & $4-\mathrm{Cl}-\mathrm{Ph}$ & tetraline, reflux, $5 \mathrm{~h}$ & n.d. & $30 / 4$ & [67] \\
\hline 21 & $\mathrm{NMe}_{2}$ & $\mathrm{O}\left(\mathrm{CH}_{2} \mathrm{CH}_{2}\right)_{2} \mathrm{NCH}_{2}$ & $\mathrm{Ph}$ & tetraline, reflux, $5 \mathrm{~h}$ & n.d. & $34 / 2$ & {$[67]$} \\
\hline 22 & $\mathrm{NMe}_{2}$ & $\mathrm{O}\left(\mathrm{CH}_{2} \mathrm{CH}_{2}\right)_{2} \mathrm{NCH}_{2}$ & $4-\mathrm{Cl}-\mathrm{Ph}$ & tetraline, reflux, $5 \mathrm{~h}$ & n.d. & $12 / 2$ & [67] \\
\hline 23 & $\mathrm{Ph}$ & MeS & coOMe & toluene, $95-105^{\circ} \mathrm{C}, 12.5 \mathrm{~h}$ & $46: 54$ & $39 / 50$ & [8] \\
\hline 24 & $\mathrm{Ph}$ & PhS & COOMe & xylene, $140{ }^{\circ} \mathrm{C}, 35 \mathrm{~h}$ & $53: 47$ & 95 & [8] \\
\hline
\end{tabular}




\begin{tabular}{|c|c|c|c|c|c|c|c|}
\hline 25 & $\mathrm{Ph}$ & MeSO & COOMe & mesitylene, $135-140^{\circ} \mathrm{C}, 19 \mathrm{~h}$ & $81: 19$ & $65 / 15$ & [8] \\
\hline 26 & $\mathrm{Ph}$ & $\mathrm{MeCO}$ & COOMe & mesitylene, $155-160{ }^{\circ} \mathrm{C}, 90 \mathrm{~h}$ & $60: 40$ & $46 / 37$ & [8] \\
\hline 27 & $\mathrm{Ph}$ & $\mathrm{Ph}$ & COOMe & $\begin{array}{l}\text { xylene, } 110-115^{\circ} \mathrm{C}, 12 \mathrm{~h} \\
o-D C B, \text { reflux, } 48 \mathrm{~h}\end{array}$ & $\begin{array}{l}50: 50 \\
50: 50\end{array}$ & $\begin{array}{l}40 / 44 \\
97\end{array}$ & $\begin{array}{l}{[8]} \\
{[80]}\end{array}$ \\
\hline 28 & $4-\mathrm{NO}_{2}-\mathrm{Ph}$ & $\mathrm{Ph}$ & COOMe & toluene, $95-105^{\circ} \mathrm{C}, 16 \mathrm{~h}$ & $56: 44$ & $51 / 37$ & [8] \\
\hline 29 & 2,4-diNO $2-\mathrm{Ph}$ & $\mathrm{Ph}$ & COOMe & toluene, $100-105^{\circ} \mathrm{C}, 18.5 \mathrm{~h}$ & $61: 39$ & $55 / 36$ & [8] \\
\hline 30 & $4-\mathrm{NO}_{2}-\mathrm{Ph}$ & $\mathrm{H}$ & COOMe & toluene, $95-105^{\circ} \mathrm{C}, 4 \mathrm{~h}$ & $86: 14$ & 99 & {$[8]$} \\
\hline 31 & $\mathrm{Ph}$ & $\mathrm{H}$ & $\mathrm{PhSO}_{2}$ & toluene, $100^{\circ} \mathrm{C}, 24 \mathrm{~h}$ & $25: 75$ & 56 & {$[68]$} \\
\hline 32 & & $\mathrm{H}_{2} \mathrm{CH}_{2}$ & $\mathrm{Ph}$ & xylene & $\approx 75: 25$ & $51 / 18$ & [69] \\
\hline 33 & $\mathrm{Ph}$ & I & COOMe & xylene, reflux, $24 \mathrm{~h}$ & $58: 42$ & n.d. & {$[20]$} \\
\hline 35 & 2-Et-Ph & $\mathrm{I}$ & COOMe & xylene, reflux, 24 h & $56: 44$ & n.d. & {$[20]$} \\
\hline 36 & $\mathrm{Me}$ & $\mathrm{H}$ & coOMe & toluene, reflux, $12 \mathrm{~h}$ & $100: 0$ & 75 & [70] \\
\hline 37 & $\mathrm{CH}_{2}$ & ${ }_{2} \mathrm{CH}_{2} \mathrm{CH}_{2}$ & COOMe & $\begin{array}{l}\text { xylene, reflux, } 10 \mathrm{~h} \\
\text { xylene, reflux, } 16 \mathrm{~h} \\
\text { xylene, reflux, } 6 \mathrm{~h}\end{array}$ & $\begin{array}{l}67: 33 \\
\text { n.d. } \\
\text { n.d. }\end{array}$ & $\begin{array}{l}60 \\
65 / 26 \\
56 /-\end{array}$ & $\begin{array}{l}{[71]} \\
{[77]} \\
{[83]}\end{array}$ \\
\hline 38 & $\mathrm{CH}_{2}$ & ${ }_{2} \mathrm{CH}_{2} \mathrm{CH}_{2}$ & COOEt & xylene, reflux, $10 \mathrm{~h}$ & $75: 25$ & 75 & {$[71]$} \\
\hline 39 & $\mathrm{CH}_{2}$ & ${ }_{2} \mathrm{CH}_{2} \mathrm{CH}_{2}$ & $\mathrm{COOn-Bu}$ & xylene, reflux, $10 \mathrm{~h}$ & $63: 37$ & 72 & {$[71]$} \\
\hline 40 & $\mathrm{CH}_{2}$ & $\mathrm{CH}_{2} \mathrm{CH}_{2}$ & COOBn & xylene, reflux, $10 \mathrm{~h}$ & $69: 31$ & 59 & [71] \\
\hline 41 & $\mathrm{CH}_{2}$ & $\mathrm{CH}_{2} \mathrm{CH}_{2}$ & $\mathrm{COO}(1-\mathrm{PhEt})$ & xylene, reflux, $10 \mathrm{~h}$ & $66: 34$ & 60 & {$[71]$} \\
\hline 42 & 4-Br-2-Et-Ph & I & COOEt & xylene, reflux, $24 \mathrm{~h}$ & - & - & [24] \\
\hline 43 & & & COOMe & o-xylene, reflux, $15 \mathrm{~h}$ & n.d. & $68 / 12$ & {$[72,77]$} \\
\hline 44 & & & COOMe & $\begin{array}{l}\text { o-xylene, reflux, } 19 \mathrm{~h} \\
\text { xylene, reflux, } 4 \mathrm{~h}\end{array}$ & n.d. & $\begin{array}{l}49 / 22 \\
53 / 21\end{array}$ & $\begin{array}{l}{[72,73]} \\
{[36]}\end{array}$ \\
\hline 45 & & & COOMe & o-xylene, reflux, $16 \mathrm{~h}$ & n.d. & $64 / 24$ & {$[72,77]$} \\
\hline 46 & & & COOMe & o-xylene, reflux, $16 \mathrm{~h}$ & n.d. & $32 / 32$ & {$[72]$} \\
\hline 47 & & & COOMe & $\begin{array}{l}\text { o-xylene, reflux, } 16 \mathrm{~h} \\
\text { o-xylene, reflux, } 15 \mathrm{~h}\end{array}$ & n.d. & $\begin{array}{l}59 / 34 \\
68 / 12\end{array}$ & $\begin{array}{l}{[72]} \\
{[73]}\end{array}$ \\
\hline 48 & & & COOMe & o-xylene, reflux, $21 \mathrm{~h}$ & $40: 60$ & 80 & {$[72]$} \\
\hline 49 & & $\mathrm{H}_{2} \mathrm{CH}_{2}$ & COOMe & $\begin{array}{l}\text { xylene, reflux, } 8 \mathrm{~h} \\
\text { 1,2-diethoxyethane, } \\
120-125^{\circ} \mathrm{C}, 8 \mathrm{~h}\end{array}$ & $\begin{array}{l}\text { n.d. } \\
\approx 87: 13\end{array}$ & $\begin{array}{l}40 / 35 \\
47\end{array}$ & $\begin{array}{l}{[13]} \\
{[74]}\end{array}$ \\
\hline 50 & 4-EtO-Ph & $\mathrm{H}$ & COOEt & chlorobenzene, reflux, $48 \mathrm{~h}$ & $76: 24$ & 90 & [75] \\
\hline 51 & 4-EtO-Ph & 1 & COOEt & chlorobenzene, reflux, 48 h & $56: 44$ & 81 & {$[75]$} \\
\hline 52 & 4-EtO-Ph & $\mathrm{CN}$ & COOEt & chlorobenzene, reflux, $48 \mathrm{~h}$ & $58: 42$ & 80 & {$[75,76]$} \\
\hline 53 & 4-EtO-Ph & $\mathrm{CH}_{2} \mathrm{OH}$ & COOEt & chlorobenzene, reflux, $48 \mathrm{~h}$ & $63: 37$ & 71 & {$[75]$} \\
\hline 54 & 4-EtO-Ph & PhS & COOEt & chlorobenzene, reflux, 48 h & $52: 48$ & 71 & {$[75]$} \\
\hline 55 & 4-EtO-Ph & $\mathrm{CN}$ & COOBn & chlorobenzene, reflux, 48 h & $57: 43$ & 76 & {$[75,76]$} \\
\hline 56 & 4-EtO-Ph & $\mathrm{CN}$ & $\mathrm{COO} t-\mathrm{Bu}$ & chlorobenzene, reflux, $48 \mathrm{~h}$ & $78: 22$ & 79 & {$[75,76]$} \\
\hline 57 & 4-EtO-Ph & $\mathrm{CN}$ & $\mathrm{COOCHPh}_{2}$ & chlorobenzene, reflux, $48 \mathrm{~h}$ & $100: 0$ & 85 & {$[75,76]$} \\
\hline $56 a$ & $\mathrm{Ph}$ & $\mathrm{CN}$ & $\mathrm{COOCHPh}_{2}$ & chlorobenzene, reflux, 48 h & $100: 0$ & 80 & {$[75,76]$} \\
\hline $57 a$ & & $\star 2$ & COOMe & o-xylene, reflux, $21 \mathrm{~h}$ & n.d. & 87 & [77] \\
\hline 58 & 2,3-diMe-Ph & $\mathrm{H}$ & COOMe & xylene, reflux, $12 \mathrm{~h}$ & $75: 25$ & n.d. & [28] \\
\hline 59 & $\mathrm{Ph}$ & $\mathrm{H}$ & $\mathrm{Me}_{3} \mathrm{Si}$ & toluene, reflux & n.d. & 95/- & [78] \\
\hline
\end{tabular}









\begin{tabular}{|c|c|c|c|c|c|c|c|}
\hline 104 & $4-\mathrm{NO}_{2}-\mathrm{Ph}$ & $\mathrm{CF}_{3}$ & $\left(\mathrm{CH}_{2}\right)_{3} \mathrm{Cl}$ & $o-D C B, 180^{\circ} \mathrm{C}, 24 \mathrm{~h}$ & $>98: 2$ & 68 & [33] \\
\hline 105 & $\mathrm{Me}$ & $\mathrm{CF}_{3}$ & $\mathrm{Ph}$ & $o-D C B, 180^{\circ} \mathrm{C}, 24 \mathrm{~h}$ & $98: 2$ & 95 & [33] \\
\hline 106 & $\mathrm{Me}$ & $\mathrm{CF}_{3}$ & & $o-D C B, 180^{\circ} \mathrm{C}, 24 \mathrm{~h}$ & $98: 2$ & 82 & [33] \\
\hline 107 & $\mathrm{Me}$ & $\mathrm{CF}_{3}$ & $\mathrm{BnOCH}_{2}$ & $o-\mathrm{DCB}, 180^{\circ} \mathrm{C}, 24 \mathrm{~h}$ & $>98: 2$ & 89 & [33] \\
\hline 108 & $\mathrm{Me}$ & $\mathrm{CF}_{3}$ & COOEt & o-DCB, $180^{\circ} \mathrm{C}, 24 \mathrm{~h}$ & $93: 7$ & 94 & [33] \\
\hline 109 & $\mathrm{Ph}$ & $\mathrm{CF}_{3}$ & BPin & o-DCB, $140^{\circ} \mathrm{C}, 72 \mathrm{~h}$ & $93: 7$ & 69 & {$[32,33]$} \\
\hline 110 & $\mathrm{Me}$ & $\mathrm{CF}_{3}$ & BPin & $\mathrm{o}-\mathrm{DCB}, 140^{\circ} \mathrm{C}, 72 \mathrm{~h}$ & $96: 4$ & 44 & [33] \\
\hline 111 & $\mathrm{Bn}$ & $\mathrm{CF}_{3}$ & $\begin{array}{l}\mathrm{Ph} \text { (2 equiv) } \\
\mathrm{Ph}(2 \text { equiv) } \\
\mathrm{Ph}(2 \text { equiv) } \\
\mathrm{Ph} \text { (10 equiv) }\end{array}$ & $\begin{array}{l}\text { o-DCB, } 180^{\circ} \mathrm{C}, 24 \mathrm{~h} \\
\text { o-DCB, } 140^{\circ} \mathrm{C}, 24 \mathrm{~h} \\
\text { o-DCB, } 140^{\circ} \mathrm{C}, 48 \mathrm{~h} \\
\text { o-DCB, } 180^{\circ} \mathrm{C}, 24 \mathrm{~h}\end{array}$ & $\begin{array}{l}64: 36 \\
96: 4 \\
88: 12 \\
88: 12\end{array}$ & $\begin{array}{l}61 \\
34 \\
66 \\
64\end{array}$ & [33] \\
\hline 112 & $\mathrm{Bn}$ & $\mathrm{CF}_{3}$ & $\mathrm{Bu}$ & o-DCB, $180^{\circ} \mathrm{C}, 24 \mathrm{~h}$ & $72: 28$ & 48 & [33] \\
\hline 113 & $\mathrm{Ph}$ & $\mathrm{CH}_{2} \mathrm{OH}$ & $\mathrm{Ph}$ & $o-D C B, 180^{\circ} \mathrm{C}, 24 \mathrm{~h}$ & n.d. & $72 /-$ & [33] \\
\hline 114 & $\mathrm{Ph}$ & O- & COOEt & $\begin{array}{l}\text { o-DCB, } 180^{\circ} \mathrm{C}, 30 \mathrm{~min}, \\
\mu \text {-wave }\end{array}$ & $88: 12$ & 66 & [34] \\
\hline 115 & $\mathrm{Ph}$ & 0 & $\mathrm{Ph}$ & xylene, $140^{\circ} \mathrm{C}, 6 \mathrm{~h}, \mu$-wave & $98: 2$ & 51 & [34] \\
\hline 116 & $\mathrm{Ph}$ & 0 & $\mathrm{Me}_{3} \mathrm{Si}$ & xylene, $140^{\circ} \mathrm{C}, 3.5 \mathrm{~h}, \mu$-wave & $98: 2$ & 17 & [34] \\
\hline 117 & 4-MeO-Ph & & COOEt & $o$-DCB, $180^{\circ} \mathrm{C}, 1 \mathrm{~h}, \mu$-wave & $83: 17$ & 44 & [34] \\
\hline 118 & $\mathrm{Bn}$ & $\mathrm{O}-\mathrm{OH}$ & COOEt & $\begin{array}{l}\text { o-DCB, } 180^{\circ} \mathrm{C}, 30 \mathrm{~min} \text {, } \\
\mu \text {-wave }\end{array}$ & $67: 33$ & 21 & [34] \\
\hline 119 & $\mathrm{Ph}$ & $\mathrm{H}$ & & toluene, reflux, $12 \mathrm{~h}$ & $100: 0$ & 33 & [86] \\
\hline 120 & 4-Me-Ph & $\mathrm{H}$ & & toluene, reflux, $12 \mathrm{~h}$ & $100: 0$ & 35 & [86] \\
\hline 121 & $4-I-P h$ & $\mathrm{H}$ & & toluene, reflux, $12 \mathrm{~h}$ & $100: 0$ & 40 & [86] \\
\hline 122 & 4-Cl-Ph & $\mathrm{H}$ & & toluene, reflux, $12 \mathrm{~h}$ & $100: 0$ & 43 & [86] \\
\hline 123 & 4-F-Ph & $\mathrm{H}$ & 0 & toluene, reflux, $12 \mathrm{~h}$ & $100: 0$ & 38 & [86] \\
\hline 124 & 4-MeO-Ph & $\mathrm{H}$ & & toluene, reflux, $12 \mathrm{~h}$ & $100: 0$ & 33 & {$[86]$} \\
\hline
\end{tabular}




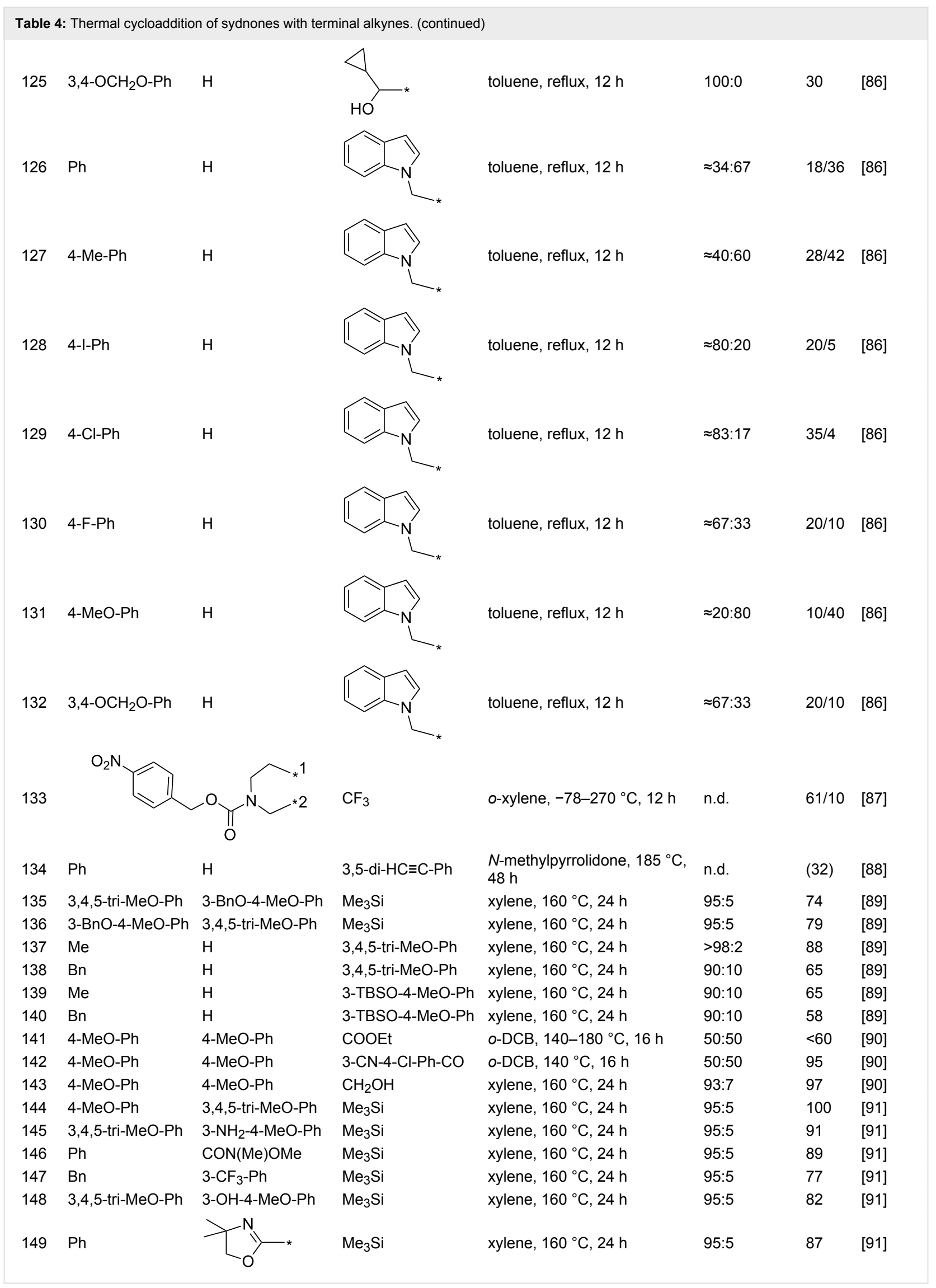




\begin{tabular}{|c|c|c|c|c|c|c|c|}
\hline 150 & 4-F-Ph & $\mathrm{H}$ & $\mathrm{Ph}$ & xylene, $160^{\circ} \mathrm{C}, 24 \mathrm{~h}$ & $90: 10$ & 100 & [91] \\
\hline 151 & 4-MeO-Ph & $\mathrm{H}$ & cyclo-Pr & xylene, $160^{\circ} \mathrm{C}, 24 \mathrm{~h}$ & $90: 10$ & 91 & [91] \\
\hline 152 & $\mathrm{Ph}$ & & cyclo-Pr & xylene, $160^{\circ} \mathrm{C}, 24 \mathrm{~h}$ & $95: 5$ & 91 & [91] \\
\hline 153 & $\mathrm{Ph}$ & 4-Me-Ph & $\mathrm{CH}_{2} \mathrm{OH}$ & xylene, $160^{\circ} \mathrm{C}, 24 \mathrm{~h}$ & $95: 5$ & 63 & [91] \\
\hline 154 & $\mathrm{Ph}$ & $\mathrm{CON}(\mathrm{Me}) \mathrm{OMe}$ & cyclo-Pr & xylene, $160^{\circ} \mathrm{C}, 24 \mathrm{~h}$ & $95: 5$ & 84 & [91] \\
\hline 155 & $\mathrm{Ph}$ & 2-Py & $\mathrm{Ph}$ & xylene, $160^{\circ} \mathrm{C}, 24 \mathrm{~h}$ & $95: 5$ & 98 & [91] \\
\hline 156 & $\mathrm{Ph}$ & $\mathrm{H}$ & COOEt & $o-D C B, 140^{\circ} \mathrm{C}, 16 \mathrm{~h}$ & $67: 33$ & 59 & [92] \\
\hline 156 & 4-MeO-Ph & $\mathrm{H}$ & COOEt & $o-D C B, 140{ }^{\circ} \mathrm{C}, 24 \mathrm{~h}$ & $67: 33$ & 57 & [92] \\
\hline
\end{tabular}

alsolated yield of single or both regioisomers. ${ }^{\mathrm{b}}$ In a sealed tube. n.d. - not determined.

regioisomers or at least chemical yield of the major regioisomer was given.

The first people who qualitatively discussed the regioselectivity on the basis of semi-empirical quantum calculations was Houk et al. [94] who (except of above-mentioned low-lying LUMO of sydnone [58]) calculated sydnone LUMO terminal orbital coefficients and found them to be almost identical thus indicating low selectivity in LUMO-controlled cycloadditions (type III). However, Gotthardt and Reiter [8] who were also dealing with regioselectivity of sydnone cycloadditions with methyl propiolate pointed out that the reason for the lower regioselectivity can also be attributed to the low-lying HOMO of this dipolarophile. While for the LUMO-controlled reaction (type III) only the 3 -substituted pyrazole is expected to be the main product, for the HOMO-controlled reaction (type I) 4-substituted pyrazole should be formed preferentially (Scheme 7 adapted from reference [8]).
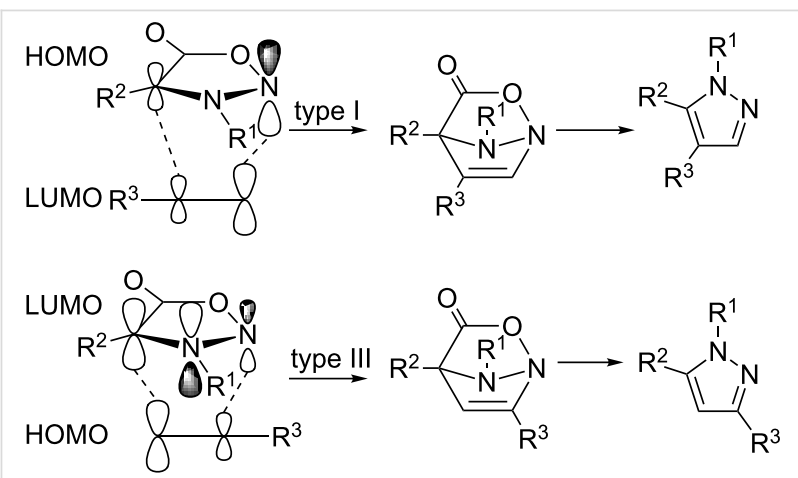

Scheme 7: HOMO-LUMO diagram for thermal [3 + 2]-cycloaddition of sydnones with alkynes.

The combination of both reaction pathways (type II) therefore gives a mixture of 3- and 4-substituted pyrazoles. This situation is typical especially for cycloadditions with alkyl propiolates (cf. Table 4, entries 6, 7, 23-29, 33, 34, 50-55, 58, 118, 141) and acylalkynes (Table 4 , entry 142). Other terminal alkynes, for which the calculated lower HOMO-LUMO energy gaps correspond to the type III mechanism (especially phenylacetylenes, alkylacetylenes, trimethylsilylacetylene and BPinacetylene), innately prefer formation of 1,3- (or 1,3,5-) di- (or tri-)substituted pyrazoles in ratios about or even better than 90:10. Recent quantum calculations undertaken for 3-phenylsydnone and phenylacetylene by Harrity et al. [92] clearly support a preferential formation of 1,3-diphenyl-1 $H$ pyrazole. The calculated difference in energies of transition states leading to 1,3- and 1,4-diphenyl- $1 H$-pyrazole $\left(4.1 \mathrm{kcal} \cdot \mathrm{mol}^{-1}=17.2 \mathrm{~kJ} \cdot \mathrm{mol}^{-1}\right)$ predicts at $140{ }^{\circ} \mathrm{C}$ the ratio $\approx 99: 1$ which corresponds well with found experimental value $>95: 5$ (see entry 3 in Table 4 [83]).

There are only some known exceptions in which the 1,4-substituted pyrazole prevails (Table 4, entries 8, 23, 31, 48, 126, 127 and 131). The most significant is the reaction of the parent phenylsydnone with phenylsulfonylacetylene which gives the ratio 25:75 [68] consistent with the strong electron-withdrawing effect of the phenylsulfonyl group lowering the HOMO of this dipolarophile. In addition, quantum calculations of orbital coefficients show that the HOMO is mainly located on the phenyl moiety and not in the acetylene moiety thus excluding the type III mechanism leading to a 1,3-disubstituted pyrazole.

Even though the HOMO-LUMO energy gaps and terminal orbital coefficients can be tuned by substitution of the sydnone (and alkyne) the ratio of both isomers is often only slightly influenced. For example 3-(substituted phenyl)sydnones react with methyl propiolate to give a mixture of both regioisomers in a 75:25 ratio (for 2,3-diMe [28], 4-OEt [75], H [1,2]), whilst only for $4-\mathrm{NO}_{2}$ derivative [8] is an enhanced ratio of $86: 14 \mathrm{ob}-$ served. The same pattern can be seen for reaction of 3,4diphenylsydnones. While unsubstituted $\left(\mathrm{R}^{1}=\mathrm{R}^{2}=\mathrm{Ph}\right)$ reacts with methyl propiolate to give equimolar amounts of the corresponding pyrazole 3-/4-carboxylate, an introduction of one or two nitro group(s) into position(s) 4- or 2,4- of the 3-phenyl 
ring $\left(\mathrm{R}^{1}=4-\mathrm{NO}_{2}-\mathrm{Ph}\right.$ or $\left.2,4-\mathrm{diNO}_{2}-\mathrm{Ph}\right)$ leads to ratios $56: 44$ and $61: 39$, respectively [8]. The presence of the nitro group(s) lower(s) the LUMO energy of the sydnone and a type III mechanism is slightly favored. The same trend [8] can be seen from the substitution effect in position 4 of the starting 3-phenylsydnone when reacted with methyl propiolate (Table 4, entries 6,7 , 23-26) but almost no influence is observed for reactions with phenylacetylene (Table 4, entries 3, 4, 69, 91). Generally, it can be concluded that any substituent in position 4 reduces the regioselectivity.

The steric hindrance can also affect the ratio of the regioisomers formed. The classical example was described by Yeh et al. [75,76] who performed reactions of 3-(4-ethoxyphenyl)sydnone-4-carbonitrile with various alkyl propiolates (Et, Bn, $t$ - $\mathrm{Bu}, \mathrm{Ph}_{2} \mathrm{CH}$ - see entries 52, 55-57 in Table 4) and observed that the 3-/4-ratio increased from 58:42 to 100:0. However, this trend is not general because Lee et al. [71] observed the best regioselectivity for the reaction of 4,5,6,7-tetrahydro[1,2,3]oxadiazolo[3,4- $a$ ]pyridin-8-ium-3-olate with methyl propiolate and the lowest selectivity with $n$-butyl and 1-phenylethyl propiolates (see entries $38-41$ in Table 4).

The last factor that influences the ratio of isomers involves the thermodynamic conditions - namely the temperature and pressure. A nice temperature/pressure-selectivity study of the cycloaddition of 3-phenylsydnone with methyl propiolate was undertaken by McGowin et al. [93] in supercritical $\mathrm{CO}_{2}$. At 7.6 $\mathrm{MPa}$ they found a linear dependence between the natural logarithm of selectivity (defined as the 3-/4-isomer ratio) and the reaction temperature. In accordance with the common reactivity-selectivity principle, the higher temperature lowers selectivity from 5.52 (i.e., $85: 15$ ) at $80{ }^{\circ} \mathrm{C}$ to 3.14 (i.e., 76:24) at $160{ }^{\circ} \mathrm{C}$ but increases sydnone conversion and pyrazole yield. On the other hand, a variation of the pressure from 7.6 to $30.4 \mathrm{MPa}$ at constant temperature $\left(80^{\circ} \mathrm{C}\right)$ caused a decrease in the total yield by approximately $50 \%$, with slightly increased selectivity (from 4.96 to 6.56). Lowering of the yield with increasing pressure confirms the reversibility of the first step (see Scheme 5) because of retardation of $\mathrm{CO}_{2}$ cleavage from the bicyclic intermediate. Such reversibility was also suggested by Harrity et al. [92] on the basis of quantum calculations. While the formation of the bicyclic intermediate was calculated to be only slightly exergonic $\left(-3.3 \mathrm{kcal} \cdot \mathrm{mol}^{-1}\right)$ the overall reaction is highly exothermic $\left(-108.2 \mathrm{kcal} \cdot \mathrm{mol}^{-1}\right)$.

These results show that minor differences in selectivity published by various authors (e.g., entry 3 in Table 4) can be ascribed to changes in temperature (different boiling point of benzene, toluene, xylenes, DCB , ...) used in synthesis. In several cases (e.g., entries 83-86 [84] and 141 and 142 [90] in
Table 4) too high temperature $\left(200^{\circ} \mathrm{C}\right)$ can contribute to a substantial drop of selectivity. It is also known that some sydnones start to decompose at temperatures exceeding $180{ }^{\circ} \mathrm{C}$ [74] which can cause lowering of the pyrazole yield.

From a synthetic point of view, the cycloaddition with terminal alkynes represents a very good strategy for the preparation of 1-,1,3-, 1,5- and 1,3,5-substituted pyrazoles. Although 1- and 1,5-(di)substituted pyrazoles are directly available from 3- or 3,4-(di)substituted sydnones and acetylene (e.g., entry 1 in Table $1[1,2])$, handling with gaseous acetylene or its solution in pressurized reaction vessels is inconvenient and may be even dangerous. Two strategies can overcome such problems: liquid DMAD, diethyl acetylenedicarboxylate or alkyl propiolate can be used instead of acetylene and the resulting pyrazole-3,4dicarboxylates or pyrazole-3-/4-carboxylates can then undergo hydrolysis and decarboxylation $[16,95,96]$. A novel strategy (Scheme 8) was recently developed by Harrity et al. (see entries $73,74,94,117,136,137,145-150$ [32-34,82,89,91] in Table 4) who used trimethylsilyl acetylene as a dipolarophile. After regioselective cycloaddition giving 3-trimethylsilylpyrazole (cf. also entries 59-61 [78]) in high yields (74-100\%) the trimethylsilyl group was removed by TBAF-mediated protodesilylation in moderate to good yields (47-76\%).

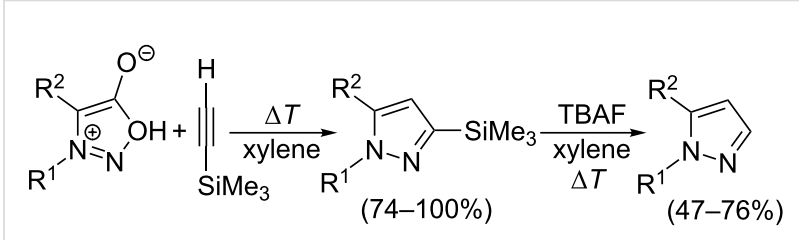

$\mathrm{R}^{1}$ : Ph, subst.Ph, Bn $\mathrm{R}^{2}: \mathrm{H}, \mathrm{I}, \mathrm{CF}_{3}$, subst. Ph, heterocyclyl

Scheme 8: Synthetic strategy leading to 1,2-disubstituted pyrazoles.

\section{Thermal reaction of sydnones with internal non-symmetrical alkynes and cycloakynes}

The reaction with internal non-symmetrical alkynes giving 1,3,4-trisubstituted or even 1,3,4,5-tetrasubstituted pyrazoles seems to be the most complicated case of cycloaddition due to the influence of both substituents $\left(\mathrm{R}^{3}, \mathrm{R}^{4}\right)$ bound on a triple bond on the formation of pyrazole regioisomers. Moreover, not all substituents are compatible with the reaction conditions. For example phenylpropiolic acid $\left(\mathrm{R}^{3}: \mathrm{COOH}\right)$ gives only minor a yield of the cycloaddition/decarboxylation product with 5,6dihydro-3-hydroxy-4H-pyrrolo[1,2-c][1,2,3]oxadiazol-7-ium [97,98] (Scheme 9).

The Table 5 again summarizes all the examples found, including reaction conditions from which we have come to several conclusions. 


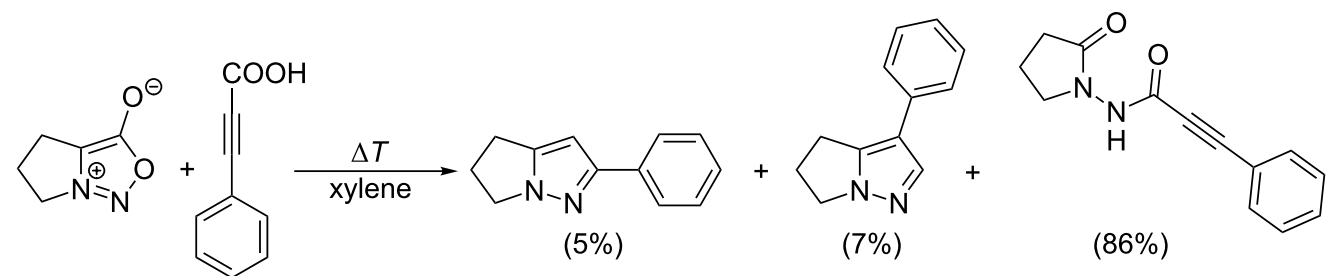

Scheme 9: Unsuccessful reaction with phenylpropiolic acid.

Table 5: Thermal cycloaddition of sydnones with internal non-symmetrical alkynes.

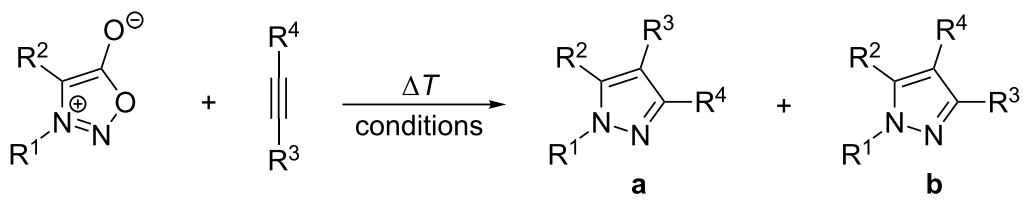

\begin{tabular}{|c|c|c|c|c|c|c|c|c|}
\hline entry & $\mathrm{R}^{1}$ & $\mathrm{R}^{2}$ & $\mathrm{R}^{3}$ & $\mathrm{R}^{4}$ & conditions & $\begin{array}{l}\text { ratio } \\
a: b\end{array}$ & $\begin{array}{l}\text { yield } \\
{[\%]^{\mathrm{a}}}\end{array}$ & ref. \\
\hline 1 & $\mathrm{Ph}$ & $\mathrm{H}$ & $\mathrm{Me}$ & $\mathrm{Ph}$ & xylene, $135-140^{\circ} \mathrm{C}, 20 \mathrm{~h}$ & n.d. & $83 /-$ & {$[1,2]$} \\
\hline 2 & $\mathrm{Ph}$ & $\mathrm{H}$ & COOEt & $\mathrm{Ph}$ & toluene, $95^{\circ} \mathrm{C}, 84 \mathrm{~h}$ & n.d. & 82-83/- & {$[1,2]$} \\
\hline 3 & 4-Cl-Ph & $\mathrm{H}$ & COOEt & $\mathrm{Ph}$ & xylene, reflux, $3 \mathrm{~h}$ & n.d. & $92 /-$ & [2] \\
\hline 4 & 4-MeO-Ph & $\mathrm{H}$ & COOEt & $\mathrm{Ph}$ & xylene, reflux, $3 \mathrm{~h}$ & n.d. & $83 /-$ & [2] \\
\hline 5 & 4-Me-Ph & $\mathrm{H}$ & COOEt & $\mathrm{Ph}$ & xylene, reflux, $3 \mathrm{~h}$ & n.d. & $98 /-$ & [2] \\
\hline 6 & $\mathrm{Bn}$ & $\mathrm{H}$ & COOEt & $\mathrm{Ph}$ & xylene, reflux, $16 \mathrm{~h}$ & n.d. & $46 /-$ & [2] \\
\hline 7 & $\mathrm{Ph}$ & $\mathrm{Ph}$ & COOEt & $\mathrm{Ph}$ & p-cymene, $160^{\circ} \mathrm{C}, 16 \mathrm{~h}$ & n.d. & $87 /-$ & [2] \\
\hline 8 & $\mathrm{Ph}$ & $\mathrm{Me}$ & COOEt & $\mathrm{Ph}$ & xylene, $110^{\circ} \mathrm{C}, 8 \mathrm{~h}$ & $100: 0$ & 82 & {$[1,2]$} \\
\hline 9 & $\mathrm{Ph}$ & $\mathrm{H}$ & COMe & $\mathrm{Ph}$ & chlorobenzene, $130^{\circ} \mathrm{C}, 12 \mathrm{~h}$ & 100:0 & 100 & {$[1,2]$} \\
\hline 10 & $\mathrm{Ph}$ & $\mathrm{H}$ & $\mathrm{COPh}$ & $\mathrm{Ph}$ & xylene, $135-140^{\circ} \mathrm{C}, 16 \mathrm{~h}$ & 100:0 & 82 & {$[1,2]$} \\
\hline 11 & $\mathrm{Me}$ & $\mathrm{H}$ & $\mathrm{COPh}$ & $\mathrm{Ph}$ & $o-D C B$, reflux, $144 \mathrm{~h}$ & $69: 31$ & 99 & [16] \\
\hline 12 & $\mathrm{Ph}$ & $\mathrm{H}$ & $\mathrm{CN}$ & $\mathrm{Cl}$ & chlorobenzene, $110^{\circ} \mathrm{C}, 10 \mathrm{~h}$ & n.d. & $15 / 20$ & [66] \\
\hline 13 & $\mathrm{Ph}$ & $\mathrm{H}$ & $\mathrm{SO}_{2} \mathrm{Ph}$ & $\mathrm{Me}$ & toluene, $100^{\circ} \mathrm{C}, 24 \mathrm{~h}$ & $100: 0$ & 58 & [68] \\
\hline 14 & $\mathrm{Ph}$ & $\mathrm{H}$ & $\mathrm{SO}_{2} \mathrm{Ph}$ & $\mathrm{Ph}$ & toluene, $100^{\circ} \mathrm{C}, 24 \mathrm{~h}$ & $100: 0$ & 73 & [68] \\
\hline 15 & $\mathrm{Ph}$ & $\mathrm{H}$ & COOMe & $\mathrm{CH}(\mathrm{OMe})_{2}$ & toluene, reflux, $60 \mathrm{~h}$ & $21: 79$ & 84 & [99] \\
\hline 16 & $\mathrm{Bn}$ & $\mathrm{H}$ & COOMe & $\mathrm{CH}(\mathrm{OMe})_{2}$ & toluene, reflux, $72 \mathrm{~h}$ & $19: 81$ & 80 & [99] \\
\hline 17 & $\mathrm{Bn}$ & $\mathrm{H}$ & coOMe & $\mathrm{CHO}$ & toluene, reflux, $18 \mathrm{~h}$ & $72: 28$ & 90 & [99] \\
\hline 18 & $\mathrm{Ph}$ & $\mathrm{H}$ & COOMe & $\mathrm{CHO}$ & toluene, reflux, $18 \mathrm{~h}$ & $66: 34$ & 93 & [99] \\
\hline 19 & $\mathrm{Bn}$ & $\mathrm{H}$ & coOMe & $\mathrm{CH}_{2} \mathrm{OH}$ & toluene, reflux, $72 \mathrm{~h}$ & $50: 50$ & 75 & [99] \\
\hline 20 & $\mathrm{Ph}$ & $\mathrm{H}$ & coOMe & $\mathrm{CH}_{2} \mathrm{OH}$ & toluene, reflux, $48 \mathrm{~h}$ & $60: 40$ & 79 & [99] \\
\hline 21 & $\mathrm{Ph}$ & $\mathrm{H}$ & $\mathrm{CF}_{3}$ & 4-MeO-Ph & xylene, $120^{\circ} \mathrm{C}, 48-72 \mathrm{~h}$ & $93: 7$ & 56 & [100] \\
\hline 22 & $\mathrm{Ph}$ & $\mathrm{H}$ & $\mathrm{CF}_{3}$ & $4-\mathrm{NO}_{2}-\mathrm{Ph}$ & xylene, $120^{\circ} \mathrm{C}, 48-72 \mathrm{~h}$ & $93: 7$ & 93 & [100] \\
\hline 23 & $\mathrm{Ph}$ & $\mathrm{H}$ & $\mathrm{CF}_{3}$ & 4-MeS-Ph & xylene, $120^{\circ} \mathrm{C}, 48-72 \mathrm{~h}$ & $93: 7$ & 90 & [100] \\
\hline 24 & $\mathrm{Ph}$ & $\mathrm{H}$ & $\mathrm{CF}_{3}$ & 2-Cl-Ph & xylene, $120^{\circ} \mathrm{C}, 48-72 \mathrm{~h}$ & $94: 6$ & 92 & [100] \\
\hline 25 & $\mathrm{Ph}$ & $\mathrm{H}$ & $\mathrm{CF}_{3}$ & $4-\mathrm{MeSO}_{2}-\mathrm{Ph}$ & xylene, $120^{\circ} \mathrm{C}, 48-72 \mathrm{~h}$ & $92: 8$ & 86 & [100] \\
\hline 26 & $\mathrm{Ph}$ & $\mathrm{H}$ & $\mathrm{CF}_{3}$ & 4-Cl-Ph & xylene, $120^{\circ} \mathrm{C}, 48-72 \mathrm{~h}$ & $93: 7$ & 75 & [100] \\
\hline 27 & 4-Cl-Ph & $\mathrm{H}$ & $\mathrm{CF}_{3}$ & 4-Cl-Ph & xylene, $120^{\circ} \mathrm{C}, 48-72 \mathrm{~h}$ & $93: 7$ & 90 & [100] \\
\hline 28 & 4-MeO-Ph & $\mathrm{H}$ & $\mathrm{CF}_{3}$ & 4-Cl-Ph & xylene, $120^{\circ} \mathrm{C}, 48-72 \mathrm{~h}$ & $93: 7$ & 84 & [100] \\
\hline 29 & $\mathrm{Bn}$ & $\mathrm{H}$ & $\mathrm{CF}_{3}$ & 4-Cl-Ph & xylene, $120^{\circ} \mathrm{C}, 48-72 \mathrm{~h}$ & $91: 9$ & 65 & [100] \\
\hline 30 & $t-\mathrm{Bu}$ & $\mathrm{H}$ & $\mathrm{CF}_{3}$ & 4-Cl-Ph & xylene, $120^{\circ} \mathrm{C}, 48-72 \mathrm{~h}$ & $93: 7$ & 58 & [100] \\
\hline 31 & $\mathrm{Me}$ & $\mathrm{H}$ & $\mathrm{CF}_{3}$ & 4-Cl-Ph & xylene, $120^{\circ} \mathrm{C}, 48-72 \mathrm{~h}$ & $92: 8$ & 92 & [100] \\
\hline 32 & $\mathrm{Ph}$ & $\mathrm{Me}$ & $\mathrm{CF}_{3}$ & 4-Cl-Ph & xylene, $120^{\circ} \mathrm{C}, 48-72 \mathrm{~h}$ & $84: 16$ & 75 & [100] \\
\hline 33 & $\mathrm{Ph}$ & 4-Cl-Ph & $\mathrm{CF}_{3}$ & 4-Cl-Ph & xylene, $120^{\circ} \mathrm{C}, 48-72 \mathrm{~h}$ & $60: 40$ & 57 & [100] \\
\hline 34 & $\mathrm{Ph}$ & $\mathrm{Br}$ & $\mathrm{CF}_{3}$ & 4-Cl-Ph & xylene, $120^{\circ} \mathrm{C}, 48-72 \mathrm{~h}$ & $71: 29$ & 73 & [100] \\
\hline
\end{tabular}




\begin{tabular}{|c|c|c|c|c|c|c|c|c|}
\hline 35 & $\mathrm{Ph}$ & MeS & $\mathrm{CF}_{3}$ & 4-Cl-Ph & xylene, $120^{\circ} \mathrm{C}, 48-72 \mathrm{~h}$ & $43: 57$ & 62 & [100] \\
\hline 36 & $t-\mathrm{Bu}$ & $\mathrm{H}$ & COOEt & $\mathrm{Et}$ & xylene, reflux, $72 \mathrm{~h}$ & n.d. & $38 / 8$ & [101] \\
\hline 37 & $\mathrm{Ph}$ & $\mathrm{H}$ & $\mathrm{PhCO}$ & $5-\mathrm{NO}_{2}$-furan-2-yl & xylene, reflux, 3-4 h & $100: 0$ & 74 & [102] \\
\hline 38 & $\mathrm{Ph}$ & $\mathrm{H}$ & 4-Me-PhCO & $5-\mathrm{NO}_{2}$-furan-2-yl & xylene, reflux, 3-4 h & $100: 0$ & 80 & [102] \\
\hline 39 & $\mathrm{Ph}$ & $\mathrm{H}$ & 4-Cl-PhCO & $5-\mathrm{NO}_{2}$-furan-2-yl & xylene, reflux, 3-4 h & $100: 0$ & 72 & [102] \\
\hline 40 & 4-MeO-Ph & $\mathrm{H}$ & $\mathrm{PhCO}$ & $5-\mathrm{NO}_{2}$-furan-2-yl & xylene, reflux, 3-4 h & $100: 0$ & 73 & [102] \\
\hline 41 & 4-MeO-Ph & $\mathrm{H}$ & 4-Me-PhCO & $5-\mathrm{NO}_{2}$-furan-2-yl & xylene, reflux, 3-4 h & $100: 0$ & 74 & [102] \\
\hline 42 & 4-MeO-Ph & $\mathrm{H}$ & 4-Cl-PhCO & $5-\mathrm{NO}_{2}$-furan-2-yl & xylene, reflux, 3-4 h & $100: 0$ & 73 & [102] \\
\hline 43 & 4-Me-Ph & $\mathrm{H}$ & $\mathrm{PhCO}$ & $5-\mathrm{NO}_{2}$-furan-2-yl & xylene, reflux, 3-4 h & $100: 0$ & 79 & [102] \\
\hline 44 & 4-Me-Ph & $\mathrm{H}$ & 4-Me-PhCO & $5-\mathrm{NO}_{2}$-furan-2-yl & xylene, reflux, 3-4 h & $100: 0$ & 83 & [102] \\
\hline 45 & 4-Me-Ph & $\mathrm{H}$ & 4-Cl-PhCO & $5-\mathrm{NO}_{2}$-furan-2-yl & xylene, reflux, 3-4 h & $100: 0$ & 75 & [102] \\
\hline 46 & $\mathrm{Me}$ & $\mathrm{H}$ & COOEt & $\mathrm{CF}_{3}$ & xylene, $100^{\circ} \mathrm{C}, 4 \mathrm{~h}$ & n.d. & $18 / 25$ & [103] \\
\hline 47 & $\mathrm{Ph}$ & $\mathrm{H}$ & $\mathrm{SnBu}_{3}$ & $\mathrm{SiMe}_{3}$ & toluene, reflux & $100: 0$ & 80 & [78] \\
\hline 48 & $\mathrm{Ph}$ & $\mathrm{H}$ & $\mathrm{SiMe}_{2} \mathrm{Ph}$ & $\mathrm{SiMe}_{3}$ & toluene, reflux & n.d. & $63 / 34$ & [78] \\
\hline 49 & $\mathrm{Ph}$ & $\mathrm{H}$ & COMe & $\mathrm{SiMe}_{3}$ & toluene, reflux & n.d. & $81 / 16$ & [78] \\
\hline 50 & $\mathrm{Ph}$ & $\mathrm{H}$ & BPin & $\mathrm{Ph}$ & xylene, reflux, $4 \mathrm{~h}$ & $98: 2$ & 58 & {$[79,81]$} \\
\hline 51 & $\mathrm{Ph}$ & $\mathrm{H}$ & BPin & $\mathrm{Bu}$ & xylene, reflux, $4 \mathrm{~h}$ & $71: 29$ & 64 & {$[79,81]$} \\
\hline 52 & $\mathrm{Ph}$ & $\mathrm{H}$ & BPin & $\mathrm{Me}_{3} \mathrm{Si}$ & xylene, reflux, $4 \mathrm{~h}$ & $67: 33$ & 76 & {$[79,81]$} \\
\hline 53 & 4-MeO-Ph & $\mathrm{H}$ & BPin & $\mathrm{Ph}$ & xylene, reflux, $4 \mathrm{~h}$ & $98: 2$ & 58 & [79] \\
\hline 54 & $4-\mathrm{NO}_{2}-\mathrm{Ph}$ & $\mathrm{H}$ & BPin & $\mathrm{Ph}$ & xylene, reflux, $4 \mathrm{~h}$ & $98: 2$ & 70 & [79] \\
\hline 55 & 4-MeO-Ph & $\mathrm{H}$ & BPin & $\mathrm{Bu}$ & xylene, reflux, $4 \mathrm{~h}$ & $83: 17$ & 55 & [79] \\
\hline 56 & $4-\mathrm{NO}_{2}-\mathrm{Ph}$ & $\mathrm{H}$ & BPin & $\mathrm{Bu}$ & xylene, reflux, $4 \mathrm{~h}$ & $83: 17$ & 62 & [79] \\
\hline 57 & 4-MeO-Ph & $\mathrm{H}$ & BPin & $\mathrm{Me}_{3} \mathrm{Si}$ & xylene, reflux, $4 \mathrm{~h}$ & $67: 33$ & 61 & [79] \\
\hline 58 & $4-\mathrm{NO}_{2}-\mathrm{Ph}$ & $\mathrm{H}$ & BPin & $\mathrm{Me}_{3} \mathrm{Si}$ & xylene, reflux, $4 \mathrm{~h}$ & $60: 40$ & 83 & [79] \\
\hline 59 & 3-Py & $\mathrm{H}$ & BPin & $\mathrm{Ph}$ & xylene, reflux, $16 \mathrm{~h}$ & $>98: 2$ & 60 & [84] \\
\hline 60 & 3-Py & $\mathrm{H}$ & BPin & $\mathrm{Me}_{3} \mathrm{Si}$ & xylene, reflux, $16 \mathrm{~h}$ & $57: 43$ & 70 & {$[81,84]$} \\
\hline 61 & 3-Py & $\mathrm{H}$ & BPin & $n$-Bu & mesitylene, reflux, $16 \mathrm{~h}$ & $71: 29$ & 56 & [84] \\
\hline 62 & 4-Me-Ph & $\mathrm{CHO}$ & $\mathrm{PhCO}$ & $5-\mathrm{NO}_{2}$-furan-2-yl & xylene, reflux, 3-4 h & n.d. & $79 /-$ & [104] \\
\hline 63 & 4-Me-Ph & $\mathrm{CHO}$ & 4-Me-PhCO & $5-\mathrm{NO}_{2}$-furan-2-yl & xylene, reflux, 3-4 h & n.d. & $74 /-$ & [104] \\
\hline 64 & 4-Me-Ph & $\mathrm{Br}$ & 4-MeO-PhCO & $5-\mathrm{NO}_{2}$-furan-2-yl & xylene, reflux, 3-4 h & n.d. & $62 /-$ & [104] \\
\hline 65 & 4-MeO-Ph & $\mathrm{Br}$ & 4-MeO-PhCO & $5-\mathrm{NO}_{2}$-furan-2-yl & xylene, reflux, 3-4 h & n.d. & $69 /-$ & [104] \\
\hline 66 & 4-Me-Ph & $\mathrm{Br}$ & $\mathrm{PhCO}$ & $5-\mathrm{NO}_{2}$-furan-2-yl & xylene, reflux, 3-4 h & n.d. & $73 /-$ & [104] \\
\hline 67 & 4-Me-Ph & $\mathrm{Br}$ & 4-Me-PhCO & $5-\mathrm{NO}_{2}$-furan-2-yl & xylene, reflux, 3-4 h & n.d. & $66 /-$ & [104] \\
\hline 68 & 4-MeO-Ph & $\mathrm{Br}$ & 4-Me-PhCO & $5-\mathrm{NO}_{2}$-furan-2-yl & xylene, reflux, 3-4 h & n.d. & $63 /-$ & [104] \\
\hline 69 & $\mathrm{Ph}$ & $\mathrm{Br}$ & 4-Me-PhCO & $5-\mathrm{NO}_{2}$-furan-2-yl & xylene, reflux, 3-4 h & n.d. & $72 /-$ & [104] \\
\hline 70 & 4-MeO-Ph & $\mathrm{MeCO}$ & $\mathrm{PhCO}$ & $5-\mathrm{NO}_{2}$-furan-2-yl & xylene, reflux, 3-4 h & n.d. & $74 /-$ & [104] \\
\hline 71 & 4-MeO-Ph & $\mathrm{MeCO}$ & 4-MeO-PhCO & 5- $\mathrm{NO}_{2}$-furan-2-yl & xylene, reflux, 3-4 h & n.d. & $73 /-$ & [104] \\
\hline 72 & $\mathrm{Ph}$ & $\mathrm{H}$ & 4-Me-PhCO & 5- $\mathrm{NO}_{2}$-thiophen-2-yl & xylene, reflux, 3-4 h & n.d. & $71 /-$ & [105] \\
\hline 73 & $\mathrm{Ph}$ & $\mathrm{H}$ & 4-MeO-PhCO & 5- $\mathrm{NO}_{2}$-thiophen-2-yl & xylene, reflux, 3-4 h & n.d. & 73/- & [105] \\
\hline 74 & 4-Me-Ph & $\mathrm{H}$ & $\mathrm{PhCO}$ & 5- $\mathrm{NO}_{2}$-thiophen-2-yl & xylene, reflux, 3-4 h & n.d. & $75 /-$ & [105] \\
\hline 75 & 4-Me-Ph & $\mathrm{H}$ & 4-Me-PhCO & 5- $\mathrm{NO}_{2}$-thiophen-2-yl & xylene, reflux, 3-4 h & n.d. & $73 /-$ & [105] \\
\hline 76 & $\mathrm{Ph}$ & $\mathrm{H}$ & 4-Cl-PhCO & 5- $\mathrm{NO}_{2}$-thiophen-2-yl & xylene, reflux, 3-4 h & n.d. & $72 /-$ & [105] \\
\hline 77 & 4-Me-Ph & $\mathrm{H}$ & 4-Cl-PhCO & 5- $\mathrm{NO}_{2}$-thiophen-2-yl & xylene, reflux, 3-4 h & n.d. & $77 /-$ & [105] \\
\hline 78 & 4-MeO-Ph & $\mathrm{H}$ & 4-Cl-PhCO & 5- $\mathrm{NO}_{2}$-thiophen-2-yl & xylene, reflux, 3-4 h & n.d. & $78 /-$ & [105] \\
\hline 79 & $\mathrm{Ph}$ & $\mathrm{H}$ & $\mathrm{PhCO}$ & 5- $\mathrm{NO}_{2}$-thiophen-2-yl & xylene, reflux, 3-4 h & n.d. & $80 /-$ & [105] \\
\hline 80 & 4-MeO-Ph & $\mathrm{H}$ & $\mathrm{PhCO}$ & 5- $\mathrm{NO}_{2}$-thiophen-2-yl & xylene, reflux, 3-4 h & n.d. & $75 /-$ & [105] \\
\hline 81 & 4-MeO-Ph & $\mathrm{H}$ & 4-Me-PhCO & 5- $\mathrm{NO}_{2}$-thiophen-2-yl & xylene, reflux, 3-4 h & n.d. & $76 /-$ & [105] \\
\hline 82 & \multicolumn{2}{|c|}{$\mathrm{CH}_{2} \mathrm{CH}_{2} \mathrm{CH}_{2}$} & $p$-Tos & $\mathrm{Bu}$ & anisol, reflux, $0.5 \mathrm{~h}$ & n.d. & $90 /-$ & [106] \\
\hline 83 & \multicolumn{2}{|c|}{$\mathrm{CH}_{2} \mathrm{CH}_{2} \mathrm{CH}_{2}$} & $p$-Tos & $\mathrm{Ph}$ & anisol, reflux, $0.5 \mathrm{~h}$ & n.d. & $89 /-$ & [106] \\
\hline 84 & $\mathrm{Ph}$ & 4-Me-Ph & BPin & $\mathrm{Me}_{3} \mathrm{Si}$ & o-DCB, reflux, $48 \mathrm{~h}$ & $100: 0$ & 48 & [80] \\
\hline 85 & $\mathrm{Ph}$ & $4-\mathrm{NO}_{2}-\mathrm{Ph}$ & BPin & $\mathrm{Me}_{3} \mathrm{Si}$ & $o-D C B$, reflux, $48 \mathrm{~h}$ & $100: 0$ & 70 & [80] \\
\hline 86 & $\mathrm{Me}$ & $\mathrm{H}$ & BPin & $\mathrm{Ph}$ & mesitylene, reflux, $48 \mathrm{~h}$ & $>98: 2$ & 53 & [81] \\
\hline 87 & $\mathrm{Bn}$ & $\mathrm{H}$ & BPin & $\mathrm{Ph}$ & xylene, reflux & $>98: 2$ & 62 & [81] \\
\hline 88 & $\mathrm{Ph}$ & $\mathrm{Ph}$ & BPin & $\mathrm{Ph}$ & $o-D C B$, reflux, $48 \mathrm{~h}$ & $>98: 2$ & 59 & [81] \\
\hline
\end{tabular}




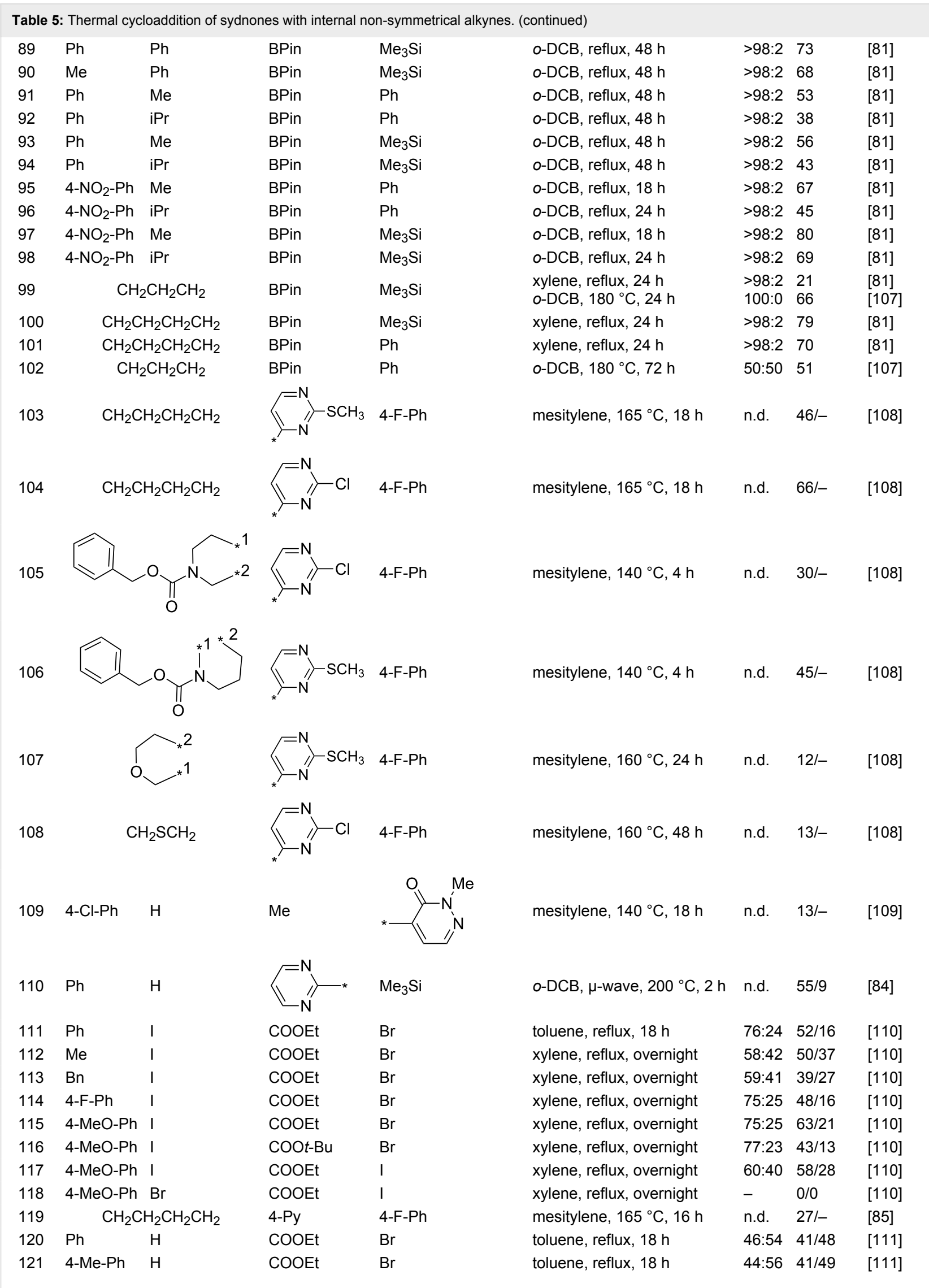




\begin{tabular}{|c|c|c|c|c|c|c|c|c|}
\hline 122 & 4-MeO-Ph & $\mathrm{H}$ & COOEt & $\mathrm{Br}$ & toluene, reflux, $18 \mathrm{~h}$ & 41:59 & $33 / 49$ & [111] \\
\hline 123 & $4-F-P h$ & $\mathrm{H}$ & COOEt & $\mathrm{Br}$ & toluene, reflux, $18 \mathrm{~h}$ & $47: 53$ & $38 / 43$ & [111] \\
\hline 124 & $\mathrm{Ph}$ & $\mathrm{CF}_{3}$ & coOMe & $\mathrm{Me}$ & $\mathrm{o}-\mathrm{DCB}, 180^{\circ} \mathrm{C}, 24 \mathrm{~h}$ & $85: 15$ & 90 & [32] \\
\hline 125 & $\mathrm{Ph}$ & $\mathrm{CF}_{3}$ & $\mathrm{Ph}$ & $n-\mathrm{Bu}$ & $\mathrm{o}-\mathrm{DCB}, 180^{\circ} \mathrm{C}, 24 \mathrm{~h}$ & $52: 48$ & 62 & [32] \\
\hline 126 & $\mathrm{Ph}$ & $\mathrm{CF}_{3}$ & BPin & $\mathrm{Me}_{3} \mathrm{Si}$ & $\mathrm{o}-\mathrm{DCB}, 140^{\circ} \mathrm{C}, 48 \mathrm{~h}$ & $90: 10$ & 68 & [32] \\
\hline 127 & $\mathrm{Ph}$ & $\mathrm{CF}_{3}$ & BPin & $n-\mathrm{Bu}$ & o-DCB, $140^{\circ} \mathrm{C}, 72 \mathrm{~h}$ & $>98: 2$ & 55 & [32] \\
\hline 128 & $\mathrm{Me}$ & $\begin{array}{l}3,4,5- \\
\text { triMeO-Ph }\end{array}$ & BPin & $\mathrm{Me}_{3} \mathrm{Si}$ & xylene, $180^{\circ} \mathrm{C}, 24 \mathrm{~h}$ & $83: 17$ & 92 & [89] \\
\hline 129 & $\mathrm{Bn}$ & $\begin{array}{l}3,4,5- \\
\text { triMeO-Ph }\end{array}$ & BPin & $\mathrm{Me}_{3} \mathrm{Si}$ & xylene, $180^{\circ} \mathrm{C}, 24 \mathrm{~h}$ & $90: 10$ & 66 & [89] \\
\hline 130 & $\mathrm{Me}$ & $\begin{array}{l}\text { 3-BnO- } \\
4-\mathrm{MeO}-\mathrm{Ph}\end{array}$ & BPin & $\mathrm{Me}_{3} \mathrm{Si}$ & xylene, $180^{\circ} \mathrm{C}, 24 \mathrm{~h}$ & $80: 20$ & 73 & [89] \\
\hline 131 & $\mathrm{Bn}$ & $\begin{array}{l}\text { 3-BnO- } \\
\text { 4-MeO-Ph }\end{array}$ & BPin & $\mathrm{Me}_{3} \mathrm{Si}$ & xylene, $180^{\circ} \mathrm{C}, 24 \mathrm{~h}$ & $90: 10$ & 64 & [89] \\
\hline 132 & $\mathrm{Ph}$ & 4-Me-Ph & BPin & $\mathrm{Me}_{3} \mathrm{Si}$ & xylene, $180^{\circ} \mathrm{C}, 48 \mathrm{~h}$ & $>98: 2$ & 74 & [91] \\
\hline 133 & $\mathrm{Ph}$ & 2-Py & BPin & $\mathrm{Me}_{3} \mathrm{Si}$ & xylene, $180^{\circ} \mathrm{C}, 48 \mathrm{~h}$ & $>98: 2$ & 52 & [91] \\
\hline 134 & $\mathrm{Ph}$ & 2-thienyl & BPin & $\mathrm{Me}_{3} \mathrm{Si}$ & xylene, $180^{\circ} \mathrm{C}, 48 \mathrm{~h}$ & $88: 12$ & $64 / 6$ & [91] \\
\hline 135 & $\mathrm{Me}$ & 4-Me-Ph & BPin & $\mathrm{Me}_{3} \mathrm{Si}$ & xylene, $180^{\circ} \mathrm{C}, 48 \mathrm{~h}$ & $>98: 2$ & 55 & [91] \\
\hline 136 & 4-EtO-Ph & 4-MeO-Ph & BPin & $\mathrm{Me}_{3} \mathrm{Si}$ & xylene, $180^{\circ} \mathrm{C}, 48 \mathrm{~h}$ & $88: 12$ & 74 & [91] \\
\hline
\end{tabular}

asolated overall yield or isolated yields of both regioisomers a/b. n.d. - not determined.

According to frontier molecular orbital theory both combinations, i.e., HOMO(dipole)-LUMO(dipolarophile) (type I) and HOMO(dipolarophile)-LUMO(dipole) (type III) should lead to the production of individual regioisomers (cf. Scheme 7). All substituents $\mathrm{R}^{1}-\mathrm{R}^{4}$ have an influence on the HOMO-LUMO energy gaps and consequently on the ratio of both isomers especially in those cases when such energy gaps are similar. Again, the substituents on the alkyne $\left(\mathrm{R}^{3}, \mathrm{R}^{4}\right)$ have great influence on the outcome of the reactions. Strong electron-withdrawing substituents $\mathrm{R}^{3}$ (COOR, COR, $\mathrm{SO}_{2} \mathrm{Ar}, \mathrm{CF}_{3}$ ) in combination with any aryl $\left(\mathrm{R}^{4}: \mathrm{Ph}\right.$, substituted $\mathrm{Ph}$, heteroaryls) strongly prefer position 4 in the final pyrazole ring (see entries 2-6, 9, 10, 14 $21-28,37-45,64-83$ in Table 5) when reacting with 4-unsubstituted 3-phenylsydnones or 3-alkylsydnones (see entries 6, 29-31 in Table 5). Both these substituents jointly lower the LUMO while their influence on energy of the HOMO is contradictory. Consequently, the type I mechanism is clearly preferred. If $\mathrm{R}^{4}$ has also similar electron-withdrawing ability (e.g., $\mathrm{CHO}, \mathrm{CF}_{3}$, see entries $17,18,46$ in Table 5 or $\mathrm{R}^{4}=$ halogen, see entries $12,120-123$ in Table 5 and even $\mathrm{R}^{4}=\mathrm{CH}_{2} \mathrm{OH}$, see entries 19 and 20 in Table 5) then almost complete loss of selectivity occurs and the ratio of both regioisomers is close to 50:50. Markedly reversed regioselectivity is observed only for $\mathrm{R}^{4}=\mathrm{CH}(\mathrm{OMe})_{2}$ which is probably connected with the higher steric demands of this group.

A substitution in position 3 of the sydnone has a much smaller influence on the regioselectivity which is in accordance with longer distance between the substituent and both dipole termini. Substitution of the 3-phenyl ring (e.g., entries 26-28, 37-45, 50,
53, 54 in Table 5) or even change of the whole 3-substituent (alkyls vs phenyl, see entries 29-31, 86, 87 in Table 5) cause no or only a minor change in the ratio of both regioisomers. In some cases the same conclusion can be drawn for changes of the substituent in 4-position of the sydnone (cf. entries 2, 7, 8, $89,93,94$ or 88, 91, 92 in Table 5). On the other hand, the presence of a substituent can sometimes increase as well as decrease the ratio of both regioisomers (cf. entries 26 and 32-35) for no easily discernible reason.

A synthetically useful cycloaddition of 4-substituted 3-phenylsydnones with 4,4,5,5-tetramethyl-2-(2-substituted ethynyl)1,3,2-dioxaborolanes $\left(\mathrm{R}^{4}-\mathrm{C} \equiv \mathrm{C}\right.$-BPin; Scheme 10$)$ was recently developed by Harrity et al. [79-81,84,91,107]. In most cases this reaction proceeds with excellent regioselectivity $(>98: 2)$ to give the corresponding 1-(substituted phenyl)-3,5-disubstituted -4-BPin-pyrazole, whose BPin group can be easily substituted by an aryl group using a Suzuki-Miyaura cross-coupling reaction. In those cases when a trimethylsilyl group $\left(\mathrm{R}^{4}\right)$ is also present, it can be removed by TBAF-mediated protodesilylation to give a 1,4,5-trisubstituted pyrazole.

It is worth noting that the parent 4,4,5,5-tetramethyl-2-ethynyl1,3,2-dioxaborolane $\left(\mathrm{R}^{4}: \mathrm{H}\right)$ reacts with alkyl/arylsydnones with completely opposite ratio ( $>7: 1)$ of both regioisomers - i.e., for the BPin group the reaction preferentially occurs in position 3 of the final pyrazole (see entries 62,64-66, 89, 109 and 110 in Table 4). In this case, using quantum chemical calculations (DFT/B3LYP-6-31G*) [81] steric effects were identified as the main factor influencing the ratio of both regioisomers. These 


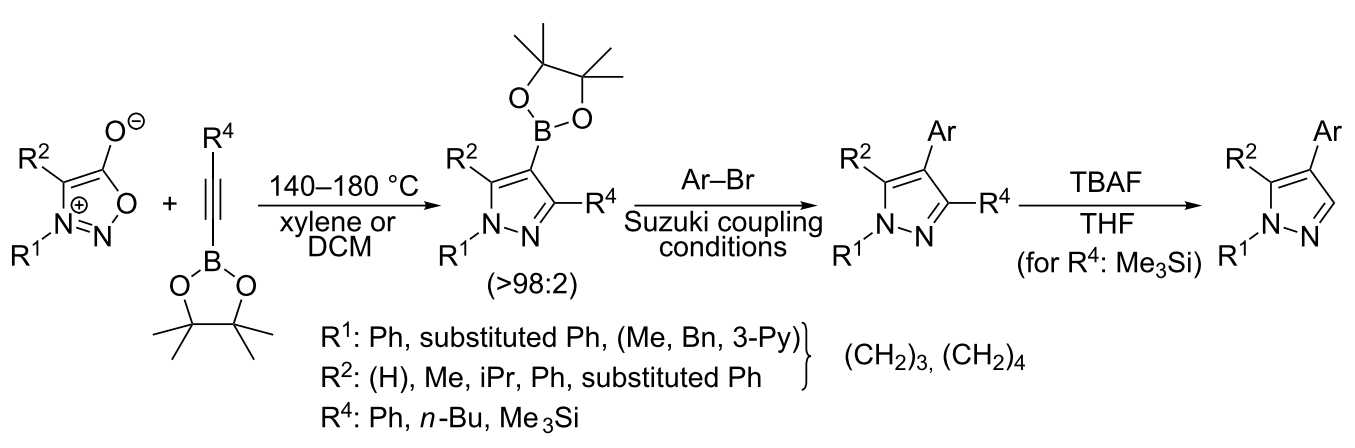

Scheme 10: Synthetic strategy leading to 1,4,5-trisubstituted pyrazoles.

calculations clearly proved the almost apolar character of both possible transition states giving 3- and 4-BPin substituted pyrazoles through bicyclic intermediates (cf. Scheme 5) with a negligible charge transfer flowing from sydnone to the alkyne. This result suggests that there should be a very low influence of the substituents polar effects on the energy of the transition state. Moreover, energy gaps between the dipole HOMO and the dipolarophile LUMO or the dipole LUMO and the dipolarophile HOMO were found to be similar in most cases.

Different reaction course was also observed [112] for 3-alkyl and 3-arylsydnones carrying in position 4 a six-membered heterocyclic ring containing a nitrogen atom adjacent to a linkage with parent sydnone ring (pyridin-2-yl, quinolin-2-yl, 5,6-dihydro-4H-1,3-oxazin-2-yl). Such sydnones reacted with potassium 2-substituted acetylene trifluoroborates under boron trifluoride diethyl etherate catalysis to give corresponding pyrazolo[3',4':4,5][1,2]azaborolo[2,3-a]pyridin-5-ium-4-uides (or quinolin-5-ium-4-uide) in good to excellent yields (Scheme 11). These zwitterionic compounds can be further hydrolyzed to 1,3,5-trisubstituted pyrazoles, oxidized to 4-hydroxy-1,3,5trisubstituted pyrazoles, transformed to 4-BPin derivative of 1,3,5-trisubstituted pyrazole or arylated under palladium catalysis to give 4-aryl-1,3,5-trisubstitutedpyrazole (Scheme 11). Overall therefore, a nitrogen atom in the sydnone 4-heterocyclyl substituent (especially 2-pyridyl) acts as powerful activating group enabling cycloaddition reaction under ambient conditions and also influencing the regioselectivity. Boron carrying two alkynyl groups always appear formally in position 4 of pyrazole ring including reaction with potassium acetylene trifluoroborate.

The last type of non-symmetrical internal alkynes to be considered are cycloalkynes. Their strain-promoted reactions again proceed quickly under mild reaction conditions (cf. section concerning symmetrical internal alkynes) but their regioselectivity is generally low, which is in accordance with the re-

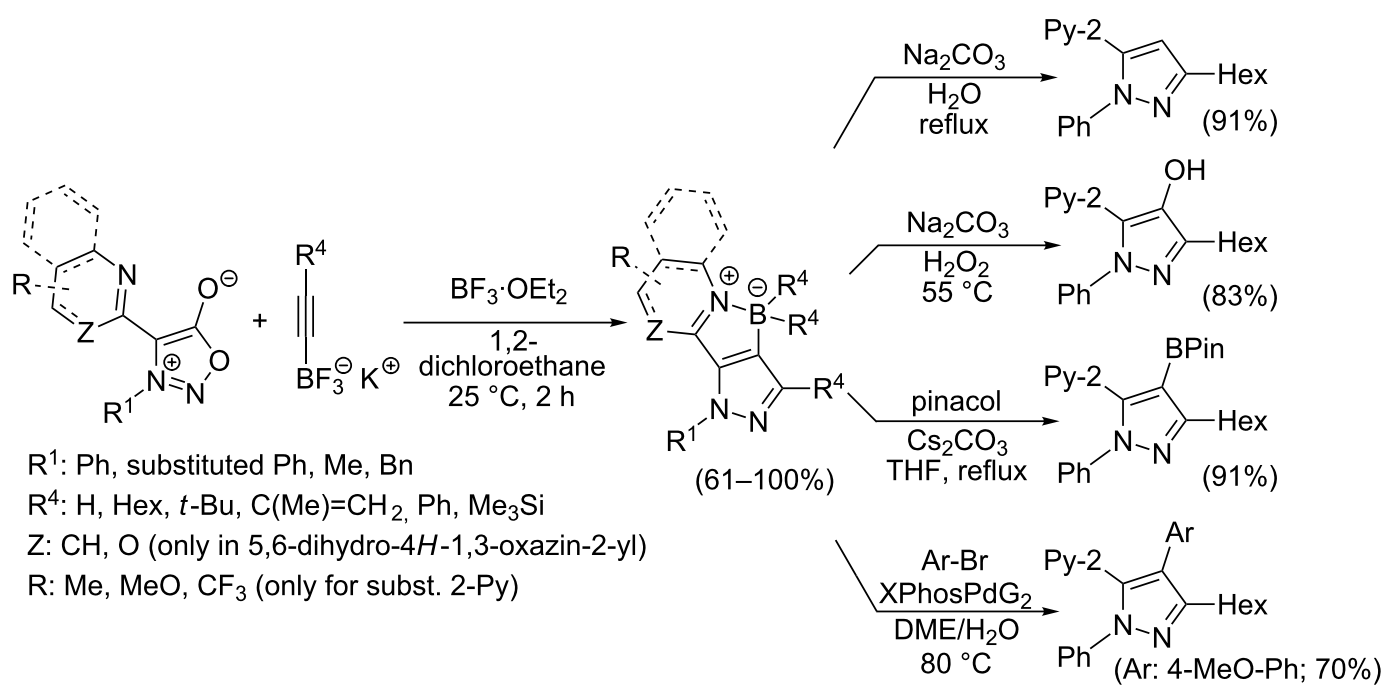


activity-selectivity principle. The first example was described [44] by Taran's group in 2016 when they observed an ultrafast reaction of 6-[11,12-didehydrodibenzo[b,f]azocine-5(6H)-yl]-6oxohexanoic acid with 4-fluoro-3-(4-methylphenyl)sydnone (Scheme 12). Unfortunately the regioselectivity of the reaction was not specified.

An aryne generation (Scheme 13) was also used for the synthesis of a key intermediate of the potent antitumor PARP inhibitor - niraparib - containing an indazole core [113]. A substituted 2,3-aryne was generated in situ from (siloxy)benzocyclobutenes and CsF but the regioselectivity was poor: a ratio of both possible regioisomers of 45:55 was obtained.
A much better regioselectivity was achieved [114] in a reaction of 1,3-/1,4-benzdiyne equivalents (2,4-bissilyl-1,3-bistriflates) with two different dipoles from which one was 3-phenyl-4-(4methoxyphenyl)sydnone (Scheme 14). The reason for the much better regioselectivity probably lies in the steric hindrance between the bulky $t$ - $\mathrm{BuMe}_{2} \mathrm{Si}$ and $4-\mathrm{MeO}-\mathrm{Ph}$ groups.

The in situ generation of arynes or six-membered cycloalkynes from their corresponding trimethylsilyl triflates was recently used by Garg et al. [115] and Bräse et al. [116] in expanding the utility of oxygen- or nitrogen-containing strained heterocycloalkynes (Scheme 15) but the regioselectivity was poor in most cases.

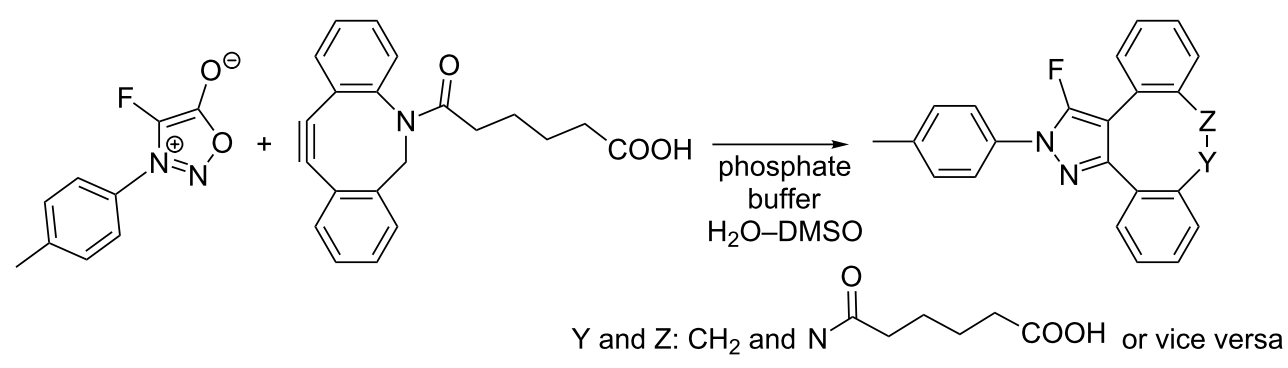

Scheme 12: Strain-promoted sydnone alkyne cycloaddition (SPSAC).<smiles></smiles>

Scheme 13: Synthesis of a key intermediate of niraparib.<smiles></smiles><smiles>COc1ccc(-c2c3c(C)c(OC(C)(C)C)ccc3nn2-c2ccccc2)cc1</smiles>

(85:15) 


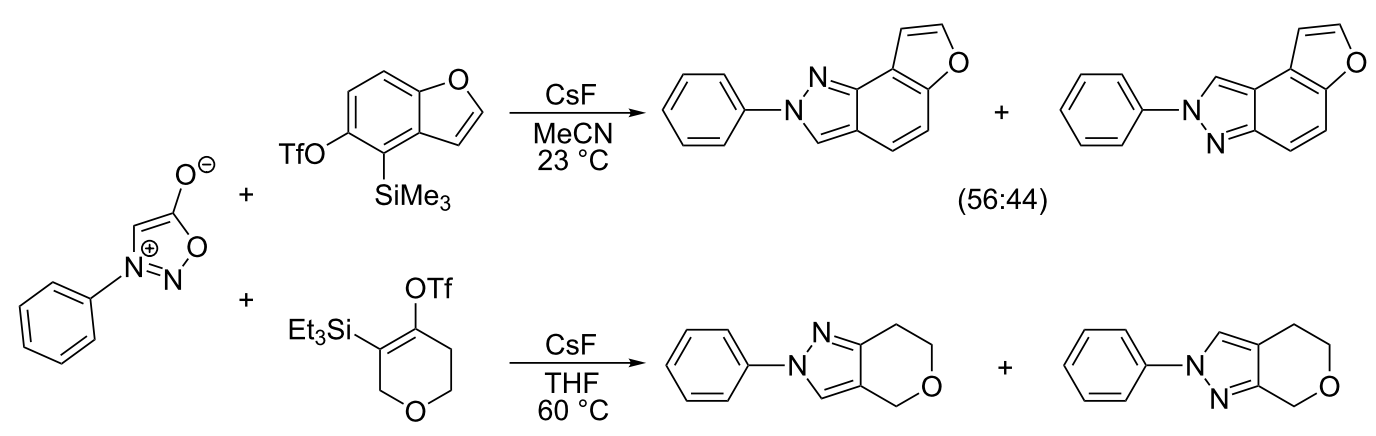

$(40: 60)$<smiles></smiles>

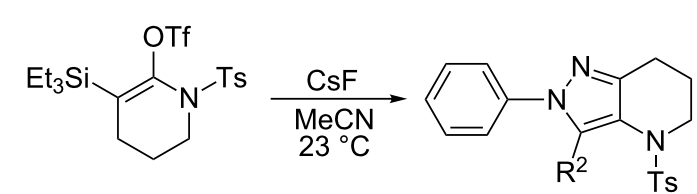<smiles>[R]c1c2c(nn1-c1ccccc1)N([AsH])CCC2</smiles>

$\mathrm{R}^{2}=\mathrm{H}: 84 \%$ (80:20); $\mathrm{R}^{2}=\mathrm{MeCO}: 88 \%$ (29:71); $\mathrm{R}^{2}=\mathrm{CH}_{2}=\mathrm{CH}-\mathrm{CH}_{2}-\mathrm{NHCO}: 94 \%$ (22:78)

$\mathrm{R}^{2}=\mathrm{HC} \equiv \mathrm{CH}-\mathrm{CH}_{2}-\mathrm{NHCO}: 46 \%(22: 78) ; \mathrm{R}^{2}=4-\mathrm{Cl}-\mathrm{PhS}: 68 \%$ (64:36); $\mathrm{R}^{2}=$ quinolin-2-yl: $56 \%$ (50:50)

$\mathrm{R}^{2}=4-\mathrm{Me}-\mathrm{Ph}: 90 \%(56: 44)$

Scheme 15: Reaction of sydnones with heterocyclic strained cycloalkynes.

\section{Photochemical reaction of sydnones with non-symmetrical alkynes}

As mentioned in the previous section, Gotthardt and Reiter $[63,64]$ studied the photochemical reaction of sydnones with terminal alkynes. They have also studied the reaction with phenylacetylene, methyl propiolate and ethyl phenylpropiolate in a batch reactor under irradiation with $300 \mathrm{~nm}$ light (Table 6).
The formation of both regioisomers a and $\mathbf{b}$ was observed when the most reactive methyl propiolate was used as a reactant. Moreover, the ratio (16:84) obtained from starting 3,4-diphenylsydnone is similar with those obtained from 1,3-diphenylnitrilimine independently generated either from 2,5-diphenyltetrazol or from $N$-phenylbenzenecarbohydrazonoyl chloride. This observation clearly supports the mechanism depicted in Scheme 6. The distribution of both regioisomers qualitatively agrees with

Table 6: Photochemical cycloaddition of $N$-phenylsydnones with non-symmetrical alkynes.<smiles></smiles>

$\mathrm{R}^{2}: \mathrm{H}, \mathrm{Me}, \mathrm{Ph}, \mathrm{MeS}, \mathrm{PhS}$

$\mathrm{R}^{3}, \mathrm{R}^{4}$ : H, COOMe; H, Ph; Ph, COOMe<smiles>[R]c1nn(-c2ccccc2)c([R])c1[R]</smiles>

a<smiles>[R]c1nn(-c2ccccc2)c([R])c1[R]</smiles>

b

\begin{tabular}{llllllll}
\hline entry & $\mathrm{R}^{2}$ & $\mathrm{R}^{3}$ & $\mathrm{R}^{4}$ & conditions & ratio $\mathbf{a}: \mathbf{b}$ & yield $\mathbf{a} / \mathbf{b}$ [\%] & ref. \\
\hline 1 & $\mathrm{Ph}$ & $\mathrm{H}$ & $\mathrm{COOMe}$ & benzene, $23 \mathrm{~h}$, light $(300 \mathrm{~nm})$ & $16: 84$ & $12 / 62$ & {$[63,64]$} \\
2 & $\mathrm{Ph}$ & $\mathrm{COOEt}$ & $\mathrm{Ph}$ & $\mathrm{CH}_{2} \mathrm{Cl}_{2}, 27.5 \mathrm{~h}$, light $(300 \mathrm{~nm})$ & $0: 100$ & $0 / 33$ & {$[63,64]$} \\
3 & $\mathrm{Ph}$ & $\mathrm{H}$ & $\mathrm{Ph}$ & $\mathrm{CH}_{2} \mathrm{Cl}_{2}, 66 \mathrm{~h}$, light $(300 \mathrm{~nm})$ & $0: 100$ & $0 / 63$ & {$[63,64]$} \\
4 & $\mathrm{Me}$ & $\mathrm{H}$ & $\mathrm{Ph}$ & $\mathrm{CH}_{2} \mathrm{Cl}_{2}, 62 \mathrm{~h}$, light $(300 \mathrm{~nm})$ & $0: 100$ & $0 / 13$ & {$[64]$} \\
5 & $\mathrm{MeS}$ & $\mathrm{H}$ & $\mathrm{COOMe}$ & $\mathrm{CH}_{2} \mathrm{Cl}_{2}, 27 \mathrm{~h}$, light $(300 \mathrm{~nm})$ & $12: 88$ & $6 / 44$ & {$[64]$} \\
6 & $\mathrm{PhS}$ & $\mathrm{H}$ & COOMe & benzene, $18.5 \mathrm{~h}$, light $(300 \mathrm{~nm})$ & n.d. & $5 / 41$ & {$[64]$}
\end{tabular}


the proposal of Houk et al. [94] combining the dipole HOMO with the dipolarophile LUMO (type-I mechanism).

\section{Copper-catalyzed reaction of sydnones with terminal alkynes}

A substantial breakthrough in the field of 3-arylsydnone-terminal alkyne cycloaddition was achieved by Taran's group in 2013 [3]. They developed a regioselective $\mathrm{Cu}(\mathrm{I})$-phenanthroline-catalyzed variant of this reaction (i.e., copper-catalyzed sydnone alkyne cycloaddition; henceforth called CuSAC) enabling regioselective formation of 1,4-disubstituted pyrazoles under much milder reaction conditions (in various solvents including aqueous solution at $25-60{ }^{\circ} \mathrm{C}$, Table 7) than previously used for its thermal-mediated counterpart. Such mild reaction conditions together with very high and reverse regioselectivity and efficiency (in most cases $85-99 \%$ yields) makes the CuSAC reaction a very good alternative to the well-established azide-alkyne click-reaction [117] useful not only in classical organic synthesis but also in bioconjugation applications. Moreover, a further improvement was later devised by the same authors, which avoids the highly toxic $N$-nitroso- $N$-phenylglycine, (precursor of sydnone) and involves a three-step onepot transformation of starting $N$-phenylglycine to the corresponding pyrazole [118].
There are several limitations of the CuSAC reaction. First, it apparently fails with 3-alkyl sydnones and also with almost all 4-substituted 3-phenylsydnones except 4-F [44], 4-Cl and 4- $\mathrm{Br}$ derivatives [119]. However, this fortunate exception gave the further possibility to exchange halogen (especially bromine) by either an aryl, alkyl or alkenyl group via Suzuki coupling reaction with boronic acids to give otherwise rarely available 1,4,5trisubstituted pyrazoles [119]. The second limitation is that the $\mathrm{CuSAC}$ reaction proceeds only with terminal alkynes. The latter fact clearly indicates some kind of participation of the alkyne's slightly acidic terminal hydrogen in the reaction mechanism. Indeed, as early as in his primary paper [3] Taran suggested formation of $\mathrm{Cu}(\mathrm{I})$ acetylide (for additional information concerning reactions involving $\mathrm{Cu}(\mathrm{I})$ acetylides see references $[121,122])$ as the key species coordinating N2 of the sydnone through the $\mathrm{Cu}$ atom in the transition state. This suggestion was supported by Gomez-Bengoa and Harrity et al. [92] who performed thorough quantum calculation of various transition states involving different modes of interaction between 3-phenylsydnone and $\mathrm{Cu}(\mathrm{I})$ phenylacetylide (Scheme 16) and found Taran's suggestion as the most plausible because of the lowest activation free energy $\left(\Delta G^{\ddagger}=25.4 \mathrm{kcal} \cdot \mathrm{mol}^{-1}\right)$ and due to the observed 1,4-regiocontrol. Intrinsic reaction coordinate (IRC) calculations also showed concerted but asynchronous for-

Table 7: $\mathrm{Cu}(\mathrm{I})$-catalyzed cycloaddition of sydnones with terminal alkynes.

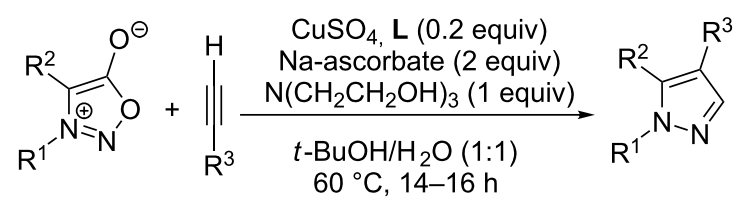

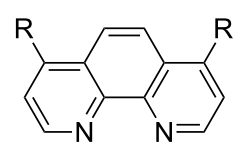

$\mathrm{L}_{1}: \mathrm{R}=4-\mathrm{NaO}_{3} \mathrm{~S}-\mathrm{Ph}$ $\mathrm{L}_{2}: \mathrm{H}$

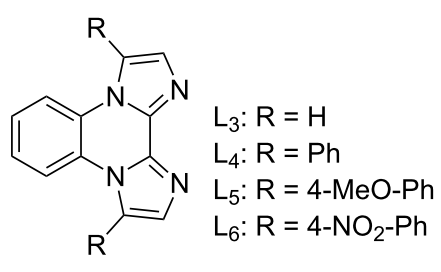

ligand $(L) \quad$ yield [\%] ref.

$\mathrm{L}_{1} \quad 96-98 \quad[3,119,120]$

$\mathrm{L}_{1} \quad 85^{\mathrm{a}} \quad[118]$

$\mathrm{L}_{2} \quad 99 \quad$ [3]

$\mathrm{L}_{1} \quad 80$

[3]

64

$[3,120]$

$[3,120]$

4-MeOPh

2-Py

thiophen-3-yl

1-heptyl

$\mathrm{PhCOOCH}_{2}$

$\left(\mathrm{CH}_{3}\right)_{2} \mathrm{C}(\mathrm{OH})$

$\mathrm{L}_{1}$

$\mathrm{L}_{1}$

95

$69^{a}$

[3]

[118]

$\mathrm{L}_{1} \quad 95$

$L_{1} \quad 61$

$\mathrm{L}_{1} \quad 93$

$[3,120]$

$[3,120]$

$[3,120]$

$[3,120]$

$[3,120]$

COOEt

$\mathrm{PhCH}_{2} \mathrm{CH}_{2}$

$\mathrm{PhCH}_{2} \mathrm{CH}_{2}$

$\left(\mathrm{CH}_{3}\right)_{2} \mathrm{C}(\mathrm{OH})$
[118]

$[3,120]$

[3]

$[3,120]$ 
Table 7: $\mathrm{Cu}(\mathrm{I})$-catalyzed cycloaddition of sydnones with terminal alkynes. (continued)

13 4-COOH-Ph

14

$\mathrm{Ph}$

15

$\mathrm{Ph}$

16

$\mathrm{Ph}$

17

$\mathrm{Ph}$

$\mathrm{H}$

$\mathrm{H}$

$\mathrm{H}$<smiles>CCNS(=O)(=O)c1cccc2c(N(C)C)cccc12</smiles>

$\mathrm{H}$<smiles>O=c1ccc2ccc(I)cc2o1</smiles>

$\mathrm{H}$

$\mathrm{H}$<smiles>CCN(CC)CC</smiles>

$\mathrm{L}_{1} \quad 85$

$\mathrm{L}_{1}$<smiles>CO[C@H]1[C@@H](OC(C)=O)[C@H](OC(C)=O)[C@@H](COC(C)=O)O[C@@H]1OC(C)=O</smiles><smiles>CCOC(=O)[C@H](C)Oc1ccc(Oc2ncc(Cl)cc2F)cc1</smiles>

$\mathrm{L}_{1}$<smiles>[Z17]=Nc1ccc2c(-c3ccc(OS(=O)(=O)NCC)cc3S(=O)(=O)O)c3ccc(=NCC)cc-3oc2c1</smiles>

$\mathrm{H}$<smiles>[3H]c1cn([C@H]2C[C@H](O)[C@@H](CO)O2)c(=O)[nH]c1=O</smiles><smiles>Cc1ccc(C(=O)NCC(=O)N[C@@H](Cc2c[nH]c3ccccc23)C(=O)CCC(=O)O)cc1</smiles>

$\mathrm{H}$<smiles>CCNS(=O)(=O)c1cccc2c(N(C)C)cccc12</smiles>

$\mathrm{L}_{1}$

99

$[3,120]$

$\mathrm{Bn}-\mathrm{N}-\mathrm{Ts}$

$\mathrm{Bn}-\mathrm{N}-\mathrm{Ts}$

$\mathrm{Bn}-\mathrm{N}-\mathrm{Ts}$

$\mathrm{Bn}-\mathrm{N}-\mathrm{Ts}$

$\mathrm{PhCH}_{2} \mathrm{CH}_{2}$

$\mathrm{PhCH}_{2} \mathrm{CH}_{2}$

$\mathrm{PhCH}_{2} \mathrm{CH}_{2}$

$\mathrm{PhCH}_{2} \mathrm{CH}_{2}$

$\mathrm{PhCH}_{2} \mathrm{CH}_{2}$

$\mathrm{PhCH}_{2} \mathrm{CH}_{2}$

$\mathrm{PhCH}_{2} \mathrm{CH}_{2}$

$\mathrm{PhCH}_{2} \mathrm{CH}_{2}$

$\mathrm{PhCH}_{2} \mathrm{CH}_{2}$

$\mathrm{PhCH}_{2} \mathrm{CH}_{2}$

$\mathrm{Ph}$

99

$[3,120]$

[3]

$[3,120]$

96

$[3,120]$

62
$55^{a}$

$[3,120]$

[118]

92

$[3,120]$

84

$[3,120]$

4-CF $-\mathrm{Ph}$

naphthalen-1-yl

2-COOMe-thiophen-3-yl

$\mathrm{Ph}$

$\mathrm{H}$

$\mathrm{L}_{2}$

[116]

[116]

[116]

[116]

[118]

[118]

[118]

[118]

[118]

[118]

[118]

[118]

[118]

[118]

[118] 


\begin{tabular}{|c|c|c|c|c|c|c|}
\hline 36 & $\mathrm{Ph}$ & $\mathrm{H}$ & $n$-pentyl & $\mathrm{L}_{1}$ & $82^{a}$ & [118] \\
\hline 37 & $\mathrm{Ph}$ & $\mathrm{H}$ & $\mathrm{CH}_{2} \mathrm{NHCOO}-t-\mathrm{Bu}$ & $\mathrm{L}_{1}$ & $85^{a}$ & [118] \\
\hline 38 & $\mathrm{Ph}$ & $\mathrm{H}$ & & $\mathrm{L}_{1}$ & $91^{a}$ & [118] \\
\hline 39 & $\mathrm{Ph}$ & $\mathrm{Br}$ & $\mathrm{PhCH}_{2} \mathrm{CH}_{2}$ & $\begin{array}{l}\mathrm{L}_{1} \\
\mathrm{~L}_{2} \\
\mathrm{~L}_{3} \\
\mathrm{~L}_{4} \\
\mathrm{~L}_{5} \\
\mathrm{~L}_{6}\end{array}$ & $\begin{array}{l}74^{b} \\
67^{c} \\
60^{d} \\
75 \\
74 \\
13\end{array}$ & $\begin{array}{l}{[119,120]} \\
{[119]} \\
{[119]} \\
{[119]} \\
{[119]} \\
{[119]}\end{array}$ \\
\hline 40 & 4-Me-Ph & $\mathrm{Br}$ & $\mathrm{PhCH}_{2} \mathrm{CH}_{2}$ & $\mathrm{~L}_{4}$ & 80 & [119] \\
\hline 41 & 4-MeO-Ph & $\mathrm{Br}$ & $\mathrm{PhCH}_{2} \mathrm{CH}_{2}$ & $\mathrm{~L}_{4}$ & 70 & {$[119,120]$} \\
\hline 42 & $4-\mathrm{F}-\mathrm{Ph}$ & $\mathrm{Br}$ & $\mathrm{PhCH}_{2} \mathrm{CH}_{2}$ & $\mathrm{~L}_{4}$ & 55 & [119] \\
\hline 43 & 4-I-Ph & $\mathrm{Br}$ & $\mathrm{PhCH}_{2} \mathrm{CH}_{2}$ & $\mathrm{~L}_{4}$ & 72 & [119] \\
\hline 44 & $\mathrm{Ph}$ & $\mathrm{Br}$ & COOEt & $\mathrm{L}_{4}$ & 38 & [119] \\
\hline 45 & $\mathrm{Ph}$ & $\mathrm{Br}$ & $\mathrm{Ph}$ & $\mathrm{L}_{4}$ & 63 & [119] \\
\hline 46 & $\mathrm{Ph}$ & $\mathrm{Br}$ & 6-MeO-naphthalen-2-yl & $\mathrm{L}_{4}$ & 77 & [119] \\
\hline 47 & $\mathrm{Ph}$ & $\mathrm{Br}$ & 4-MeO-Ph & $\mathrm{L}_{4}$ & 44 & [119] \\
\hline 48 & $\mathrm{Ph}$ & $\mathrm{Br}$ & $\mathrm{CH}_{2} \mathrm{NHCOO}-\mathrm{t}-\mathrm{Bu}$ & $\mathrm{L}_{4}$ & 69 & [119] \\
\hline 49 & $\mathrm{Ph}$ & $\mathrm{Br}$ & $\mathrm{CH}_{2} \mathrm{OCOPh}$ & $\mathrm{L}_{4}$ & 52 & [119] \\
\hline 50 & $\mathrm{Ph}$ & $\mathrm{Br}$ & $\mathrm{BrCH}_{2} \mathrm{CH}_{2}$ & $\begin{array}{l}\mathrm{L}_{1} \\
\mathrm{~L}_{4}\end{array}$ & $\begin{array}{l}45^{a} \\
63\end{array}$ & [119] \\
\hline 51 & quinolin-5-yl & $\mathrm{Br}$ & $\mathrm{PhCH}_{2} \mathrm{CH}_{2}$ & $\mathrm{~L}_{4}$ & 33 & [119] \\
\hline 52 & $\mathrm{Ph}$ & $\mathrm{Br}$ & & $\mathrm{L}_{4}$ & 52 & [119] \\
\hline 53 & $\mathrm{Ph}$ & $\mathrm{Br}$ & & $\mathrm{L}_{4}$ & 65 & [119] \\
\hline 54 & $\mathrm{Ph}$ & $\mathrm{Me}$ & $\mathrm{PhCH}_{2} \mathrm{CH}_{2}$ & $\mathrm{~L}_{1}$ & 7 & [119] \\
\hline 55 & $\mathrm{Ph}$ & $\mathrm{Cl}$ & $\mathrm{PhCH}_{2} \mathrm{CH}_{2}$ & $\mathrm{~L}_{1}$ & $80^{e}$ & [119] \\
\hline 56 & $\mathrm{Ph}$ & $\mathrm{CN}$ & $\mathrm{PhCH}_{2} \mathrm{CH}_{2}$ & $\mathrm{~L}_{1}$ & $10^{f}$ & [119] \\
\hline
\end{tabular}

${ }^{a}$ One-pot protocol starting from corresponding $N$-phenyl glycine; ${ }^{b}$ ratio $1,4,5: 1,3,5$ is $83: 17$; ${ }^{c}$ ratio $1,4,5: 1,3,5: 1,4,5$-debrominated product is $83: 10: 7$; dratio $1,4,5: 1,3,5: 1,4,5$-debrominated product is $97: 0: 3$; eratio $1,4,5: 1,3,5$ is $96: 4$; ${ }^{f}$ ratio $1,4,5: 1,3,5$ is $50: 50$.

mation of the pyrazole ring, through initial $\mathrm{C}-\mathrm{C}$ bond formation followed by $\mathrm{Cu}-\mathrm{N}$ dissociation and $\mathrm{C}-\mathrm{N}$ bond formation. Experiments performed in $t$-BuOD/ $\mathrm{D}_{2} \mathrm{O}$ [119] also showed almost exclusive (>98:2) deuteration of position 3 in the final pyrazole ring. This finding supports the idea of $\mathrm{Cu}(\mathrm{I})$-acetylide addition to give 3-metalated pyrazole (Cu-pyrazolide) that is, in deuteric solvent hydrolyzed to give 3-deutero pyrazole.

However, Fokin et al. has recently [123] revealed that monomeric copper acetylide complexes are not reactive toward organic azides in analogous copper-catalyzed alkyne-azide cycloaddition $(\mathrm{CuAAC})$ and the catalysis by an external $\mathrm{Cu}(\mathrm{I})$ salt is necessary. This means that a dinuclear copper complex most probably copper(I) acetylide bearing the $\pi$-bound copper salt - plays a key role during the cycloaddition step. On the basis of a crossover experiment with an isotopically enriched ${ }^{63} \mathrm{Cu}(\mathrm{I})$ salt it was concluded that the $\mathrm{CuAAC}$ involves addition of azide nitrogen N3 to $\pi$-bound copper of dinuclear copper complex with concerted addition of alkyne $\beta$-carbon to azide terminal nitrogen N1. An intermediate formed in which N3 is coordinated to both copper atoms undergoes fast ligand exchange between both copper atoms which makes them equivalent. Then the same N3 coordinating both $\mathrm{Cu}$ atoms attacks the terminal carbon of the polarized double bond with concerted 


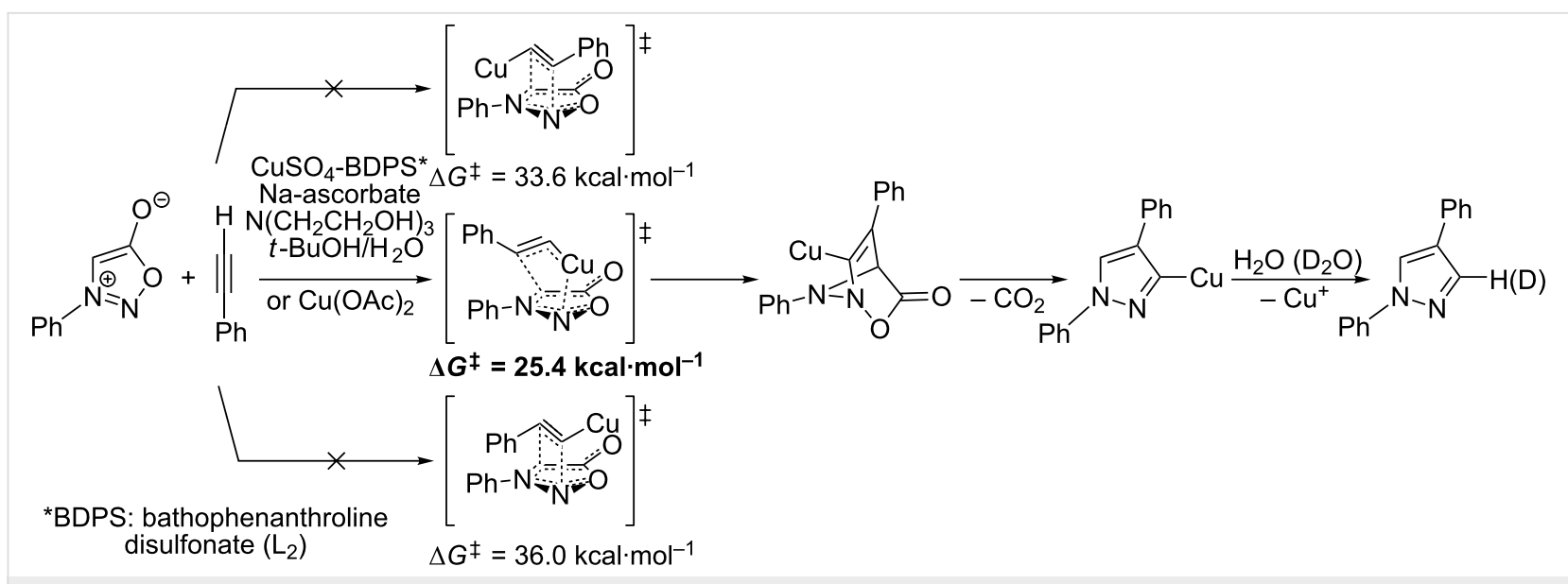

Scheme 16: Mono-copper catalyzed cycloaddition reaction.

cleavage of one of the two copper atoms. The copper triazolide formed in this way is then hydrolyzed to the final triazole. The same presumption (Scheme 17) concerning the role of the two $\mathrm{Cu}$ atoms was also adopted by Taran in his newer paper [119] but no experimental evidence for this mechanism has been given yet.

From previous studies it is known that for the spherically symmetric $\mathrm{d}^{10} \mathrm{Cu}(\mathrm{I})$ ion, the common geometries are two-coordinate linear, three-coordinate trigonal planar, and four-coordinate tetrahedral [124]. Phenanthrolines form with $\mathrm{Cu}(\mathrm{I})$ at $1: 1$ ratio three-coordinated trigonal planar complexes or at 2:1 ratio tetra-coordinated tetrahedral complexes [125]. If $\mathrm{Cu}_{2}(\mathrm{CN})_{2}$ (in which $\mathrm{CN}$ is isoelectronic with acetylide) is employed as a $\mathrm{Cu}(\mathrm{I})$ source then three-coordinated trigonal planar polymeric arrangement was observed [126]. From this observations it appears that mono- or dimeric three-coordinated trigonal planar $\mathrm{Cu}(\mathrm{I})$-acetylide-phenanthroline complex should be the reactive species during CuSAC. This idea was supported by the fact that during the reaction of 4-bromosydnones with 4-phenylbut-1-yne [119] tridentate tris(benzimidazole) ligands completely failed and tris(triazole) ligands gave only poor to moderate yields $(16-65 \%)$ even at $100{ }^{\circ} \mathrm{C}$, whereas all the bidentate ligands (phenanthrolines $\mathrm{L}_{1}, \mathrm{~L}_{2}$ and diimidazo[1,2$\left.a: 2^{\prime}, 1^{\prime}-c\right]$ quinoxalines $\mathrm{L}_{3}-\mathrm{L}_{6}$ ) were found to be more efficient both in terms of the isolated yield as well as the regioselectivity (see entry 39 in Table 7). From the comparison of phenanthro- line $\left(\mathrm{L}_{1}, \mathrm{~L}_{2}\right)$ and diimidazo[1,2- $\left.a: 2^{\prime}, 1^{\prime}-c\right]$ quinoxaline $\left(\mathrm{L}_{3}-\mathrm{L}_{6}\right)$ complexes it appears that the higher angle between the two coordinative nitrogen atoms may have a positive impact on the catalytic efficiency.

Gomez-Bengoa and Harrity et al. [92] also inspected the role of $\mathrm{Cu}(\mathrm{I}) / \mathrm{Cu}$ (II) salts as well as other Lewis acids which could strengthen the electrophilicity of the starting sydnone under thermal reaction conditions. They found two competitive catalytic routes leading to different cycloaddition products. According to their original presumption some Lewis acids (TMSOTf $<$ $\left.\mathrm{Zn}(\mathrm{OAc})_{2}<\mathrm{MgBr}_{2}<\mathrm{Cu}(\mathrm{OTf})_{2}\right)$ catalyzed the thermal reaction of phenylsydnone with phenylacetylene to give the expected 1,3-diphenylpyrazole in a ratio $>10: 1$ over the 1,4-diphenyl isomer. Quantum calculations and IR measurements performed for the most active $\mathrm{Cu}(\mathrm{OTf})_{2}$ have shown that this salt coordinates to the sydnone oxygen carrying a negative charge which leads to an energy decrease of the sydnone LUMO and an increase of its electrophilicity. Also computed activation free energy $\left(\Delta G^{\ddagger}=25.4 \mathrm{kcal} \cdot \mathrm{mol}^{-1}\right)$ for the rate-limitting $[3+2]$-cycloaddition step leading to the 1,3 -isomer was substantially lower if compared to the uncatalyzed reaction pathway $\left(\Delta G^{\star}=32.5 \mathrm{kcal} \cdot \mathrm{mol}^{-1}\right)$.

If other $\mathrm{Cu}$ (II) salts were used as a catalyst then the ratio of 1,3-/1,4-isomers gradually changed from 90:10 to 3:97 (Table 8).

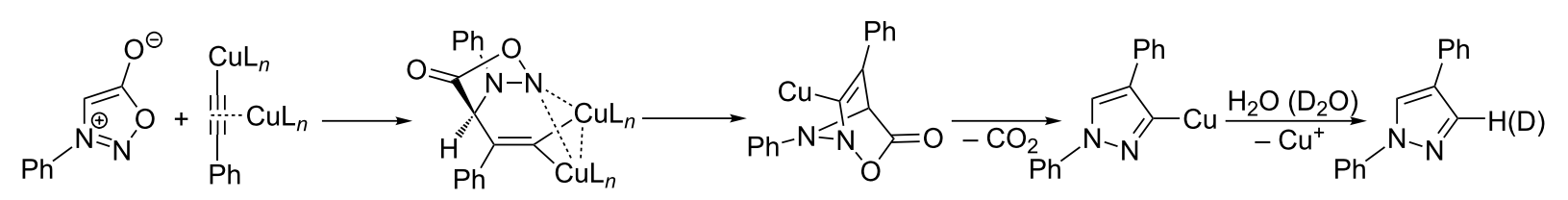




\begin{tabular}{|c|c|c|}
\hline copper salt ${ }^{\mathrm{a}}$ & conversion after $20 \mathrm{~min}$. in o-DCB at $140^{\circ} \mathrm{C}(\%)$ & ratio 1,3-/1,4-pyrazole \\
\hline $\mathrm{Cu}(\mathrm{OTf})_{2}$ & 100 & $90: 10$ \\
\hline $\mathrm{Cu}(\mathrm{TFA})_{2}$ & 75 & $80: 20$ \\
\hline $\mathrm{Cu}\left(\mathrm{BF}_{4}\right)_{2}$ & 44 & $64: 36$ \\
\hline $\mathrm{CuCl}_{2} \cdot 4 \mathrm{H}_{2} \mathrm{O}$ & 74 & $48: 52$ \\
\hline $\mathrm{CuCO}_{3}$ & 29 & $27: 73$ \\
\hline $\mathrm{Cu}(\mathrm{acac})_{2}$ & 19 & $20: 80$ \\
\hline $\mathrm{CuBr}_{2}$ & 91 & 19:81 \\
\hline $\mathrm{Cu}(\mathrm{OAc})_{2}$ & 39 & $17: 83$ \\
\hline $\mathrm{Cu}(2-\text { Et-hexanoate })_{2}$ & 88 & $8: 92$ \\
\hline $\mathrm{Cu}(\mathrm{hfacac})_{2}$ & 13 & $3: 97$ \\
\hline
\end{tabular}

aacac - acetylacetonate; hfacac - hexafluoroacetylacetonate; TFA - trifluoroacetate.

A completely different ratio of both isomers was observed when $\mathrm{Cu}(\mathrm{II})$ carboxylates and acetylacetonates were employed instead of $\mathrm{Cu}(\mathrm{OTf})_{2}$. This was explained by different operating mechanisms. While $\mathrm{Cu}(\mathrm{OTf})_{2}, \mathrm{Cu}(\mathrm{TFA})_{2}$ and $\mathrm{Cu}\left(\mathrm{BF}_{4}\right)_{2}$ behave mainly as Lewis acids, other $\mathrm{Cu}(\mathrm{II})$ salts/complexes preferentially oxidize one equivalent of phenylacetylene to give 1,4diphenylbuta-1,3-diyne (isolated in $80 \%$ yield) and the evolved $\mathrm{Cu}(\mathrm{I})$ salt then forms $\mathrm{Cu}(\mathrm{I})$ acetylide with a second equivalent of phenylacetylene. Thus formed $\mathrm{Cu}(\mathrm{I})$ acetylide is then responsible for gradual increasing of 1,4-pyrazole occurrence. Quantum calculations [92] and IR measurements performed for $\mathrm{Cu}(\mathrm{OAc})_{2}$ also show that the Lewis acid character of this salt is less pronounced and formation of the 1,3-diphenylpyrazole necessitates a much higher activation free energy $\left(\Delta G^{*}=41.4 \mathrm{kcal} \cdot \mathrm{mol}^{-1}\right)$ than for the uncatalyzed reaction. Formation of 1,4-diphenylpyrazole through $\mathrm{Cu}(\mathrm{I})$-acetylide addition is then the clearly preferred reaction pathway. Moreover, $\mathrm{Cu}(\mathrm{OAc})_{2}$ acts as a very good catalyst not only in the reaction of parent phenylsydnone with phenylacetylene [92]. After appropriate prolongation of the reaction time it delivers the corresponding 1,4-disubstituted pyrazoles in good to excellent yields and with a regioselectivity ratio exceeding 95:5 (Table 9). It is worth noting that 3-benzyl sydnone (representative of otherwise unreactive 3-alkylsydnones) reacts with the highly reactive ethyl propiolate to give ethyl 1-benzylpyrazole4-carboxylate in good yield.

\begin{tabular}{|c|c|c|c|c|c|}
\hline & & $\begin{array}{l}\|_{\mathrm{R}^{3}}^{\mathrm{H}} \\
2 \text { equiv }\end{array}$ & $\frac{\left.\mathrm{Cu}(\mathrm{OAc})_{2}\right) \cdot \mathrm{H}_{2} \mathrm{O}}{\mathrm{o}-\mathrm{DCB}, 140{ }^{\circ} \mathrm{C}}$ & 的 & \\
\hline entry & $\mathrm{R}^{1}$ & $\mathrm{R}^{3}$ & reaction time $(\mathrm{h})$ & ratio $1,3: 1,4$ & yield $[\%]$ \\
\hline 1 & 4-MeO-Ph & $\mathrm{Ph}$ & 5 & $<5: 95$ & 53 \\
\hline 2 & $\mathrm{Ph}$ & COOEt & 1 & $<5: 95$ & 93 \\
\hline 3 & 4-MeO-Ph & COOEt & 2 & $<5: 95$ & 81 \\
\hline 4 & $\mathrm{Ph}$ & $n$-Hex & 3.5 & $<5: 95$ & 73 \\
\hline 5 & 4-MeO-Ph & $n$-Hex & 3.5 & $<5: 95$ & 54 \\
\hline 6 & $\mathrm{Ph}$ & cyclo-Hex & 4 & $<5: 95$ & 100 \\
\hline 7 & 4-MeO-Ph & cyclo-Hex & 2.5 & $<5: 95$ & 71 \\
\hline 8 & $\mathrm{Ph}$ & cyclohex-1-enyl & 2.5 & $<5: 95$ & 71 \\
\hline 9 & 4-MeO-Ph & cyclohex-1-enyl & 2.5 & $<5: 95$ & 60 \\
\hline 10 & $\mathrm{Ph}$ & cyclopropyl & 4 & $<5: 95$ & 96 \\
\hline 11 & $\mathrm{Ph}$ & thiophen-3-yl & 2.5 & $<5: 95$ & 95 \\
\hline 12 & 4-F-Ph & COOEt & 4 & $<5: 95$ & 95 \\
\hline 13 & $\mathrm{Bn}$ & COOEt & 4 & $<5: 95$ & 60 \\
\hline
\end{tabular}


Copper(II) acetate anchored on a modified silica gel can also serve as an efficient catalyst in batch reactor or if housed in stainless steel cartridges [127] in continuous-flow conditions (Table 10). Again, the 4-substituted pyrazole is preferentially formed.

\section{Conclusion}

Since its discovery in the sixties of the last century, the thermal $[3+2]$-cycloaddition of sydnones with alkynes represents a valuable synthetic tool for the preparation of polysubstituted pyrazoles and indazoles despite the limitations: the need for high temperatures $\left(90-170{ }^{\circ} \mathrm{C}\right.$ ) and sometimes poorer regioselectivity. These obstacles can be surpassed either by suitable substitution (activating electron-withdrawing groups, removable silyl or carboxylate groups or replaceable boronic esters) or by efficient catalysis using Lewis acids. Preferential formation of 1,3-di- or 1,3,5-trisubstituted pyrazoles ( $>90: 10)$ is observed in most cases when a terminal alkyne was used as a reactant. On the other hand, the recent discovery of $\mathrm{Cu}(\mathrm{I})$ catalysis in the sydnone-alkyne cycloaddition (CuSAC) enables regioselective formation of complementary 1,4-disubstituted or 5-halogeno1,4-disubstituted pyrazoles under very mild reaction conditions (aqueous $t$ - $\mathrm{BuOH}$ solution at $60{ }^{\circ} \mathrm{C}$ ) and can be considered as a good illustration of the click-reaction. Another important example of sydnone cycloaddition involves a very fast reaction with strained seven- or eight-membered cycloalkynes (strainpromoted sydnone alkyne cycloaddition; SPSAC) which takes place without any catalyst and at ambient temperature. Such mild reaction conditions, (ultra) fast and unambiguous product formation make SPSAC useful in bio-orthogonal applications and competitive in comparison with analogous strain-promoted azide-alkyne cycloaddition (SPAAC). The last possibility of how to influence the cycloaddition between sydnones and alkynes involves photochemical performance of this reaction. Under UV-irradiation sydnones form the corresponding unstable nitrilimines which then undergo $[3+2]$-cycloaddition to give pyrazoles carrying substituents originating from alkynes in positions 4 and 5 instead of 3 and 4 . Yields of this photochemical reaction are mostly lower than $50 \%$ which makes this method less convenient.

Table 10: The solid-supported CuSAC reaction in batch or flow reactor.

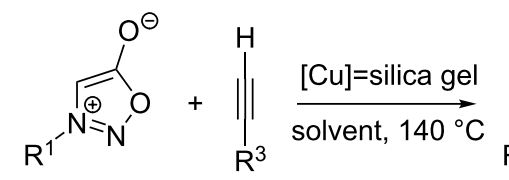

$\mathrm{R}^{1^{-N}-N^{3}}$

[Cu]=silica gel:

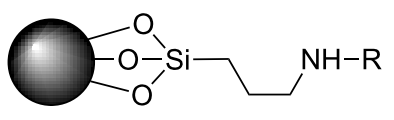

$\mathrm{R}: \mathrm{H}, \mathrm{CH}_{2} \mathrm{CH}_{2} \mathrm{NH}_{2}$

\begin{tabular}{|c|c|c|c|c|c|}
\hline entry & $\mathrm{R}^{1}$ & $\mathrm{R}^{3}$ & solvent & reaction (residence) time & yield [\%] \\
\hline 1 & $\mathrm{Ph}$ & $\mathrm{Ph}$ & $\begin{array}{l}o-D C B \\
\text { toluene }\end{array}$ & $\begin{array}{l}2 \mathrm{~h} \\
(5 \mathrm{~min})\end{array}$ & $\begin{array}{l}100 \\
100\end{array}$ \\
\hline 2 & $\mathrm{Ph}$ & COOEt & $\begin{array}{l}\text { o-DCB } \\
\text { toluene }\end{array}$ & $\begin{array}{l}6 \mathrm{~h} \\
(5 \mathrm{~min})\end{array}$ & $\begin{array}{l}71 \\
95\end{array}$ \\
\hline 3 & $\mathrm{Ph}$ & cyclopentyl & $\begin{array}{l}o-D C B \\
\text { toluene }\end{array}$ & $\begin{array}{l}6 \mathrm{~h} \\
(15 \mathrm{~min})\end{array}$ & $\begin{array}{l}33 \\
73\end{array}$ \\
\hline 4 & $\mathrm{Ph}$ & $\mathrm{CH}_{2} \mathrm{OH}$ & $\begin{array}{l}\text { o-DCB } \\
\text { toluene }\end{array}$ & $\begin{array}{l}20 \mathrm{~h} \\
(15 \mathrm{~min})\end{array}$ & $\begin{array}{l}69 \\
18\end{array}$ \\
\hline 5 & $\mathrm{Ph}$ & $2-P y$ & $\begin{array}{l}\text { o-DCB } \\
\text { toluene }\end{array}$ & $\begin{array}{l}7 \mathrm{~h} \\
(5 \mathrm{~min})\end{array}$ & $\begin{array}{l}70 \\
24\end{array}$ \\
\hline 6 & 4-MeO-Ph & $\mathrm{Ph}$ & $\begin{array}{l}o-D C B \\
\text { toluene }\end{array}$ & $\begin{array}{l}5 \mathrm{~h} \\
(5 \mathrm{~min})\end{array}$ & $\begin{array}{l}85 \\
75\end{array}$ \\
\hline 7 & 4-MeO-Ph & COOEt & $\begin{array}{l}o-D C B \\
\text { toluene }\end{array}$ & $\begin{array}{l}6 \mathrm{~h} \\
(5 \mathrm{~min})\end{array}$ & $\begin{array}{l}47 \\
56\end{array}$ \\
\hline 8 & 4-MeO-Ph & cyclopentyl & $\begin{array}{l}\text { o-DCB } \\
\text { toluene }\end{array}$ & $\begin{array}{l}6 \mathrm{~h} \\
(15 \mathrm{~min})\end{array}$ & $\begin{array}{l}28 \\
24\end{array}$ \\
\hline 9 & 4-MeO-Ph & $\mathrm{CH}_{2} \mathrm{OH}$ & $\begin{array}{l}o \text {-DCB } \\
\text { toluene }\end{array}$ & $\begin{array}{l}20 \mathrm{~h} \\
(15 \mathrm{~min})\end{array}$ & $\begin{array}{l}55 \\
33\end{array}$ \\
\hline 10 & 4-MeO-Ph & $2-P y$ & $\begin{array}{l}o-D C B \\
\text { toluene }\end{array}$ & $\begin{array}{l}7.5 \mathrm{~h} \\
(10 \mathrm{~min})\end{array}$ & $\begin{array}{l}68 \\
26\end{array}$ \\
\hline 11 & $\mathrm{Bn}$ & $\mathrm{Ph}$ & $\begin{array}{l}\text { o-DCB } \\
\text { toluene }\end{array}$ & $\begin{array}{l}16 \mathrm{~h} \\
(5 \mathrm{~min})\end{array}$ & $\begin{array}{l}47 \\
77\end{array}$ \\
\hline 12 & $\mathrm{Bn}$ & COOEt & o-DCB & $16 \mathrm{~h}$ & 21 \\
\hline
\end{tabular}




\section{ORCID ${ }^{\circledR}$ iDs}

Jiři Váňa - https://orcid.org/0000-0003-4756-7314

Jiří Hanusek - https://orcid.org/0000-0003-2202-1251

\section{References}

1. Huisgen, R.; Gotthardt, H.; Grashey, R. Angew. Chem., Int. Ed. Engl. 1962, 1, 49. doi:10.1002/anie.196200491

2. Huisgen, R.; Gotthardt, H.; Grashey, R. Chem. Ber. 1968, 101, 536-551. doi:10.1002/cber.19681010223

3. Kolodych, S.; Rasolofonjatovo, E.; Chaumontet, M.; Nevers, M.-C.; Créminon, C.; Taran, F. Angew. Chem., Int. Ed. 2013, 52, 12056-12060. doi:10.1002/anie.201305645

4. Decuypère, E.; Plougastel, L.; Audisio, D.; Taran, F. Chem. Commun. 2017, 53, 11515-11527. doi:10.1039/C7CC06405E

5. Dickopp, H. Chem. Ber. 1974, 107, 3036-3042. doi:10.1002/cber.19741070926

6. Badami, B. V.; Puranik, G. S. Indian J. Chem. 1974, 12, 671-673.

7. Badami, B. V.; Puranik, G. S. Can. J. Chem. 1975, 53, 913-914. doi:10.1139/v75-127

8. Gotthardt, H.; Reiter, F. Chem. Ber. 1979, 112, 1193-1205. doi:10.1002/cber.19791120414

9. Gotthardt, H.; Reiter, F. Chem. Ber. 1981, 114, 2450-2464. doi:10.1002/cber.19811140712

10. Maffrand, J.-P. Heterocycles 1981, 16, 35-37. doi:10.3987/R-1981-01-0035

11. Dambal, D. B.; Badami, B. V.; Puranik, G. S. Indian J. Chem., Sect. B 1982, 21, 865-868.

12. Tikare, R. K.; Badami, B. V.; Puranik, G. S. Indian J. Chem., Sect. B 1983, 22, 673-677.

13. Ranganathan, D.; Bamezai, S. Tetrahedron Lett. 1983, 24 , 1067-1070. doi:10.1016/S0040-4039(00)81606-8

14. Havanur, S. B.; Puranik, G. S. Indian J. Chem., Sect. B 1985, 24 , 864-866.

15. Collibee, W. L.; Anselme, J.-P. Bull. Soc. Chim. Belg. 1986, 95, 655-662. doi:10.1002/bscb.19860950809

16. Kano, K.; Scarpetti, D.; Warner, J. C.; Anselme, J.-P.; Springer, J. P.; Arison, B. H. Can. J. Chem. 1986, 64, 2211-2219. doi:10.1139/v86-364

17. Badachikar, S. V.; Tikare, R. K.; Puranik, G. S. Indian J. Chem., Sect. B 1986, 25, 1079-1080.

18. Kalinin, V. N.; Min, S. F. Bull. Acad. Sci. USSR, Div. Chem. Sci. (Engl. Transl.) 1988, 37, 762-764. doi:10.1007/BF01455497

19. Sakamoto, T.; Shiga, F.; Uchiyama, D.; Kondo, Y. Heterocycles 1992, 33, 813-818. doi:10.3987/COM-91-S87

20. Dumitraşcu, F.; Drăghici, C.; Dumitrescu, D.; Tarko, L.; Răileanu, D. Liebigs Ann./Recl. 1997, 2613-2616. doi:10.1002/jlac.199719971229

21. Dumitraşcu, F.; Drăghici, C.; Căproiu, M.; Crangus, C.; Mitan, C. I.; Barbu, L.; Răileanu, D. Rev. Chim. (Bucharest, Rom.) 2001, 52, 183-187.

22. Dumitraşcu, F.; Mitan, C. I.; Dumitrescu, D.; Drăghici, C.; Căproiu, M. T. ARKIVOC 2002, No. ii, 80-86. doi:10.3998/ark.5550190.0003.209

23. Dumitraşcu, F.; Mitan, C. I.; Drăghici, C.; Barbu, L.; Căproiu, M. T. A. Univ. Bucuresti, Chim. 2002, 11, 237-242.

24. Dumitraşcu, F.; Drăghici, C.; Crangus, C.; Căproiu, M.; Mitan, C. I.; Dumitrescu, D.; Răileanu, D. Rev. Roum. Chim. 2002, 47, 315-318.
25. Dumitraşcu, F.; Mitan, C. I.; Dumitrescu, D.; Barbu, L.; Hrubaru, M.; Caprau, D.; Vuluga, D. Rev. Chim. (Bucharest, Rom.) 2003, 54, 747-751.

26. Chang, E.-M.; Lin, C.-J.; Wong, F. F.; Yeh, M.-Y. Heterocycles 2006, 68, 733-748. doi:10.3987/COM-06-10699

27. Sanyal, R.; Badami, B. V. J. Heterocycl. Chem. 2006, 43, 827-834. doi:10.1002/jhet.5570430403

28. Dumitraşcu, F.; Drăghici, C.; Vuluga, D.; Căproiu, M. T. Rev. Roum. Chim. 2006, 51, 255-260.

29. Chang, E.-M.; Lee, C.-T.; Chen, C.-Y.; Wong, F. F.; Yeh, M.-Y. Aust. J. Chem. 2008, 61, 342-349. doi:10.1071/CH07381

30. Rodriguez, A.; Moran, W. J. Synthesis 2009, 650-654. doi:10.1055/s-0028-1083344

31. Chang, E.-M.; Huang, S.-L.; Lee, C.-T.; Lin, H.-C.; Chen, C.-Y.; Huang, Y.-Y.; Lin, S.-K.; Wong, F. F. Aust. J. Chem. 2009, 62, 1355-1362. doi:10.1071/CH08344

32. Foster, R. S.; Jakobi, H.; Harrity, J. P. A. Org. Lett. 2012, 14, 4858-4861. doi:10.1021/ol3021918

33. Foster, R. S.; Adams, H.; Jakobi, H.; Harrity, J. P. A. J. Org. Chem. 2013, 78, 4049-4064. doi:10.1021/jo400381a

34. Nassoy, A.-C.; Raubo, P.; Harrity, J. P. A. Tetrahedron Lett. 2013, 54, 3094-3096. doi:10.1016/j.tetlet.2013.03.139

35. Wang, L.-Y.; Chang, E.-C.; Yeh, M.-Y.; Chung, Y. H.; Huang, J.-J.; Wong, F. F. Heteroat. Chem. 2014, 25, 171-177. doi:10.1002/hc. 21151

36. Lopes, S. M. M.; Correia, C. F. O.; Nunes, S. C. C.; Pereira, N. A. M.; Ferreira, A. R. F.; Sousa, E. P.; Gomes, C. S. B.; Salvador, J. A. R.; Pais, A. A. C. C.; Pinho e Melo, T. M. V. D. Org. Biomol. Chem. 2015, 13, 9127-9139. doi:10.1039/C5OB01110H

37. Badami, B. V.; Puranik, G. S. Rev. Roum. Chim. 1982, 27, 281-284.

38. Gotthardt, H.; Böhm, F.-R.; Brauer, D. J.; Weisshuhn, C. M.; Wilke, C. J. Chem. Ber. 1988, 121, 95-104. doi:10.1002/cber.19881210115

39. Rao, H. S. P.; Barthasarathy, N. Indian J. Heterocycl. Chem. 2007, 16, 413-414.

40. Li, J.; Wu, F.; Liu, X.; Zou, Y.; Chen, H.; Li, Z.; Zhang, L. Eur. J. Med. Chem. 2017, 140, 20-30. doi:10.1016/j.ejmech.2017.08.047

41. McGowin, A. E.; Jackson, L.; Marshall, L. W.; Tumbull, K. Org. Prep. Proced. Int. 2001, 33, 100-102. doi:10.1080/00304940109356581

42. Wallace, S.; Chin, J. W. Chem. Sci. 2014, 5, 1742-1744. doi:10.1039/C3SC53332H

43. Plougastel, L.; Koniev, O.; Specklin, S.; Decuypere, E.; Créminon, C.; Buisson, D.-A.; Wagner, A.; Kolodych, S.; Taran, F. Chem. Commun. 2014, 50, 9376-9378. doi:10.1039/C4CC03816A

44. Liu, H.; Audisio, D.; Plougastel, L.; Decuypere, E.; Buisson, D.-A.; Koniev, O.; Kolodych, S.; Wagner, A.; Elhabiri, M.; Krzyczmonik, A.; Forsback, S.; Solin, O.; Gouverneur, V.; Taran, F. Angew. Chem., Int. Ed. 2016, 55, 12073-12077. doi:10.1002/anie.201606495

45. Narayanam, M. K.; Liang, Y.; Houk, K. N.; Murphy, J. M. Chem. Sci. 2016, 7, 1257-1261. doi:10.1039/C5SC03259H

46. Liu, F.; Liang, Y.; Houk, K. N. Acc. Chem. Res. 2017, 50, 2297-2308. doi:10.1021/acs.accounts.7b00265

47. Medina, J. M.; McMahon, T. C.; Jiménez-Osés, G.; Houk, K. N.; Garg, N. K. J. Am. Chem. Soc. 2014, 136, 14706-14709. doi:10.1021/ja508635v

48. Lazaris, A. Y. Zh. Org. Khim. 1966, 2, 1322-1323. 
49. Wu, C.; Fang, Y.; Larock, R. C.; Shi, F. Org. Lett. 2010, 12, 2234-2237. doi:10.1021/ol100586r

50. Fang, Y.; Wu, C.; Larock, R. C.; Shi, F. J. Org. Chem. 2011, 76 8840-8851. doi:10.1021/j0201605v

51. Seliverstov, M. Y.; Afanas'ev, O. I.; Sosonyuk, S. E.; Temnov, V. V.; Proskurnina, M. V.; Zefirova, N. S. Dokl. Chem. 2016, 466, 41-44. doi:10.1134/S0012500816020063

52. Li, L.; Wang, H.; Yang, X.; Kong, L.; Wang, F.; Li, X. J. Org. Chem. 2016, 81, 12038-12045. doi:10.1021/acs.joc.6b02356

53. Ikawa, T.; Masuda, S.; Nakajima, H.; Akai, S. J. Org. Chem. 2017, 82, 4242-4253. doi:10.1021/acs.joc.7b00238

54. Huisgen, R.; Gotthardt, H. Chem. Ber. 1968, 101, 1059-1071. doi:10.1002/cber.19681010343

55. Youn, B. H.; Lyu, H. S.; Han, J. H.; Kim, S. H. Bull. Korean Chem. Soc. 1987, 8, 233-235.

56. Hoffmann, R.; Woodward, R. B. J. Am. Chem. Soc. 1965, 87, 2046-2048. doi:10.1021/ja01087a034

57. Sustmann, R. Pure Appl. Chem. 1974, 40, 569-593. doi:10.1351/pac197440040569

58. Houk, K. N.; Sims, J.; Duke, R. E., Jr.; Strozier, R. W.; George, J. K. J. Am. Chem. Soc. 1973, 95, 7287-7301. doi:10.1021/ja00803a017

59. Krauch, C. H.; Kuhls, J.; Piek, H.-J. Tetrahedron Lett. 1966, 7, 4043-4048. doi:10.1016/S0040-4039(00)90284-3

60. Huseya, Y.; Chinone, A.; Ohta, M. Bull. Chem. Soc. Jpn. 1971, 44, 1667-1668. doi:10.1246/bcsj.44.1667

61. Chinone, A.; Huseya, Y.; Ohta, M. Bull. Chem. Soc. Jpn. 1970, 43, 2650. doi:10.1246/bcsj.43.2650

62. George, M. V.; Angadiyavar, C. S. J. Org. Chem. 1971, 36, 1589-1594. doi:10.1021/jo00811a004

63. Gotthardt, H.; Reiter, F. Tetrahedron Lett. 1971, 2749-2752. doi:10.1016/S0040-4039(01)96970-9

64. Gotthardt, H.; Reiter, F. Chem. Ber. 1979, 112, 1206-1225. doi:10.1002/cber.19791120415

65. Märky, M.; Hansen, H.-J.; Schmid, H. Helv. Chim. Acta 1971, 54, 1275-1278. doi:10.1002/hlca.19710540506

66. Sasaki, T.; Kanematsu, K. J. Chem. Soc. C 1971, 2147-2150. doi:10.1039/j39710002147

67. Kishimoto, S.; Noguchi, S.; Masuda, K. Chem. Pharm. Bull. 1976, 24, 3001-3010. doi:10.1248/cpb.24.3001

68. Croce, P. D.; La Rosa, C.; Zecchi, G. J. Chem. Soc., Perkin Trans. 1 1985, 2621-2624. doi:10.1039/p19850002621

69. Ranganathan, D.; Bamezai, S. Synth. Commun. 1985, 15, 259-265. doi:10.1080/00397918508063796

70. Jeon, D. J.; Lee, J. N.; Kim, H. R.; Ryu, E. K. Bull. Korean Chem. Soc. 1998, 19, 725-726.

71. Lee, J. N.; Jeon, D. J.; Kim, Y. M.; Kim, K. M.; Song, H. S. Bull. Korean Chem. Soc. 2000, 21, 761-762.

72. Venkatesan, A. M.; Mansour, T. S.; Abe, T.; Yamamura, I.; Takasaki, T.; Agarwal, A.; Dos Santos, O.; Sum, F.-W.; Lin, Y.-I. Bicyclic 6-alkylidene-penems as ß-lactamases inhibitors. WO Patent WO 2003093279 A1, Nov 13, 2003.

73. Abe, T.; Matsunaga, H.; Mihira, A.; Sato, C.; Ushirogochi, H.; Sato, K.; Takasaki, T.; Venkatesan, A. M.; Mansour, T. S. Process for preparing 6-alkylidene penem derivatives. WO Patent WO 2003093277 A1, Nov 13, 2003.

74. Nikitenko, A. A.; Winkley, M. W.; Zeldis, J.; Kremer, K.; Chan, A. W.-Y.; Strong, H.; Jennings, M.; Jirkovský, I.; Blum, D.; Khafizova, G.; Grosu, G. T.; Venkatesan, A. M. Org. Process Res. Dev. 2006, 10, 712-716. doi:10.1021/op050218b
75. Chang, E.-M.; Wong, F. F.; Chen, T.-H.; Chiang, K.-C.; Yeh, M.-Y. Heterocycles 2006, 68, 1007-1015. doi:10.3987/COM-06-10714

76. Chang, E.-M.; Chen, T.-H.; Wong, F. F.; Chang, E.-C.; Yeh, M.-Y. Synlett 2006, 901-904. doi:10.1055/s-2006-939041

77. Venkatesan, A. M.; Agarwal, A.; Abe, T.; Ushirogochi, H.; Yamamura, I.; Ado, M.; Tsuyoshi, T.; Dos Santos, O.; Gu, Y.; Sum, F.-W.; Li, Z.; Francisco, G.; Lin, Y.-I.; Petersen, P. J.; Yang, Y.; Kumagai, T.; Weiss, W. J.; Shlaes, D. M.; Knox, J. R.; Mansour, T. S. J. Med. Chem. 2006, 49, 4623-4637. doi:10.1021/jm060021p

78. González-Nogal, A. M.; Calle, M.; Cuadrado, P.; Valero, R. Tetrahedron 2007, 63, 224-231. doi:10.1016/j.tet.2006.10.026

79. Browne, D. L.; Helm, M. D.; Plant, A.; Harrity, J. P. A. Angew. Chem., Int. Ed. 2007, 46, 8656-8658. doi:10.1002/anie.200703767

80. Browne, D. L.; Taylor, J. B.; Plant, A.; Harrity, J. P. A. J. Org. Chem. 2009, 74, 396-400. doi:10.1021/jo802240e

81. Browne, D. L.; Vivat, J. F.; Plant, A.; Gomez-Bengoa, E.; Harrity, J. P. A. J. Am. Chem. Soc. 2009, 131, 7762-7769. doi:10.1021/ja902460n

82. Browne, D. L.; Taylor, J. B.; Plant, A.; Harrity, J. P. A. J. Org. Chem. 2010, 75, 984-987. doi:10.1021/jo902514v

83. Carceller Gonzalez, E.; Virgili Bernado, M. $2 \mathrm{H}$-Pyrazolo[4,3- $d$ ]pyrimidin-5-amine derivatives as $\mathrm{H}_{4}$ Histamine receptor antagonists for the treatment of allergic, immunological and inflamatory diseases. WO Patent WO 2010043633 A1, April 22, 2010.

84. Foster, R. S.; Jakobi, H.; Harrity, J. P. A. Tetrahedron Lett. 2011, 52, 1506-1508. doi:10.1016/j.tetlet.2011.01.115

85. Mattes, A.; Helmke, H.; Hillebrand, S.; Peris, G.; Sudau, A.; Rodefeld, L.; Gauger, S.; Benting, J.; Dahmen, P.; Meissner, R.; Wachendorff-Neumann, U.; Hadano, H. Bicyclic pyrimidinyl pyrazoles. WO Patent WO 2011124539 A1, Oct 13, 2011.

86. Dürüst, Y.; Sağırı, A.; Kariuki, B. M.; Knight, D. W. Tetrahedron 2014, 70, 6012-6019. doi:10.1016/j.tet.2014.04.083

87. Chappie, T. A.; Helal, C. J.; Kormos, B. L.; Tuttle, J. B.; Verhoest, P. R. Imidazo-triazine derivatives as PDE10 inhibitors. WO Patent WO 2014177977 A1, Nov 6, 2014.

88. Handa, N. V.; Li, S.; Gerbec, J. A.; Sumitani, N.; Hawker, C. J.; Klinger, D. J. Am. Chem. Soc. 2016, 138, 6400-6403. doi:10.1021/jacs.6b03381

89. Brown, A. W.; Fisher, M.; Tozer, G. M.; Kanthou, C.; Harrity, J. P. A. J. Med. Chem. 2016, 59, 9473-9488. doi:10.1021/acs.jmedchem.6b01128

90. Brown, A. W.; Harrity, J. P. A. J. Org. Chem. 2015, 80, 2467-2472. doi:10.1021/acs.joc.5b00143

91. Brown, A. W.; Harrity, J. P. A. Tetrahedron 2017, 73, 3160-3172. doi:10.1016/j.tet.2017.04.049

92. Comas-Barceló, J.; Foster, R. S.; Fiser, B.; Gomez-Bengoa, E.; Harrity, J. P. A. Chem. - Eur. J. 2015, 21, 3257-3263. doi:10.1002/chem.201406118

93. Totoe, H.; McGowin, A. E.; Turnbull, K. J. Supercrit. Fluids 2000, 18, 131-140. doi:10.1016/S0896-8446(00)00060-7

94. Houk, K. N.; Sims, J.; Watts, C. R.; Luskus, L. J. J. Am. Chem. Soc. 1973, 95, 7301-7315. doi:10.1021/ja00803a018

95. Huppatz, J. L. Aust. J. Chem. 1983, 36, 135-147. doi:10.1071/CH9830135

96. Bernard, A.; Cocco, M. T.; Maccioni, A.; Plumitallo, A. Farmaco, Ed. Sci. 1985, 40, 259-271.

97. Ranganathan, D.; Bamezai, S.; Cun-Heng, H.; Clardy, J. Tetrahedron Lett. 1985, 26, 5739-5742. doi:10.1016/S0040-4039(00)98912-3 
98. Ranganathan, D.; Bamezai, S.; Saini, S. Indian J. Chem., Sect. B 1991, 30, 169-175.

99. Fariňa, F.; Fernández, P.; Fraile, T.; Martín, V. M.; Martín, M. R. Heterocycles 1989, 29, 967-974. doi:10.3987/COM-89-4930

100.Meazza, G.; Zanardi, G.; Piccardi, P. J. Heterocycl. Chem. 1993, 30 365-371. doi:10.1002/jhet.5570300213

101.Chan, D. M.-T.; Kamireddy, B.; Kim, H. B.; Patel, K. M.; Sharpe, P. L.; Casini, M. S.; Xu, M.; Armel, G. R.; Stevenson, T. M. Azolecarboxamide herbicides. WO Patent WO 2004106324 A1, Dec 9, 2004.

102.Rai, G.; Puranik, V. G.; Kalluraya, B.; Hegde, J. C. Synth. Commun. 2006, 36, 1285-1290. doi:10.1080/00397910500518874

103.Plant, A.; Boehmer, J. E.; Black, J.; Sparks, T. D. Isoxazoline derivatives and their use as herbicides. WO Patent WO 2006024820 A1, March 9, 2006.

104.Satheesha Rai, N.; Kalluraya, B. Indian J. Chem., Sect. B 2007, 46, 375-378.

105.Satheesha Rai, N.; Kalluraya, B.; Lingappa, B.; Shenoy, S.; Puranic, V. G. Eur. J. Med. Chem. 2008, 43, 1715-1720. doi:10.1016/j.ejmech.2007.08.002

106.Gao, D.; Zhai, H.; Parvez, M.; Back, T. G. J. Org. Chem. 2008, 73, 8057-8068. doi:10.1021/j0801621d

107.Foster, R. S.; Huang, J.; Vivat, J. F.; Browne, D. L.; Harrity, J. P. A. Org. Biomol. Chem. 2009, 7, 4052-4056. doi:10.1039/b910632d

108.Bisaha, J. J.; Crews, A. D., Jr.; Howard, M. H., Jr.; Sharpe, P. L.; Stevenson, T. M.; Taggi, A. E. Fungicidal bicyclic pyrazoles. WO Patent WO 2009076440 A2, June 18, 2009.

109. Stevenson, T. M.; Marshall, E. A.; Taggi, A. E. Herbicidal pyridazinone derivatives. WO $2009086041 \mathrm{~A} 1$, July 9, 2009.

110.Delaunay, T.; Es-Sayed, M.; Vors, J.-P.; Monteiro, N.; Balme, G. Eur. J. Org. Chem. 2011, 3837-3848. doi:10.1002/ejoc.201100119

111.Chang, E.-C.; Wen, Y.-L.; Chang, C.-H.; Shen, Y.-H.; Wen, S.-B.; Yeh, M.-Y.; Wong, F. F. Tetrahedron 2012, 68, 5920-5924. doi:10.1016/j.tet.2012.04.093

112. Brown, A. W.; Comas-Barceló, J.; Harrity, J. P. A. Chem. - Eur. J. 2017, 23, 5228-5231. doi:10.1002/chem.201701019

113.Shi, J.; Xu, H.; Qiu, D.; He, J.; Li, Y. J. Am. Chem. Soc. 2017, 139, 623-626. doi:10.1021/jacs.6b12161

114.Ikawa, T.; Masuda, S.; Takagi, A.; Akai, S. Chem. Sci. 2016, 7, 5206-5211. doi:10.1039/C6SC00798H

115.Shah, T. K.; Medina, J. M.; Garg, N. K. J. Am. Chem. Soc. 2016, 138, 4948-4954. doi:10.1021/jacs.6b01986

116.Wezeman, T.; Comas-Barceló, J.; Nieger, M.; Harrity, J. P. A.; Bräse, S. Org. Biomol. Chem. 2017, 15, 1575-1579. doi:10.1039/C6OB02518H

117.Lallana, E.; Riguera, R.; Fernandez-Megia, E. Angew. Chem., Int. Ed. 2011, 50, 8794-8804. doi:10.1002/anie.201101019

118.Specklin, S.; Decuypere, E.; Plougastel, L.; Aliani, S.; Taran, F. J. Org. Chem. 2014, 79, 7772-7777. doi:10.1021/jo501420r

119.Decuypere, E.; Specklin, S.; Gabillet, S.; Audisio, D.; Liu, H.; Plougastel, L.; Kolodych, S.; Taran, F. Org. Lett. 2015, 17, 362-365. doi:10.1021/ol503482a

120.Taran, F.; Chaumontet, M.; Kolodych, S.; Rasolofonjatovo Andovola, E. Method for producing pyrazoles, novel pyrazoles and application thereof. WO Patent WO2014122407 A2, Aug 14, 2014.

121.Comas-Barceló, J.; Harrity, J. P. A. Synthesis 2017, 49, 1168-1181. doi:10.1055/s-0036-1588922
122.Adeleke, A. F.; Brown, A. P. N.; Cheng, L.-J.; Mosleh, K. A. M.; Cordier, C. J. Synthesis 2017, 49, 790-801. doi:10.1055/s-0036-1588405

123. Worrell, B. T.; Malik, J. A.; Fokin, V. V. Science 2013, 340, 457-460. doi:10.1126/science. 1229506

124. Conry, R. R. Copper: Inorganic \& Coordination Chemistry. Encyclopedia of Inorganic Chemistry; John Wiley \& Sons, Ltd., 2006.

125.Pallenberg, A. J.; Koenig, K. S.; Barnhart, D. M. Inorg. Chem. 1995, 34, 2833-2840. doi:10.1021/ic00115a009

126. Nitsch, J.; Kleeberg, C.; Fröhlich, R.; Steffen, A. Dalton Trans. 2015, 44, 6944-6960. doi:10.1039/C4DT03706E

127. Comas-Barceló, J.; Blanco-Ania, D.; van den Broek, S. A. M. W.; Niuwland, P. J.; Harrity, J. P. A.; Rutjes, F. P. J. T.

Catal. Sci. Technol. 2016, 6, 4718-4723. doi:10.1039/C5CY02247A

\section{License and Terms}

This is an Open Access article under the terms of the Creative Commons Attribution License

(http://creativecommons.org/licenses/by/4.0), which permits unrestricted use, distribution, and reproduction in any medium, provided the original work is properly cited.

The license is subject to the Beilstein Journal of Organic Chemistry terms and conditions: (https://www.beilstein-journals.org/bjoc)

The definitive version of this article is the electronic one which can be found at: doi:10.3762/bjoc. 14.113 